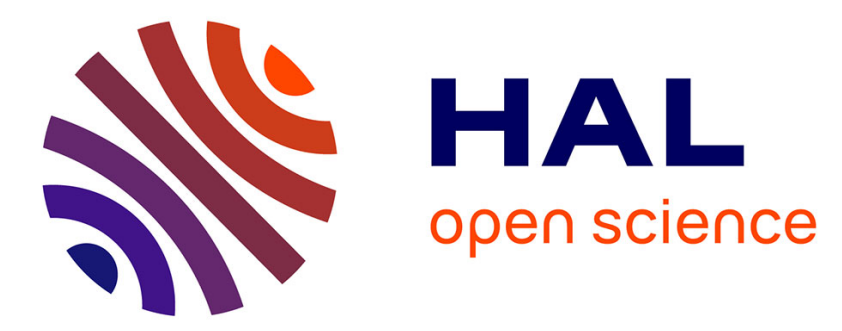

\title{
Study of the non-linear dynamic response of a rotor system with faults and uncertainties
}

Jérôme Didier, Jean-Jacques Sinou, Beatrice Faverjon

\section{To cite this version:}

Jérôme Didier, Jean-Jacques Sinou, Beatrice Faverjon. Study of the non-linear dynamic response of a rotor system with faults and uncertainties. Journal of Sound and Vibration, 2012, 331, pp.671-703. 10.1016/j.jsv.2011.09.001 . hal-00733295

\section{HAL Id: hal-00733295 \\ https://hal.science/hal-00733295}

Submitted on 25 Sep 2012

HAL is a multi-disciplinary open access archive for the deposit and dissemination of scientific research documents, whether they are published or not. The documents may come from teaching and research institutions in France or abroad, or from public or private research centers.
L'archive ouverte pluridisciplinaire HAL, est destinée au dépôt et à la diffusion de documents scientifiques de niveau recherche, publiés ou non, émanant des établissements d'enseignement et de recherche français ou étrangers, des laboratoires publics ou privés. 


\title{
Study of the non-linear dynamic response of a rotor system with faults and uncertainties
}

\author{
Jérôme Didier $^{a}$, Jean-Jacques Sinou $^{a}$ and Béatrice Faverjon ${ }^{b}$ \\ ${ }^{a}$ Laboratoire de Tribologie et Dynamique des Systèmes UMR-CNRS 5513 \\ Ecole Centrale de Lyon, 36 avenue Guy de Collongue \\ 69134 Ecully Cedex, France \\ ${ }^{b}$ Laboratoire de Mécanique des Contacts et des Structures UMR-CNRS 5259 \\ INSA-Lyon, 18-20, rue des Sciences \\ 69621 Villeurbanne Cedex France
}

\begin{abstract}
In this paper, the quantification of uncertainty effects on the variability of the non-linear response in rotor systems with multi-faults (such as unbalance, asymmetric shaft, bow, parallel and angular misalignments) is investigated. To take account of uncertainties in this kind of non linear problem, it is proposed to use the Harmonic Balance Method (HBM) with a Polynomial Chaos Expansion (PCE). The efficiency and robustness of the proposed methodology is demonstrated by comparison with Monte Carlo Simulations (MCS) for different kinds and levels of uncertainties.

Keywords: rotor system, non-linear dynamic, uncertainties.
\end{abstract}

\section{Introduction}

During a phase of preliminary design of dynamic systems such as rotors, many physical parameters can have a significant influence on the vibration response of the system. Also, some features (such as unbalance, misalignment, bow, asymmetric shaft, etc) that can generate linear or non-linear responses need to be taken into account to avoid unexpected vibrational amplitudes and worst design for a rotor system. A state of the art of faults in rotating machinery can be found in [1]:

- Rotor unbalance is one of the most current faults in a rotordynamics problem. This phenomenon is due to the fact that the center of mass of a disc or a shaft element is not on the axis of rotation of the system. It is generally modeled by an eccentric mass which generates a synchronous force.

- Misalignment in coupling is a common fault in rotating machinery, nevertheless this phenomenon remains incompletely understood. It can be noted that there are two cases of misalignment, firstly the parallel misalignment occurs when two shafts have a relative displacement, secondly the angular misalignment occurs when it exists an angular fault between two 
shafts of a rotor system. The presence of a misalignment generates reactions forces and moments in the coupling which are source of vibrations. Consequently the dynamic behavior of the system depends of the characteristics of the coupling. An other distinction have to be done between flexible coupling (such as universal joint) and rigid coupling. Xu and Marangoni [2,3] present a theoretical model and experimental validation for a flexible coupling. Theoretical development for a rigid coupling can also be found in [4]. An other modeling has been developed in $[5,6]$ : Lees presents a simple model of a rigid coupling system with a parallel fault and investigates the influence of misalignment on the dynamic behavior of the system.

- The asymmetry is an other common fault in a rotor problem. Usually this fault can be due to the geometry of the shaft or due to the presence of a crack. Numerous studies have been done to take account of the presence of a crack, and the breathing behavior of the cracked rotor [7,8]. Mathematical development for an asymmetric shaft cross-section has been developed by Oncescu et al. in rotating frame [9] or by Lalanne and Ferraris in fixed frame [10].

- The bow is an initial deformation of the rotor which can be caused by different factors. In [11], Pennacchi presents different sources of bow such as gravity effects on off-line machines or thermal effects. An asymmetric thermal distribution can be localized on an element (local bow) or all parts of the rotor (extended bow). Darpe [12] proposes to investigate the influence of a bow on the dynamic response of the rotor system with other kinds of faults such as unbalance and crack.

- Other kinds of faults exist, such as rubs or ovalization errors [13], but they will not be studied in this paper.

As previously mentioned, all these faults can significantly reduce the life time of a rotating machine. Even if usually the main aim of research studies is to locate individual faults in machine operation, this study proposes to demonstrate the capability to include the treatment of uncertainties in multi-faults in the dynamic analysis of a rotor system, believing that this goal should be useful in the design stage of a machine. For example, some physical parameters may vary in an uncertain way during the manufacturing monitoring or the use process (like flight or others) and so the response may change also in one uncertain way. So, the novelty of the present paper is to propose a formulation of dynamical systems such as rotor with multi-faults, which includes uncertainties in physical parameters. Thus, the goal is to allow an estimate of the overall nonlinear dynamic responses generated by these considerations. Considering this, there are different methods to take account of uncertainties in this kind of problem such as perturbation method, Monte Carlo simulations or Polynomial Chaos Expansion. The perturbation and Neumann methods based respectively on the expansion of random quantities into Taylor series [14] and Neumann series [15, 16], provide acceptable results for small random fluctuations but are not adapted for solving a dynamic problem close to resonance frequency. The Monte Carlo simulations (MCS) is well adapted to include uncertainties in a deterministic finite element model by generating samples of the random parameter. Nevertheless MCS have a high computational cost due to the fact that a high number of samples is necessary to have convergence of this method. The Polynomial Chaos Expansion (PCE) [17] is a method based on the representation of the stochastic processes and variables in a set of orthogonal bases of random variables. This method has 
shown to be a successful approach to solve uncertainty quantification problems that is why it is used in the present study.

So the objective of the present study is to propose a methodology to undertake the effects of uncertainties on the non-linear dynamic response of a rotating machinery with multi-faults. The present paper is organized as follows: in a first part, a brief description of the rotor system is given. Then the different faults in rotating machine studied in this paper are presented. As previously explained, the novelty in the attempted work appears in the formulation of such rotor system model that includes the uncertainties in physical parameters. So the second part of the paper presents the methodology of the Polynomial Chaos Expansion (PCE) with the Harmonic Balance Method in order to estimate evolutions of the $n \times$ amplitudes and the global non-linear responses of a rotor system with multi-faults and uncertainties. Then, the expansions of the random quantities for all the models of faults of the rotor system are presented. Finally the efficiency of the method is demonstrated through various numerical simulations and a comparison of the results obtained via Polynomial Chaos Expansion (PCE) and via Monte Carlo Simulation (MCS).

\section{Rotor system}

\subsection{General equation of a rotor system}

The system under study is illustrated in Figure 1. The rotor consists of a shaft with two discs. The shaft is discretized into 10 Euler beam finite elements with four degrees of freedom at each node, with an asymmetrical section on the elements 3,4 and 5 and a constant circular section on the other elements. Two bearing supports are added at each end of the rotor. All the values of the parameters are given in Table 1.

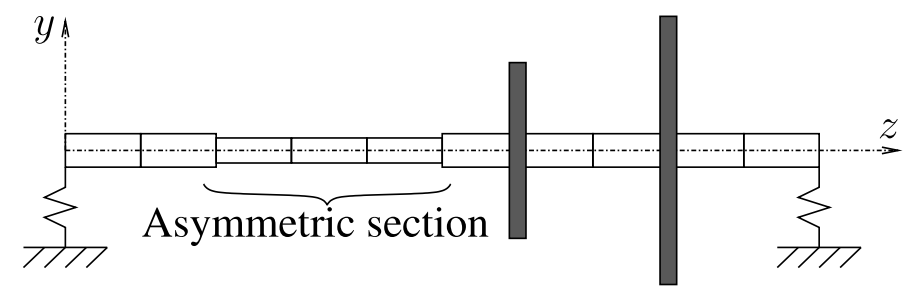

Figure 1: Rotor system

After assembling the shaft elements, the discs and the bearing supports, the equation of motion of the complete rotor system is given by:

$$
\mathbf{M} \ddot{\mathbf{x}}+(\mathbf{C}+\omega \mathbf{G}) \dot{\mathbf{x}}+\left(\mathbf{K}_{s}+\mathbf{K}_{b}\right) \mathbf{x}=\mathbf{f}
$$

where $\mathbf{M}$ and $\mathbf{G}$ are the mass and gyroscopic matrices of the system. $\mathbf{K}_{s}$ and $\mathbf{K}_{b}$ are respectively the stiffness matrices of the asymmetric shaft and the bearings. $\mathbf{C}$ is the damping matrix of the shaft. $\mathbf{f}$ are the forces applied on the system including gravitational forces, unbalance forces and forces due to faults. $\ddot{\mathrm{x}}, \dot{\mathrm{x}}$ and $\mathrm{x}$ are the acceleration, the velocity and the displacement of the degrees of freedom 


\begin{tabular}{ll}
\hline Parameters & dimension \\
\hline Length of shaft $L$ & $1 \mathrm{~m}$ \\
Diameter of shaft & $0.04 \mathrm{~m}$ \\
Position of disc 1 & $0.6 \mathrm{~m}$ \\
Position of disc 2 & $0.8 \mathrm{~m}$ \\
Outer diameter of disc 1 & $0.2 \mathrm{~m}$ \\
Outer diameter of disc 2 & $0.4 \mathrm{~m}$ \\
Inner diameter of discs 1 and 2 & $0.04 \mathrm{~m}$ \\
Thickness of discs 1 and 2 & $0.02 \mathrm{~m}$ \\
Young modulus of elasticity $E$ & $2.1 \times 10^{11} \mathrm{Nm}^{2}$ \\
Shear modulus $G$ & $8.0 \times 10^{10} \mathrm{Nm}^{2}$ \\
Poisson ratio $\nu$ & 0.3 \\
Density $\rho$ & $7800 \mathrm{~kg} \mathrm{~m}^{-3}$ \\
Mass unbalance & $0.05 \mathrm{~g}$ \\
Eccentricity of the mass unbalance & $0.01 \mathrm{~m}$ \\
Damping coefficient $\alpha_{d}$ & $2.5 \times 10^{-5}$ \\
Damping coefficient $\beta_{d}$ & 2.8 \\
Depth of the asymmetry $h$ & $0.002 \mathrm{~m}^{-5}$ \\
Phase of the asymmetry $\psi_{a}$ & $0^{\circ}$ \\
Phase of the bow $\psi_{b}$ & $0^{\circ}$ \\
Axial stiffness of bolt $k$ & $5.2 \times 10^{3} \mathrm{Nm}^{-1}$ \\
Transversal stiffness of bolt $k_{b}$ & $13 \times 10^{3} \mathrm{Nm}^{-1}$ \\
\hline
\end{tabular}

Table 1: Model parameters

of the rotor system. $\omega$ is the rotating speed of the shaft. It should be noted that all these matrices are expressed in the fixed frame, their construction will be detailed in the following parts of the paper.

\subsection{Faults in rotating machinery}

In this paper it has been chosen to investigate the most current faults in a rotor system. Four kinds of faults are studied here: an unbalance rotor, an asymmetric shaft cross section, a bended shaft and a misalignment fault.

In this section the rotating and fixed frames are respectively noted $R(X, Y, z)$ and $R_{0}(x, y, z)$, and are linked by the following basis change matrix defined as

$$
P_{R \rightarrow R_{0}}=\left[\begin{array}{ccc}
\cos (\omega t) & -\sin (\omega t) & 0 \\
\sin (\omega t) & \cos (\omega t) & 0 \\
0 & 0 & 1
\end{array}\right]
$$




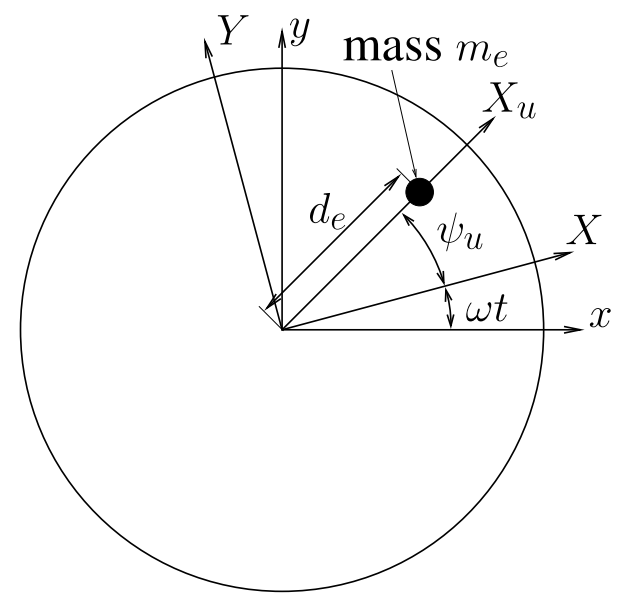

Figure 2: Unbalance disc

\subsubsection{Unbalance}

The unbalance is one of the most common fault in a rotor problem. It appears when the center of mass of a shaft element or a disc is not on the axis of rotation of the system. So this fault is usually modeled by an eccentric mass on a disc or on the shaft, as illustrated in Figure 2.

For the degrees of freedom $\left[\begin{array}{llll}v & w & \theta & \psi\end{array}\right]^{T}$ of a disc, the eccentric mass can be modeled by the following forces system

$$
\mathbf{f}^{d}=\left[\begin{array}{llll}
m_{e} d_{e} \omega^{2} \cos \left(\omega t+\psi_{u}\right) & m_{e} d_{e} \omega^{2} \sin \left(\omega t+\psi_{u}\right) & 0 & 0
\end{array}\right]^{T}
$$

where $m_{e}$ and $d_{e}$ are respectively the mass unbalance and the eccentricity. $\psi_{u}$ defines the initial angular position in relation to z-axis.

\subsubsection{Rotor asymmetry}

The shaft cross-section of the elements 3, 4 and 5 is asymmetric. The geometry of the section is given in Figure 3.

Consequently the asymmetric section has different principal moments of inertia in rotating frame $\left(X_{a}, Y_{a}, z\right)$ which are given by the following expressions

$$
\begin{aligned}
I_{X} & =\iint Y^{2} d S \\
I_{Y} & =\iint X^{2} d S
\end{aligned}
$$

After calculations, we obtain

$$
\begin{aligned}
& I_{X}=\frac{R^{4}}{2}\left(\alpha-\frac{\sin (2 \alpha)}{2}\right)+\sqrt{2 h R-h^{2}}(R-h)^{3} \\
& I_{Y}=\frac{R^{4}}{2}\left(\alpha+\frac{\sin (2 \alpha)}{2}\right)+\frac{\left(2 h R-h^{2}\right)^{\frac{3}{2}}(R-h)}{3}
\end{aligned}
$$




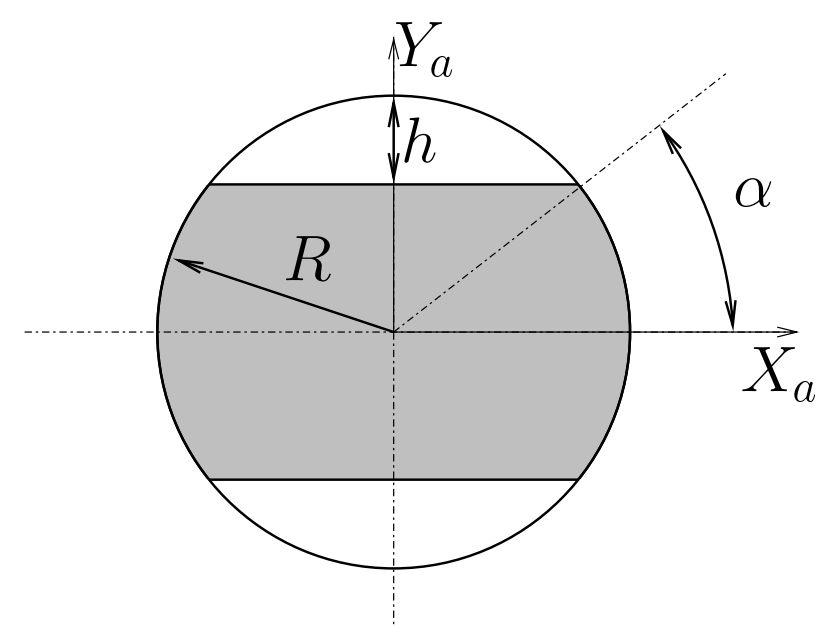

Figure 3: Asymmetric shaft cross-section

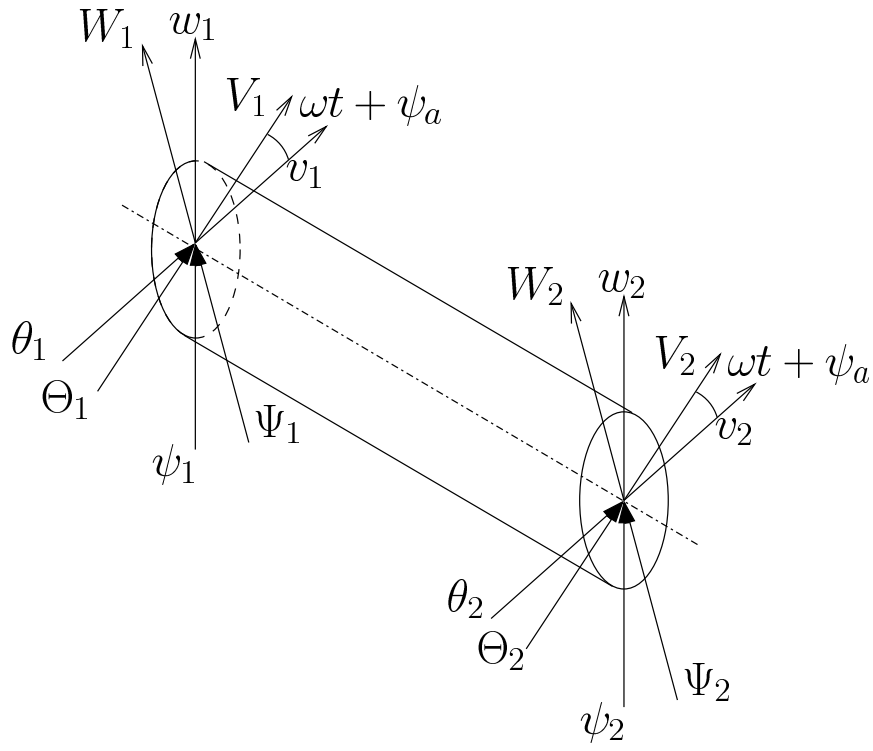

Figure 4: Coordinates in fixed frame and rotating frame

where $\alpha, R$ and $h$ define the angle, the radius and the depth of the asymmetric section as illustrated in Figure 3.

In the following part of this section, the stiffness and mass matrices due to this rotor asymmetry will be calculated by using expressions of the strain and kinetic energies.

Strain energy The nodal displacement vector is defined as $\delta_{f i x}=\left[\begin{array}{llllllll}v_{1} & w_{1} & \theta_{1} & \psi_{1} & v_{2} & w_{2} & \theta_{2} & \psi_{2}\end{array}\right]^{T}$ in

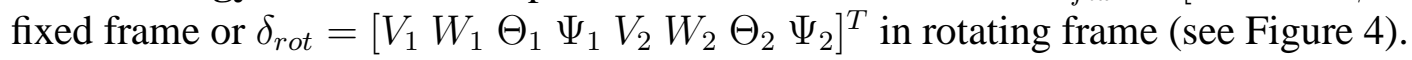


The strain energy of the shaft element is defined as

$$
U=\frac{1}{2} \int_{0}^{l} E I_{X}\left(V^{\prime \prime}\right)^{2}+E I_{Y}\left(W^{\prime \prime}\right)^{2} d z
$$

where $V$ and $W$ are the displacements in rotating frame. $l$ is the length of the element. $E$ is the Young modulus. Shear effects are neglected.

By using the following relations

$$
\left\{\begin{array}{l}
V=\cos \left(\omega t+\psi_{a}\right) v+\sin \left(\omega t+\psi_{a}\right) w \\
W=-\sin \left(\omega t+\psi_{a}\right) v+\cos \left(\omega t+\psi_{a}\right) w
\end{array}\right.
$$

with $v$ and $w$ the displacements in fixed frame, the strain energy can be rewritten as follows

$$
\begin{aligned}
U & =\frac{1}{2} \int_{0}^{l} E I_{m}\left(v^{\prime \prime 2}+w^{\prime \prime 2}\right) d z+\frac{1}{2} \int_{0}^{l} E I_{d}\left(v^{\prime \prime 2}-w^{\prime \prime 2}\right) \cos \left(2 \omega t+2 \psi_{a}\right) d z \\
& +\int_{0}^{l} E I_{d} v^{\prime \prime} w^{\prime \prime} \sin \left(2 \omega t+2 \psi_{a}\right) d z
\end{aligned}
$$

where $I_{m}$ and $I_{d}$ are the mean and deviatoric moments of the area and are equal to $I_{m}=\frac{I_{X}+I_{Y}}{2}$ and $I_{d}=\frac{I_{X}-I_{Y}}{2}$.

This energy can be calculated by using classical shape functions [10] defined as

$$
\left(\begin{array}{c}
v \\
w
\end{array}\right)=\left(\begin{array}{c}
N_{1} v_{1}+N_{2} v_{2}+N_{3} \psi_{1}+N_{4} \psi_{2} \\
N_{1} w_{1}+N_{2} w_{2}-N_{3} \theta_{1}-N_{4} \theta_{2}
\end{array}\right)
$$

where $N_{1}=1-\frac{3 z^{2}}{l^{2}}+\frac{2 z^{3}}{l^{3}}, N_{2}=\frac{3 z^{2}}{l^{2}}-\frac{2 z^{3}}{l^{3}}, N_{3}=-z+\frac{2 z^{2}}{l}-\frac{z^{3}}{l^{2}}$ and $N_{4}=\frac{z^{2}}{l}-\frac{z^{3}}{l^{2}}$.

After calculations, the strain energy can be expressed as follows

$$
U=\frac{1}{2} \delta_{f i x}^{T} \mathbf{K}^{e} \delta_{f i x}
$$

where the stiffness matrix $\mathbf{K}^{e}$ is defined in fixed frame as

$$
\mathbf{K}^{e}=\mathbf{K}_{0}^{e}+\mathbf{K}_{c}^{e} \cos \left(2 \omega t+2 \psi_{a}\right)+\mathbf{K}_{s}^{e} \sin \left(2 \omega t+2 \psi_{a}\right)
$$

The expressions of $\mathbf{K}_{0}^{e}, \mathbf{K}_{c}^{e}$ and $\mathbf{K}_{s}^{e}$ are given by

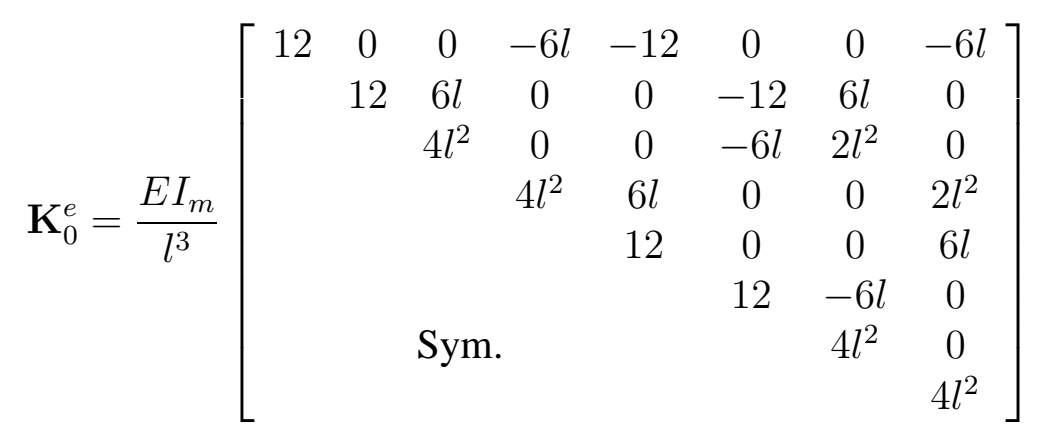




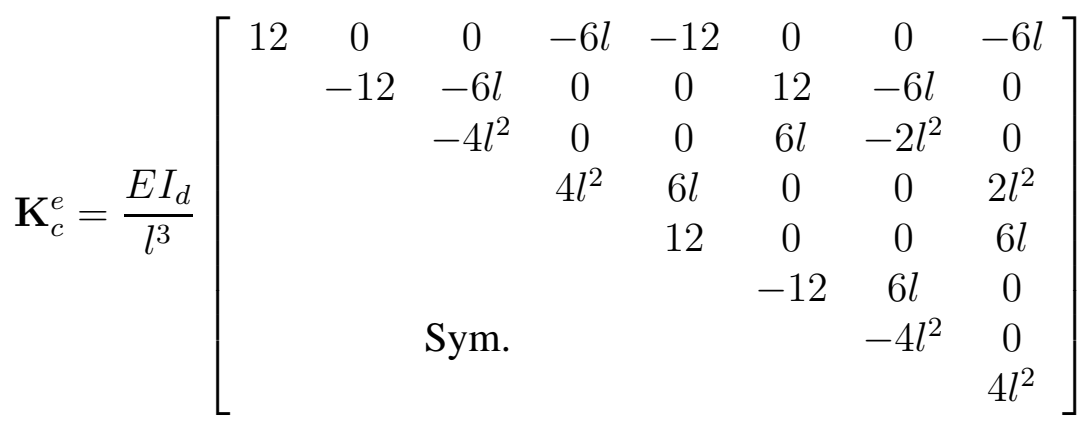

$$
\begin{aligned}
& \mathbf{K}_{s}^{e}=\frac{E I_{d}}{l^{3}}\left[\begin{array}{cccccccc}
0 & 12 & 6 l & 0 & 0 & -12 & 6 l & 0 \\
& 0 & 0 & -6 l & -12 & 0 & 0 & -6 l \\
& & 0 & -4 l^{2} & -6 l & 0 & 0 & -2 l^{2} \\
& & & 0 & 0 & 6 l & -2 l^{2} & 0 \\
& & & 0 & 12 & -6 l & 0 \\
& & & & 0 & 0 & 6 l \\
& & & & & & 0 & -4 l^{2} \\
& & & & & & & 0
\end{array}\right]
\end{aligned}
$$

where $l$ is the length of the shaft element.

Kinetic energy The kinetic energy of the shaft element is defined as

$$
T=\frac{1}{2} \rho S \int_{0}^{l}\left(\dot{v}^{2}+\dot{w}^{2}\right) d z+\frac{1}{2} \rho \int_{0}^{l}\left(I_{X} \omega_{X}^{2}+I_{Y} \omega_{Y}^{2}+2 I_{m} \omega_{Z}^{2}\right) d z
$$

where $\omega_{X}, \omega_{Y}$ and $\omega$ are the angular velocities of the shaft element and can be approximated as [10]

$$
\left\{\begin{array}{l}
\omega_{X}=\dot{\theta} \sin \left(\omega t+\psi_{a}\right)-\dot{\psi} \sin \left(\omega t+\psi_{a}\right) \\
\omega_{Y}=\dot{\psi} \cos \left(\omega t+\psi_{a}\right)+\dot{\theta} \sin \left(\omega t+\psi_{a}\right) \\
\omega_{Z}=\omega+\dot{\psi} \theta
\end{array}\right.
$$

After calculations, the kinetic energy can be rewritten as follows

$$
\begin{aligned}
T & =\frac{1}{2} \rho S \int_{0}^{l}\left(\dot{v}^{2}+\dot{w}^{2}\right) d z+\frac{1}{2} \rho I_{m} \int_{0}^{l}\left(\dot{\theta}^{2}+\dot{\psi}^{2}\right) d z \\
& +\frac{1}{2} \rho I_{d} \int_{0}^{l}\left(\dot{\theta}^{2}+\dot{\psi}^{2}\right) \cos \left(2 \omega t+2 \psi_{a}\right) d z+\frac{1}{2} \rho I_{d} \int_{0}^{l} \dot{\psi} \theta \sin \left(2 \omega t+2 \psi_{a}\right) d z \\
& +\rho I_{m} \omega \int_{0}^{l} \dot{\psi} \theta d z
\end{aligned}
$$

By using the shape functions and after integration of the previous equation, it leads to express the gyroscopic matrix and the mass matrices. 
The gyroscopic matrix is defined as

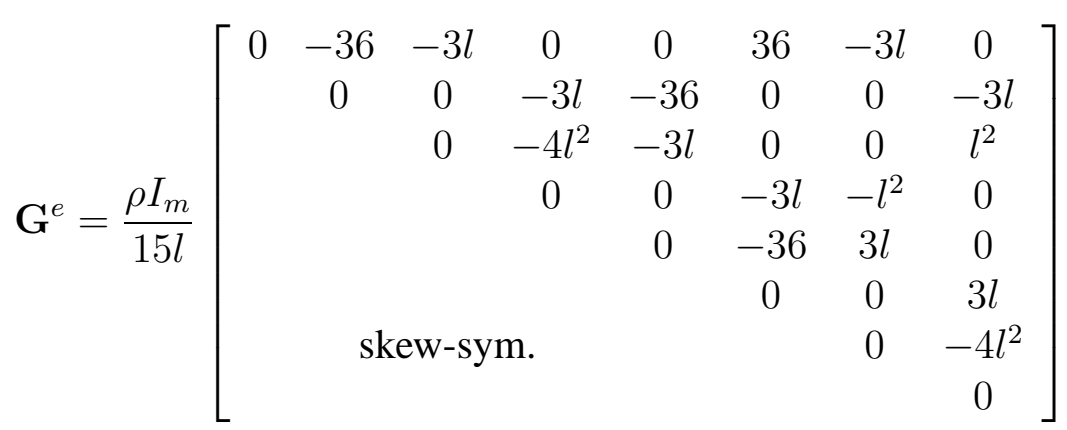

The elementary mass matrix is defined in fixed frame as

$$
\mathbf{M}^{e}=\mathbf{M t}_{0}^{e}+\mathbf{M r}_{0}^{e}+\mathbf{M}_{c}^{e} \cos \left(2 \omega t+2 \psi_{a}\right)+\mathbf{M}_{s}^{e} \sin \left(2 \omega t+2 \psi_{a}\right)
$$

The expression $\mathbf{M t}_{0}^{e}, \mathbf{M r}_{0}^{e}, \mathbf{M}_{c}^{e}$ and $\mathbf{M}_{s}^{e}$ are given by

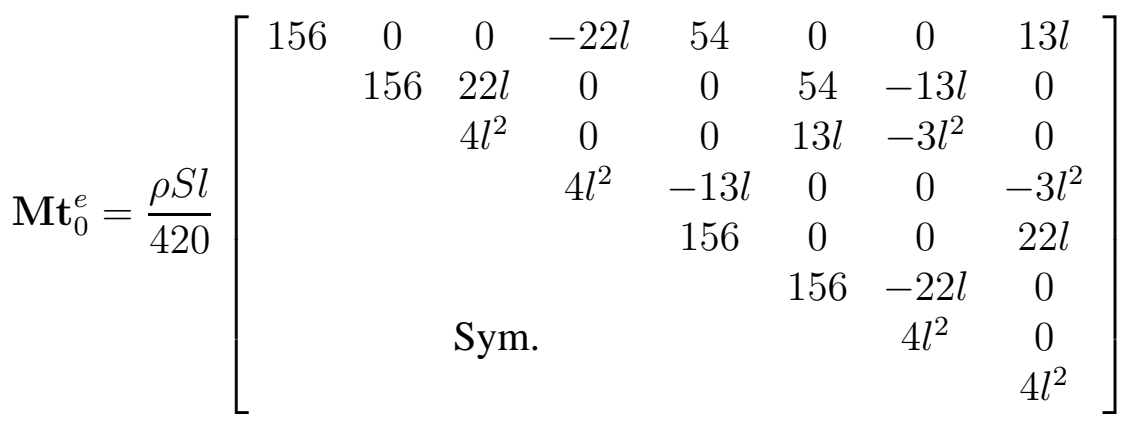

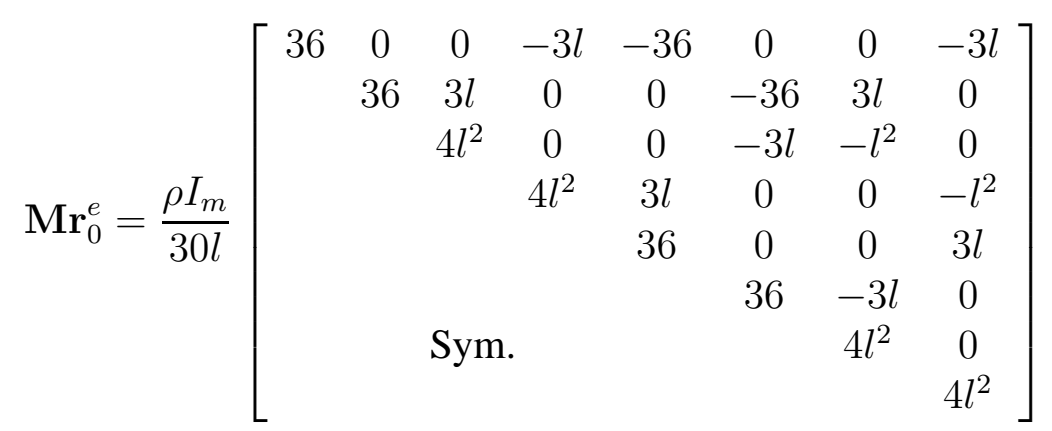

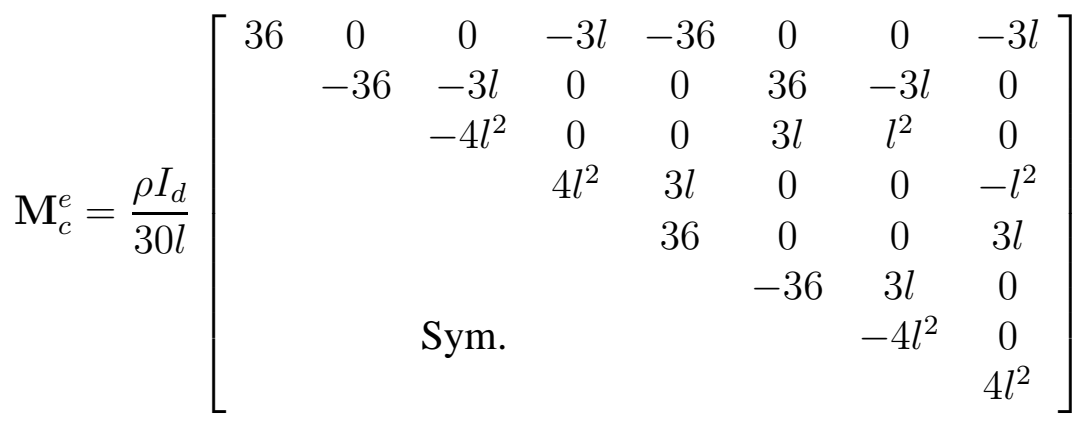




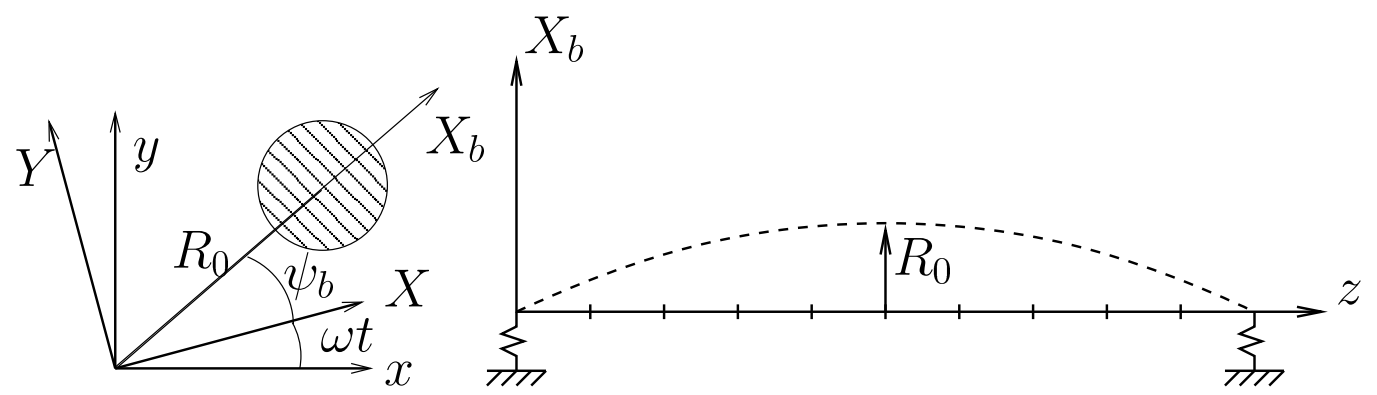

Figure 5: Coordinate system of the bow

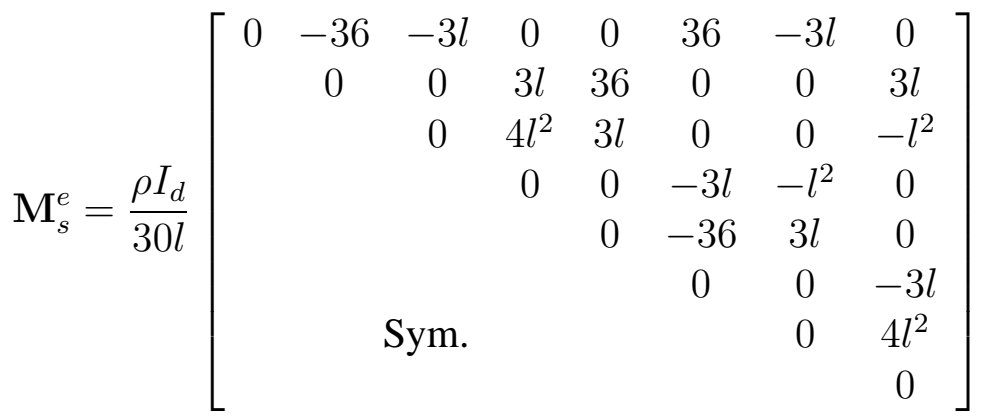

where $\rho$ is the density.

\subsubsection{Rotor bow}

In this section, the study of the rotor in the presence of an initial deformation of the shaft is proposed. This deformation that is illustrated in Figure 5, is defined in fixed frame as $\mathbf{x}_{\mathbf{0}}$, so the equation of the dynamics problem is given by

$$
\mathbf{M}_{r} \ddot{\mathbf{x}}_{r}+\mathbf{D}_{r} \dot{\mathbf{x}}_{r}+\mathbf{K}_{r}\left(\mathbf{x}_{r}-\mathbf{x}_{\mathbf{0} r}\right)=\mathbf{f}_{r}
$$

where $\mathbf{M}_{r}, \mathbf{D}_{r}$ and $\mathbf{K}_{r}$ are respectively the mass, the damping and the stiffness matrices expressed in the rotating frame. The subscript $r$ denotes the fact that the quantity is expressed in the rotating frame. For the reader comprehension, the deformation $\mathbf{x}_{\mathbf{0}_{r}}$ in rotating frame $R_{b}\left(X_{b}, Y_{b}, z\right)$ is defined as

$$
\mathbf{x}_{\mathbf{0} r}=\left[\begin{array}{lllll}
\mathbf{x}_{\mathbf{0}}{ }^{(1)} & \ldots & \mathbf{x}_{\mathbf{0}}{ }^{(k)} & \ldots & \mathbf{x}_{\mathbf{0}}{ }_{r}^{(n)}
\end{array}\right]^{T}
$$

where $\mathbf{x}_{\mathbf{0}}{ }^{(k)}$ are the displacements of the $k^{t h}$ node in $R_{b}$.

Consequently the initial deformation can be modeled as a force defined by

$$
\mathbf{f}_{r}^{b}=\mathbf{K}_{r} \mathbf{x}_{\mathbf{0} r}
$$

In the present paper, it has been chosen to study a bowed rotor which has the geometric properties 


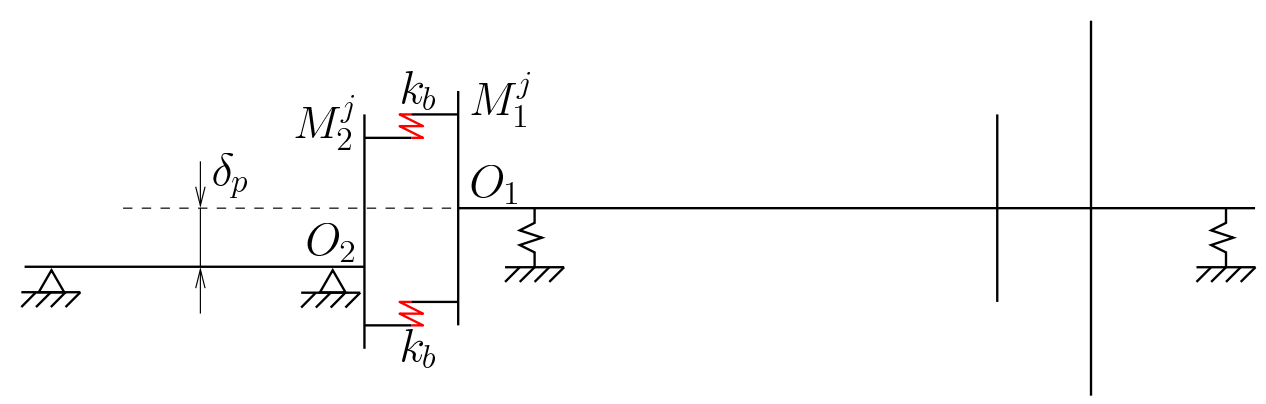

Figure 6: Model of parallel misalignment

presented in Figure 5. Consequently

$$
\begin{aligned}
\mathbf{x}_{\mathbf{0}}{ }_{r}^{(k)} & =\left[\begin{array}{llll}
V^{(k)} & 0 & 0 & \Phi^{(k)}
\end{array}\right]^{T} \\
& =\left[\begin{array}{lllll}
R_{0} \sin \left(\frac{k-1}{n-1} \pi\right) & 0 & 0 & \frac{R_{0} \pi}{L} \cos \left(\frac{k-1}{n-1} \pi\right)
\end{array}\right]^{T}
\end{aligned}
$$

where $R_{0}$ is the maximum of amplitude of the bow.

Finally the force $\mathbf{f}_{r}^{b}$ is expressed in the fixed frame by using the basis change matrices between the rotating frames $R_{b}\left(X_{b}, Y_{b}, z\right)$ and $R_{0}(x, y, z)$. So after calculations this force $\mathbf{f}_{r}^{b}$ can be rewritten as synchronous rotating forces defined in the fixed frame by

$$
\mathbf{f}^{b}=\mathbf{f}_{c}^{b} \cos \left(\omega t+\Psi_{b}\right)+\mathbf{f}_{s}^{b} \sin \left(\omega t+\Psi_{b}\right)
$$

\subsubsection{Misalignment}

Parallel misalignment In this part, the modeling proposed by Lees in [6] is used. The coupling of the two parallel rotors of relative displacement $\delta_{p}$, is composed of $N$ bolts. On the first shaft the bolts are distributed on a circle of radius $r$, on the second rotor the bolts are distributed on a same circle with an offset $\delta_{p}$ from the axis of rotation. This configuration is illustrated on Figure 6.

In the present study it will be considered that the two rotors are acting at the same rotation speed. So the torsional deflection is not taking into account. The relative displacement is only considered in the vertical direction. The initial configuration of the coupling composed of $N=3$ bolts is defined in Figure 7.

Due to the geometry of the coupling the extremities of each bolt have different trajectories. Consequently during a rotation of the coupling system, the energy stored in each bolt will evolve. This energy can be calculated with the trajectories of the bolt extremities and depends on the stiffness $k_{b}$ of bolt in $x$-direction and the $y$-direction.

For the first disc, the trajectory $\overrightarrow{O_{1} M_{1}^{j}}$ of the $j^{\text {th }}$ bolt (for $\mathrm{j}=1,2$ or 3 ) is defined in rotating frame as

$$
\overrightarrow{O_{1} M_{1}^{j}}=\left[\begin{array}{c}
-r \sin \left(\phi_{j}\right) \\
r \cos \left(\phi_{j}\right) \\
0
\end{array}\right]_{R}
$$




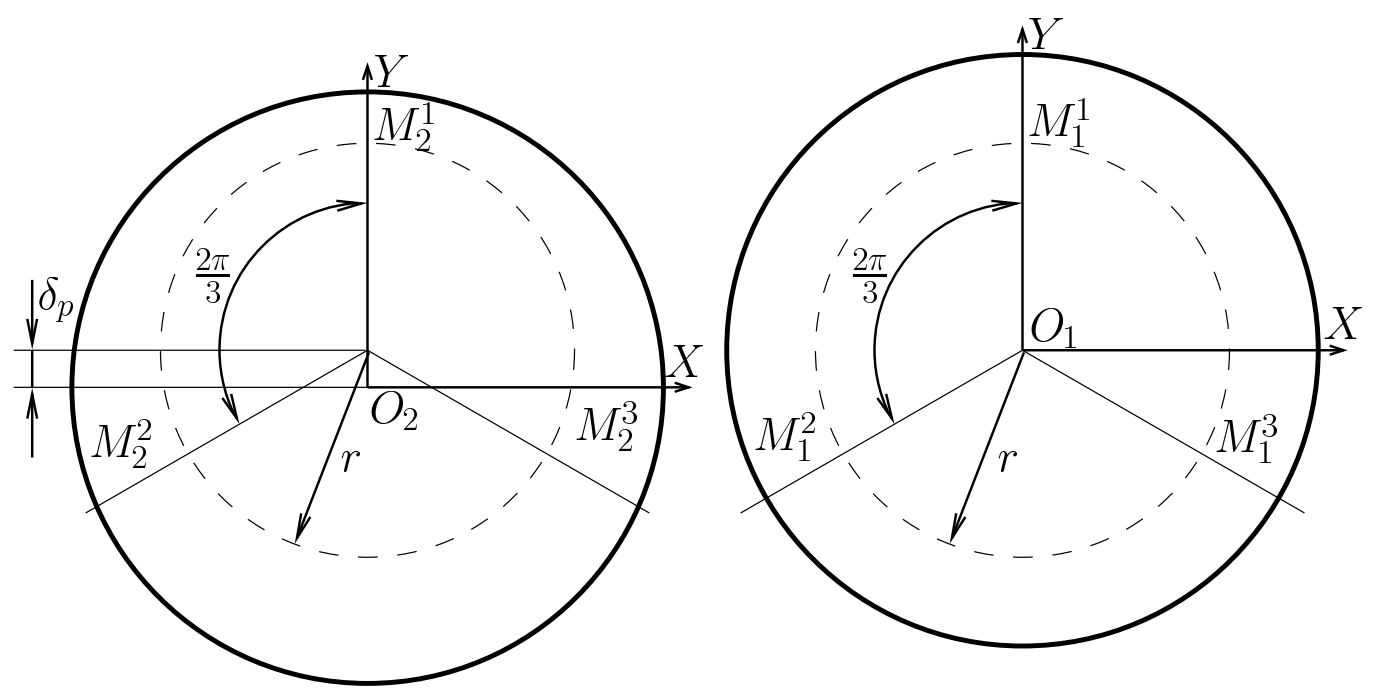

Figure 7: Initial positions of bolts

where $\phi_{j}=(j-1) \frac{2 \pi}{N}$ is the angle between two consecutive bolts ( $N$ defines the number of bolts).

By introducing the basis change and the degrees of freedom $\left[\begin{array}{lllll}v_{c} & w_{c} & \theta_{c} & \psi_{c}\end{array}\right]^{T}$ of the shaft extremity, these trajectories can be rewritten as

$$
\overrightarrow{O M_{1}^{j}}=\left[\begin{array}{c}
v_{c} \\
w_{c} \\
0
\end{array}\right]_{R_{0}}+\left[\begin{array}{c}
-r \sin \left(\omega t+\phi_{j}\right) \\
r \cos \left(\omega t+\phi_{j}\right) \\
r \theta \cos \left(\omega t+\phi_{j}\right)+r \psi \sin \left(\omega t+\phi_{j}\right)
\end{array}\right]_{R_{0}}
$$

For the second disc, the trajectory of the $j^{\text {th }}$ bolt (for $\mathrm{j}=1,2$ or 3 ) is defined in rotating frame as

$$
\overrightarrow{O_{2} M_{2}^{j}}=\left[\begin{array}{c}
-r \sin \left(\phi_{j}\right) \\
\delta_{p}+r \cos \left(\phi_{j}\right) \\
0
\end{array}\right]_{R}
$$

or in fixed frame as

$$
\overrightarrow{O_{2} M_{2}^{j}}=P_{R \rightarrow R_{0}}\left[\begin{array}{c}
-r \sin \left(\phi_{j}\right) \\
\delta_{p}+r \cos \left(\phi_{j}\right) \\
0
\end{array}\right]_{R}=\left[\begin{array}{c}
-r \sin \left(\omega t+\phi_{j}\right)-\delta_{p} \sin (\omega t) \\
r \cos \left(\omega t+\phi_{j}\right)+\delta_{p} \cos (\omega t) \\
0
\end{array}\right]_{R_{0}}
$$

and

$$
\overrightarrow{O M_{2}^{j}}=\left[\begin{array}{c}
-r \sin \left(\omega t+\phi_{j}\right)-\delta_{p} \sin (\omega t) \\
r \cos \left(\omega t+\phi_{j}\right)-\delta_{p}(1-\cos (\omega t)) \\
0
\end{array}\right]_{R_{0}}
$$




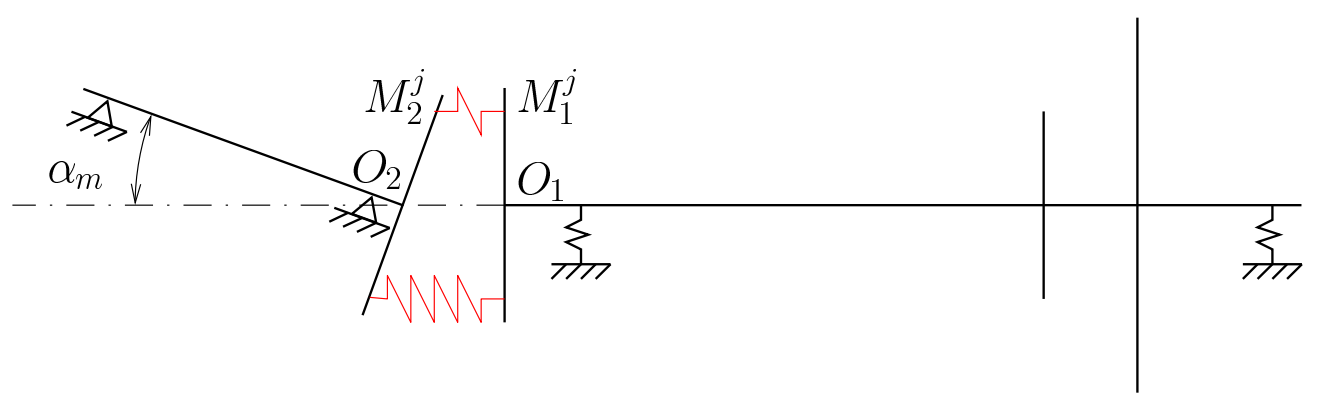

Figure 8: Model of angular misalignment

By using the previous equations, the strain energy can be expressed as

$$
\begin{aligned}
U_{m 1} & =\frac{1}{2} k_{b} \sum_{j=1}^{N}\left[\left(\overrightarrow{O M_{1}^{j}}-\overrightarrow{O M_{2}^{j}}\right) \cdot \vec{x}\right]^{2}+\left[\left(\overrightarrow{O M_{1}^{j}}-\overrightarrow{O M_{2}^{j}}\right) \cdot \vec{y}\right]^{2} \\
& =\frac{1}{2} k_{b} \sum_{j=1}^{N}\left(v_{c}+\delta_{p} \sin (\omega t)\right)^{2}+\left(w_{c}+\delta_{p}(1-\cos (\omega t))\right)^{2}
\end{aligned}
$$

After summation on each bolt of the same properties, the total strain energy due to the parallel misalignment can be rewritten as

$$
U_{m 1}=\frac{1}{2} N k_{b}\left[\left(v_{c}+\delta_{p} \sin (\omega t)\right)^{2}+\left(w_{c}+\delta_{p}(1-\cos (\omega t))\right)^{2}\right]
$$

Finally the Lagrange's equation is applied to the degree of freedom $\left[\begin{array}{llll}v_{c} & w_{c} & \theta_{c} & \psi_{c}\end{array}\right]^{T}$ of the shaft extremity. Consequently after calculations, it results that the presence of a coupling with a parallel misalignment leads to add to the previous system: a stiffness component due to the coupling which is defined as

$$
\mathbf{K}^{m_{1}}=N k_{b}\left[\begin{array}{cccc}
1 & & & \\
& 1 & & \\
& & 0 & \\
& & & 0
\end{array}\right]
$$

and a synchronous force due to the misalignment

$$
\mathbf{f}^{m_{1}}=N k_{b} \delta_{p}[\sin (\omega t) \quad 1-\cos (\omega t) \quad 0 \quad 0]^{T}
$$

Angular misalignment The proposed model of angular misalignment is illustrated in Figure 8. It is composed of two discs with an angular fault $\alpha_{m}$ and $N=3$ bolts. The first bolt has a stiffness $k+k^{\prime}$ in z-direction and the others bolts have a stiffness $k$.

In the following part, an approach similar to these developed in the case of a parallel misalignment is proposed. The position $\overrightarrow{O M_{1}^{j}}$ of the left extremity of the $j^{\text {th }}$ bolt (with $\mathrm{j}=1,2$ or 3 ) is identical to 


\begin{tabular}{lll}
\hline$j$ & $k_{j}$ & $\phi_{j}$ \\
\hline 1 & $k+k^{\prime}$ & 0 \\
2 & $k$ & $2 \pi / 3$ \\
3 & $k$ & $4 \pi / 3$ \\
\hline
\end{tabular}

Table 2: Coupling parameters

the development given in Equation (32). Then, the second shaft have an angular fault $\alpha_{m}$ in $x$-axis as illustrated in Figure 8. Consequently the position $\overrightarrow{\mathrm{O}_{2} M_{2}^{j}}$ can be defined as

$$
\overrightarrow{O_{2} M_{2}^{j}}=r\left[\begin{array}{c}
-\sin \left(\omega t+\phi_{j}\right) \\
\cos \left(\omega t+\phi_{j}\right) \cos \left(\alpha_{m}\right) \\
\cos \left(\omega t+\phi_{j}\right) \sin \left(\alpha_{m}\right)
\end{array}\right]_{R_{0}}
$$

Assuming that the angular $\alpha_{m}$ is small, the following approximations $\cos \left(\alpha_{m}\right) \simeq 1$ and $\sin \left(\alpha_{m}\right) \simeq \alpha_{m}$ can be done. So the position $\overrightarrow{O_{2} M_{2}^{j}}$ can be rewritten as

$$
\overrightarrow{O_{2} M_{2}^{j}}=r\left[\begin{array}{c}
-\sin \left(\omega t+\phi_{j}\right) \\
\cos \left(\omega t+\phi_{j}\right) \\
\alpha_{m} \cos \left(\omega t+\phi_{j}\right)
\end{array}\right]_{R_{0}}
$$

Consequently the strain energy of one bolt can be rewritten as

$$
\begin{aligned}
U_{m 2}^{j} & =\frac{1}{2} k_{j}\left[\left(\overrightarrow{O M_{1}^{j}}-\overrightarrow{O M_{2}^{j}}\right) \cdot \vec{z}\right]^{2} \\
& =\frac{1}{2} k_{j} r^{2}\left[\left(\theta-\alpha_{m}\right) \cos \left(\omega t+\phi_{j}\right)+\psi \sin \left(\omega t+\phi_{j}\right)\right]^{2}
\end{aligned}
$$

So the strain energy for all bolts is defined as

$$
U_{m 2}=\sum_{i=j}^{N} U_{j}
$$

which after calculations gives the following expression

$$
\begin{aligned}
U_{m 2} & =\frac{1}{4}\left(3 k+k^{\prime}\right) r^{2}\left[\left(\alpha_{m}-\theta\right)^{2}+\psi^{2}\right] \\
& +\frac{1}{4} k^{\prime} r^{2}\left[\left(\left(\alpha_{m}-\theta\right)^{2}+\psi^{2}\right) \cos (2 \omega t)-2 \psi\left(\alpha_{m}-\theta\right) \sin (2 \omega t)\right]
\end{aligned}
$$

Finally by using the Lagrange's equation, it is possible to show that an angular misalignment fault can be modeled with a parametric stiffness and a force component. The expression of the stiffness 
matrix is similar to the development for an asymmetric shaft. The final expression of this stiffness matrix $\mathbf{K}^{m_{2}}$ is defined as follows

$$
\mathbf{K}^{m_{2}}=\frac{1}{2}\left(3 k+k^{\prime}\right) r^{2}\left[\begin{array}{cccc}
0 & & & \\
& 0 & & \\
& & 1 & \\
& & & 1
\end{array}\right]+\frac{1}{2} k^{\prime} r^{2}\left[\begin{array}{cccc}
0 & & & \\
& 0 & & \\
& & \cos (2 \omega t) & \sin (2 \omega t) \\
& \sin (2 \omega t) & -\cos (2 \omega t)
\end{array}\right]
$$

The angular misalignment force is given by

$$
\mathbf{f}^{m_{2}}=-\frac{1}{2} \alpha_{m} r^{2}\left[\begin{array}{lll}
0 & 0 & 3 k+k^{\prime}+k^{\prime} \cos (2 \omega t) \quad k^{\prime} \sin (2 \omega t)
\end{array}\right]^{T}
$$

\subsection{Final expression}

Finally the equation of motion of the complete system, that has been previously defined in Equation (1), is rewritten as

$$
\mathbf{M} \ddot{\mathbf{x}}+\mathbf{D} \dot{\mathbf{x}}+\mathbf{K x}=\mathbf{f}
$$

with

$$
\begin{aligned}
\mathbf{M} & =\mathbf{M}_{0}+\mathbf{M}_{c} \cos (2 \omega t)+\mathbf{M}_{s} \sin (2 \omega t) \\
\mathbf{K} & =\mathbf{K}_{0}+\mathbf{K}_{c} \cos (2 \omega t)+\mathbf{K}_{s} \sin (2 \omega t) \\
\mathbf{D} & =\mathbf{C}+\omega \mathbf{G}
\end{aligned}
$$

$\mathbf{M}, \mathbf{K}$ and $\mathbf{D}$ are the global mass, stiffness and damping matrices. They are determinated by assembling the shaft elementary matrices $\mathbf{M}^{e}, \mathbf{C}^{e}, \mathbf{G}^{e}, \mathbf{K}^{e}, \mathbf{K}^{m_{1}}$ and $\mathbf{K}^{m_{2}}$. $\mathbf{f}$ is defined by assembling the different sources of external forces such as unbalance, misalignment reaction force, bow and gravity.

In this study, the damping is given as follows

$$
\mathbf{C}=\alpha_{d} \mathbf{K}_{0}+\beta_{d} \mathbf{M}_{0}
$$

where $\alpha_{d}$ and $\beta_{d}$ are the damping coefficients and $\mathbf{C}$ is the Rayleigh damping matrix. For the interested reader, it may be remained that the damping is overwhelmingly in the bearings in large machines on oil film bearings. In this case, more complex representations of damping need to be taken into account [18].

\section{Non-linear dynamic response of the rotor system with uncer- tainties}

The fault parameters can be partly unknown, consequently, to model them correctly, the choice has been to take these uncertainties in the model of each one developed in the previous section. Then, the response is uncertain and we chose to model it via the Polynomial Chaos Expansion. So, the aim of this section is to mix the Harmonic Balance Method and the Polynomial Chaos Expansion to obtain the non-linear response of a rotor with uncertain parameters. In a first part the Harmonic 
Balance Method which is one of the mathematical approaches to solve equations with nonlinear terms is presented. In a second part, the random character of the fault parameters will be investigated through stochastic model of each fault. Then a methodology will be proposed to take into account the uncertainties in this kind of problem by using a Polynomial Chaos Expansion with the Harmonic Balance Method.

\subsection{Deterministic problem}

Due to the presence of faults, the rotor system is subjected to periodic excitation. So the response $\mathbf{x}(t)$ and the excitation $\mathbf{f}(t)$ can be expressed as finite Fourier series of order $m$ [19]

$$
\mathbf{x}(t)=\sum_{n=0}^{m}\left(\mathbf{A}_{n} \cos (n \omega t)+\mathbf{B}_{n} \sin (n \omega t)\right)
$$

and

$$
\mathbf{f}(t)=\sum_{n=0}^{m}\left(\mathbf{S}_{n} \cos (n \omega t)+\mathbf{T}_{n} \sin (n \omega t)\right)
$$

Consequently the amplitude of the $n^{\text {th }}$ response is defined as $\left|\mathbf{A}_{n}+i \mathbf{B}_{n}\right|$ with $i$ the complex number.

By substituting the previous equations in the rotor system equations, it leads to solve a linear system of size $(2 m+1) n_{\text {dof }}$ where $n_{\text {dof }}$ is the number of degrees of freedom

$$
\mathbf{L X}=\mathbf{f}
$$

with $\mathbf{L}, \mathbf{X}$ and $\mathbf{f}$ are defined as

$$
\begin{aligned}
& \mathbf{L}=\mathbf{I}_{1}+\mathbf{I}_{2}+\mathbf{I}_{3} \\
& \mathbf{X}=\left[\begin{array}{llllll}
\mathbf{A}_{0} & \mathbf{A}_{1} & \mathbf{B}_{1} & \ldots & \mathbf{A}_{m} & \mathbf{B}_{m}
\end{array}\right]^{T} \\
& \mathbf{f}=\left[\begin{array}{lllllll}
\mathbf{S}_{0} & \mathbf{S}_{1} & \mathbf{T}_{1} & \ldots & \mathbf{S}_{m} & \mathbf{T}_{m}
\end{array}\right]^{T}
\end{aligned}
$$

$\mathbf{I}_{1}, \mathbf{I}_{2}$ and $\mathbf{I}_{3}$ are respectively the contributions due to the constant terms, the parametric terms of the stiffness and the parametric terms of the mass.

In our study, some coupling terms appear due to the parametric stiffness (see Section 3.1.2). So an excitation of $n \times$ induces an evolution of the $n-2$ and $n+2$ super harmonic responses. Consequently the truncation order of the non-linear response (54) has to be chosen in order to show this phenomenon. In this paper the $m^{\text {th }}$ order is equal to 4 .

In the following sections, definitions of the terms $\mathbf{I}_{1}, \mathbf{I}_{2}$ and $\mathbf{I}_{3}$ are investigated.

\subsubsection{Constant terms - Definition of $I_{1}$}

Considering the constant terms of the left side of the equation of motion of the complete rotor system defined in Equation (49), it leads to the following ormulation

$$
\mathbf{M}_{0} \ddot{\mathbf{x}}+\mathbf{D} \dot{\mathbf{x}}+\mathbf{K}_{0} \mathbf{x}
$$


By using Equations (54-55) in Equation (60) and after calculations, the formulation can be written as the linear expression $\mathbf{I}_{1} \mathbf{X}$ with

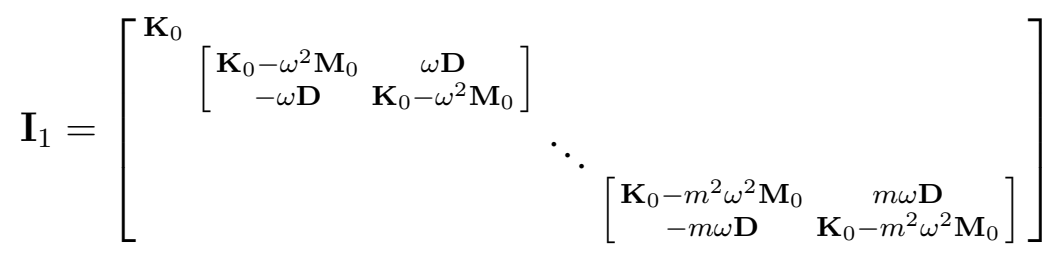

\subsubsection{Parametric terms - Definition of $I_{2}$ and $I_{3}$}

The non-constant terms of the left side of the equation of motion defined in Equation (49) can be rewritten such as follows

$$
\begin{aligned}
& {\left[\mathbf{K}_{c} \cos (2 \omega t)+\mathbf{K}_{s} \sin (2 \omega t)\right] \mathbf{x}} \\
& {\left[\mathbf{M}_{c} \cos (2 \omega t)+\mathbf{M}_{s} \sin (2 \omega t)\right] \ddot{\mathbf{x}}}
\end{aligned}
$$

The displacement $\mathbf{x}$ and the acceleration $\ddot{x}$ can be expressed as truncated Fourier series. By using the definition of $\mathbf{x}(t)$ given in Equation (54) in Equation (62), the parametric stiffness system can be rewritten as

$$
\begin{aligned}
& \sum_{n=0}^{m}\left(\left(\mathbf{K}_{c} \mathbf{A}_{n}+\mathbf{K}_{s} \mathbf{B}_{n}\right) \cos ((n-2) \omega t)+\left(\mathbf{K}_{c} \mathbf{A}_{n}-\mathbf{K}_{s} \mathbf{B}_{n}\right) \cos ((n+2) \omega t)\right. \\
& \left.\quad+\left(\mathbf{K}_{c} \mathbf{B}_{n}-\mathbf{K}_{s} \mathbf{A}_{n}\right) \sin ((n-2) \omega t)+\left(\mathbf{K}_{c} \mathbf{B}_{n}+\mathbf{K}_{s} \mathbf{A}_{n}\right) \sin ((n+2) \omega t)\right)=\mathbf{0}
\end{aligned}
$$

Considering a truncation of order $m$, the previous system can be written as the linear system $\mathbf{I}_{2} \mathbf{X}=\mathbf{0}$ where

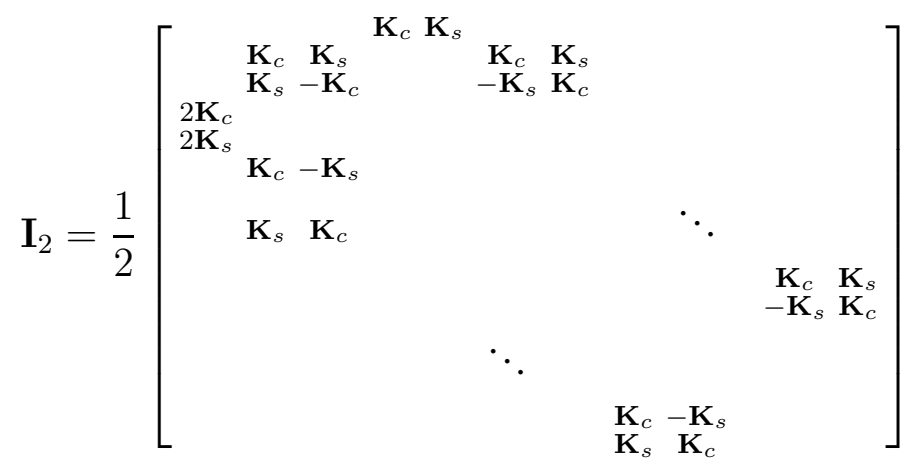

Similarly, using the following expression of the acceleration,

$$
\ddot{\mathbf{x}}(t)=-\omega^{2} \sum_{n=0}^{m} n^{2} \mathbf{A}_{n} \cos (n \omega t)+n^{2} \mathbf{B}_{n} \sin (n \omega t)
$$

one development on the parametric mass system can be done to Equation (63) which leads to linear 
system $\mathbf{I}_{3} \mathbf{X}=\mathbf{0}$ where

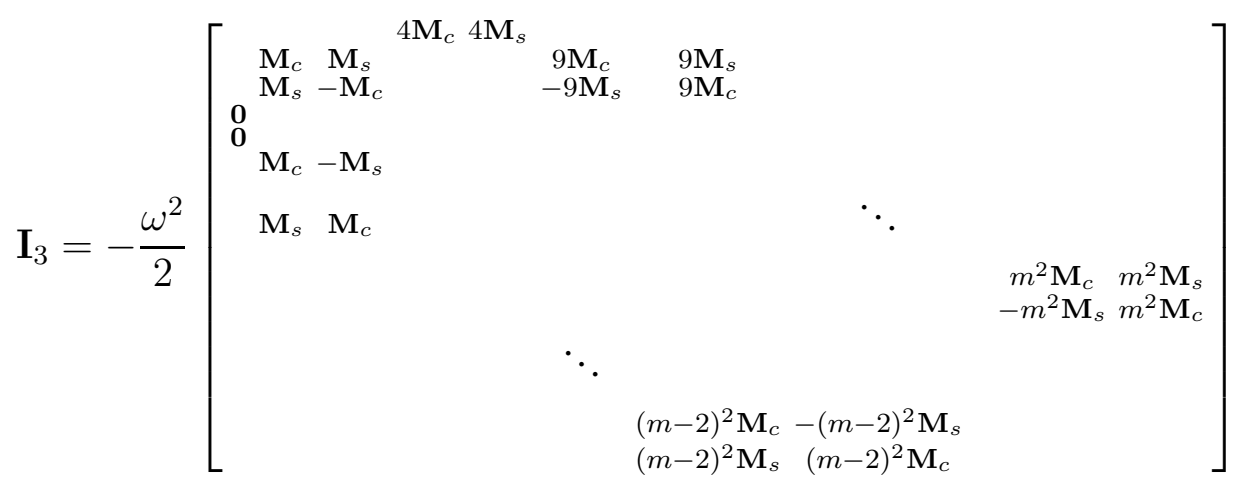

\subsection{Uncertainties on $\mathbf{n} \times$ Harmonic response via the Polynomial Chaos Expan- sion}

Since the material and geometrical properties of the rotor and the excitation force are taken as uncertain, we need to model the response in a stochastic way. Material and geometrical properties are called $\tilde{\mathbf{M}}(\tau), \tilde{\mathbf{D}}(\tau)$ and $\tilde{\mathbf{K}}(\tau)$, the excitation force is written as $\tilde{\mathbf{f}}(\tau)$, then Equation (56) can then be rewritten

$$
\tilde{\mathbf{L}}(\tau) \tilde{\mathbf{X}}(\tau)=\tilde{\mathbf{f}}(\tau)
$$

where argument $\tau$ denotes the random character and

$$
\tilde{\mathbf{L}}(\tau)=\tilde{\mathbf{I}}_{1}(\tau)+\tilde{\mathbf{I}}_{2}(\tau)+\tilde{\mathbf{I}}_{3}(\tau)
$$

and $\tilde{\mathbf{I}}_{i}(\tau), i=1$ to 3 , depend on the material and geometrical properties of the rotor.

Each uncertain quantity (material, geometrical or fault parameter of the rotor) is modeled by implementing the Karhunen-Loeve expansion in the Galerkin formulation of the finite element method [17], it gives :

$$
\tilde{\mathbf{a}}=\overline{\mathbf{a}}+\sum_{l=1}^{L} \xi_{l} \mathbf{a}_{l}
$$

for one uncertain quantity $\tilde{\mathbf{a}}$ (scalar or matrix), where $\left\{\xi_{1}, \ldots \xi_{L}\right\}$ is a set of orthonormal random variables, the notation $\overline{\mathbf{a}}$ refers to the mean of the quantity $\tilde{\mathbf{a}}$ and $\mathbf{a}_{l}$ is its $l$ th Karhunen-Loeve expansion term. Integrating these models into $\tilde{\mathbf{I}}_{i}(\tau)$, quantities $\tilde{\mathbf{L}}(\tau)$ and $\tilde{\mathbf{f}}(\tau)$ can then be expressed on a basis of orthogonal random variables, the Polynomial Chaos basis (details will be given in the following subsection) such that

$$
\begin{aligned}
\tilde{\mathbf{L}}(\tau) & =\sum_{i=0}^{\infty} \mathbf{L}_{i} \Psi_{i}(\underline{\xi}(\tau)), \\
\tilde{\mathbf{f}}(\tau) & =\sum_{j=0}^{\infty} \mathbf{f}_{j} \Psi_{j}(\underline{\xi}(\tau))
\end{aligned}
$$


where $\Psi_{j}(\underline{\xi}(\tau))$ are multidimensional Hermite polynomials and where $\mathbf{L}_{i}(\mathrm{i}=0$ to $\infty)$ are obtained by identification with $\mathbf{I}_{1}(\tau), \mathbf{I}_{2}(\tau)$ and $\mathbf{I}_{3}(\tau)$.

Finally, the response has to be also expanded on the Polynomial Chaos basis such as

$$
\tilde{\mathbf{X}}(\tau)=\sum_{j=0}^{\infty} \mathbf{X}_{j} \Psi_{j}(\underline{\xi}(\tau))
$$

where $\mathbf{X}_{j}$ is the unknown deterministic $j^{\text {th }}$ vector associated with $\Psi_{j}(\underline{\xi}(\tau))$ and $\underline{\xi}=\left\{\xi_{r}\right\}[17,20]$. So the system to be solved, when expanded on the polynomial chaos basis, becomes

$$
\sum_{j=0}^{\infty} \tilde{\mathbf{L}}(\tau) \mathbf{X}_{j} \Psi_{j}(\underline{\xi}(\tau))=\tilde{\mathbf{f}}(\tau)
$$

that can be rewritten as

$$
\left(\sum_{i=0}^{\infty} \mathbf{L}_{i} \Psi_{i}(\underline{\xi}(\tau))\right)\left(\sum_{j=0}^{\infty} \mathbf{X}_{j} \Psi_{j}(\underline{\xi}(\tau))\right)=\sum_{j=0}^{\infty} \mathbf{f}_{j} \Psi_{j}(\underline{\xi}(\tau))
$$

Projecting the system of equations on the subspace spanned by $\left\{\Psi_{k}\right\}_{k=0}^{\infty}$ yields

$$
\sum_{i=0}^{\infty} \sum_{j=0}^{\infty} E\left\{\Psi_{i} \Psi_{j} \Psi_{k}\right\} \mathbf{L}_{i} \mathbf{X}_{j}=\mathbf{f}_{k} E\left\{\Psi_{k}^{2}\right\} \quad k=0,1, \ldots, \infty
$$

where $E\{\}$ denotes the operation of mathematical expectation.

It should be noted that, in practice, the expansion can be truncated after the $P^{\text {th }}$ term. $P$ is the total number of polynomial chaoses used in the expansion excluding the $0^{\text {th }}$ order term and can be determined by $P+1=\frac{(p+r) !}{p ! r !}$ in which $p$ is the order of homogeneous chaos used and $r$ is the number of random variables. Linear system Equation (76) to be solved in practice is then given by

$$
\hat{\mathbf{L}} \hat{\mathbf{X}}=\hat{\mathbf{f}}
$$

with components

$$
\hat{\mathbf{L}}_{j k}=\sum_{i=0}^{P} E\left\{\Psi_{i} \Psi_{j} \Psi_{k}\right\} \mathbf{L}_{i} \quad j, k=0, \ldots, P
$$

and where

$$
\hat{\mathbf{X}}=\left[\begin{array}{lll}
\mathbf{X}_{0} & \ldots & \mathbf{X}_{P}
\end{array}\right]^{T}, \quad \hat{\mathbf{f}}=\left[\begin{array}{lll}
\mathbf{f}_{0} E\left\{\Psi_{0}^{2}\right\} & \ldots & \mathbf{f}_{P} E\left\{\Psi_{P}^{2}\right\}
\end{array}\right]^{T}
$$

It should be noted that coefficients $E\left\{\Psi_{i} \Psi_{j} \Psi_{k}\right\}$ and $E\left\{\Psi_{k}^{2}\right\}$ only have to be calculated once.

\subsection{Uncertainties in multi-faults rotor system}

It is difficult to model accurately faults in a deterministic way. We then chose to model them in a stochastic way. Here, we present one model for each treated fault. 


\subsubsection{Unbalance}

We consider the parameters $m_{e}$ and $\psi_{u}$ as stochastic. From Equation 70, they can be developed as following

$$
\begin{aligned}
& \tilde{m}_{e}(\tau)=\overline{m_{e}}\left(1+\delta_{m} \xi_{1}\right) \\
& \tilde{\psi}_{u}(\tau)=\sigma_{\psi_{u}} \xi_{2}
\end{aligned}
$$

where $\xi_{1}$ and $\xi_{2}$ are Gaussian random variables. Unbalance mass $\tilde{m}_{e}$ is defined by its mean $\bar{m}_{e}$ and its variation coefficient $\delta_{m}$; angular position of the force $\tilde{\psi}_{u}$ has mean 0 and standard deviation $\sigma_{\psi_{u}}$. By substituting Equations (80-81) in Equation (3), also valid in the stochastic domain, unbalance force $\tilde{\mathbf{f}}^{d}$ can be rewritten as

$$
\tilde{\mathbf{f}}^{d}=\overline{m_{e}} d_{e} \omega^{2}\left(1+\delta_{m} \xi_{1}\right)\left[\cos \left(\omega t+\sigma_{\psi_{u}} \xi_{2}\right) \quad \sin \left(\omega t+\sigma_{\psi_{u}} \xi_{2}\right) \quad 0 \quad 0\right]^{T}
$$

Using

$$
\begin{aligned}
& \cos \left(\omega t+\sigma_{\psi_{u}} \xi_{2}\right)=\cos (\omega t) \cos \left(\sigma_{\psi_{u}} \xi_{2}\right)-\sin (\omega t) \sin \left(\sigma_{\psi_{u}} \xi_{2}\right) \\
& \sin \left(\omega t+\sigma_{\psi_{u}} \xi_{2}\right)=\sin (\omega t) \cos \left(\sigma_{\psi_{u}} \xi_{2}\right)+\cos (\omega t) \sin \left(\sigma_{\psi_{u}} \xi_{2}\right)
\end{aligned}
$$

and assuming that

$$
\begin{aligned}
& \cos \left(\sigma_{\psi_{u}} \xi_{2}\right)=\sum_{l=0}^{m}(-1)^{l} \frac{\left(\sigma_{\psi_{u}} \xi_{2}\right)^{2 l}}{(2 l) !} \\
& \sin \left(\sigma_{\psi_{u}} \xi_{2}\right)=\sum_{l=0}^{m}(-1)^{l} \frac{\left(\sigma_{\psi_{u}} \xi_{2}\right)^{2 l+1}}{(2 l+1) !}
\end{aligned}
$$

the unbalance force can be rewritten such as follows

$$
\tilde{\mathbf{f}}^{d}=\sum_{k=0}^{1} \sum_{l=0}^{m} \tilde{\mathbf{f}}_{k l}^{d} \xi_{1}^{k} \xi_{2}^{l}
$$

where $\tilde{\mathbf{f}}_{k l}^{d}$ are the unbalance force components in a basis $\left(\xi_{1}^{k}, \xi_{2}^{l}\right)$ and are defined as

$$
\begin{aligned}
& \tilde{\mathbf{f}}_{k l}^{d}=(-1)^{l}\left(\delta_{m} \xi_{1}\right)^{k} \frac{\left(\sigma_{\psi_{u}} \xi_{2}\right)^{2 l}}{(2 l !)}\left(\left[\begin{array}{llll}
\cos (\omega t) & \sin (\omega t) & 0 & 0
\end{array}\right]^{T}\right. \\
& \left.+\frac{\sigma_{\psi_{u}} \xi_{2}}{2 l+1}[-\sin (\omega t) \quad \cos (\omega t) \quad 0 \quad 0]^{T}\right)
\end{aligned}
$$

Finally, the uncertain loading can be expanded on the polynomial chaos basis as

$$
\tilde{\mathbf{f}}^{d}=\sum_{j=0}^{R} \tilde{\tilde{\mathbf{f}}}_{j}^{d} \Psi_{j}\left(\xi_{1}, \xi_{2}\right)
$$

where $R$ is the number of the polynomial chaoses and parameters $\tilde{\tilde{\mathbf{f}}}_{j}^{d}$ are deduced from $\tilde{\mathbf{f}}_{k l}^{d}$ after one identification procedure between $\Psi_{j}\left(\xi_{1}, \xi_{2}\right)$ basis and $\left(\xi_{1}^{k}, \xi_{2}^{l}\right)$ basis (see Table 3 for the case of TwoDimensional Polynomial Chaoses). 


\begin{tabular}{ccc}
\hline $\mathrm{i}$ & $\mathrm{j}$ & $\xi_{1}^{i} \xi_{2}^{j}$ \\
\hline 0 & 0 & $\Psi_{0}$ \\
1 & 0 & $\Psi_{1}$ \\
0 & 1 & $\Psi_{2}$ \\
2 & 0 & $\Psi_{3}+\Psi_{0}$ \\
1 & 1 & $\Psi_{4}$ \\
0 & 2 & $\Psi_{5}+\Psi_{0}$ \\
3 & 0 & $\Psi_{6}+3 \Psi_{1}$ \\
2 & 1 & $\Psi_{7}+\Psi_{2}$ \\
1 & 2 & $\Psi_{8}+\Psi_{1}$ \\
0 & 3 & $\Psi_{9}+3 \Psi_{2}$ \\
\hline
\end{tabular}

Table 3: Expression of $\xi_{1}^{i} \xi_{2}^{j}$ in polynomial chaos basis for order 2 of the expansion

\subsubsection{Rotor asymmetry}

Material parameters and geometrical properties can be often subject to uncertainties. Then, we can model Young modulus and the stochastic depth of the asymmetry as random.

From Equation (70), we can write :

$$
\tilde{E}(\tau)=\bar{E}\left(1+\delta_{E} \xi_{3}\right)
$$

where $\xi_{3}$ is one Gaussian random variable, $\bar{E}$ and $\delta_{E}$ is the mean and the variation coefficient of the Young modulus respectively.

The uncertain geometrical property considered here is one characteristic of the asymmetric shaft cross section : the depth of the asymmetry, as illustrated in Figure 9.

Then, following again Equation (70), we have :

$$
\tilde{h}(\tau)=\bar{h}\left(1+\delta_{h} \xi_{4}\right)
$$

where $\xi_{4}$ is one random Gaussian variable, $\bar{h}$ and $\delta_{h}$ the mean and the variation coefficient of the depth. Let us denote

$$
h^{\prime}(\tau)=\bar{h} \delta_{h} \xi_{4}
$$

since we can write Equation (91) as $\tilde{h}(\tau)=\bar{h}+h^{\prime} . \bar{h}$ and $h^{\prime}$ are presented in Figure 9 for one $\xi_{4}$ given. Consequently, the moments of inertia are stochastic quantities and are defined by

$$
\tilde{I}_{X}(\tau)=I_{X}+\frac{b h^{\prime 3}}{6}+2 b h^{\prime}\left(R-h-\frac{h^{\prime}}{2}\right), \quad \tilde{I}_{Y}(\tau)=I_{Y}+\frac{h^{\prime} b^{3}}{6}
$$

with

$$
b=2 \sqrt{2 h R-h^{2}}
$$




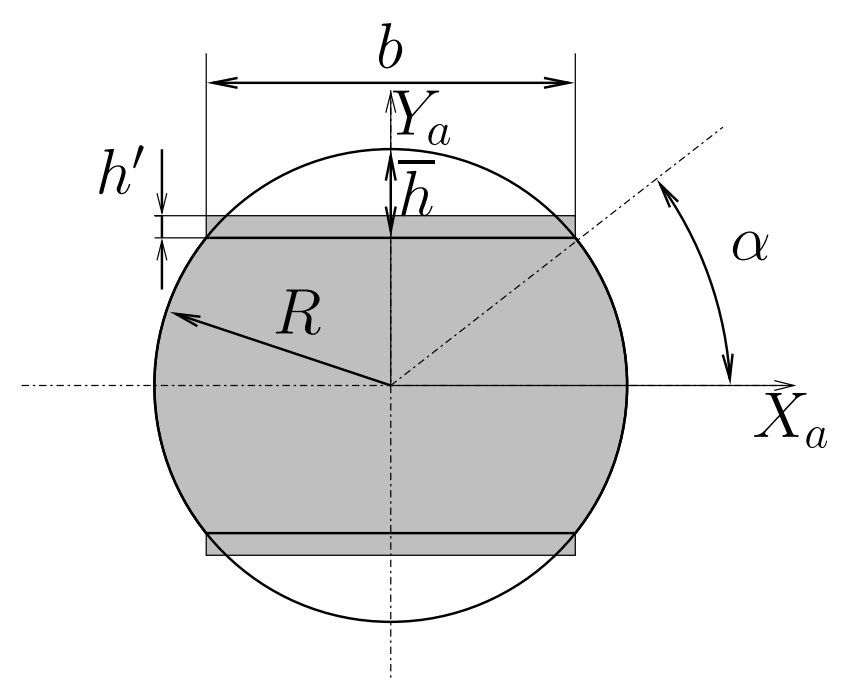

Figure 9: Uncertainty on the shaft section

$I_{X}$ and $I_{Y}$ are deterministic moments defined by Equations (6-7). Substituting Equation (92) in Equation (93) and after developing these equations, we have

$$
\tilde{I}_{X}(\tau)=I_{X}+2 b h(R-h) \delta_{h} \xi_{4}-b h^{2} \delta_{h}{ }^{2} \xi_{4}^{2}+\frac{b h^{3}}{6} \delta_{h}{ }^{3} \xi_{4}^{3}, \quad \tilde{I}_{Y}(\tau)=I_{Y}+\frac{h \delta_{h} b^{3}}{6} \xi_{4}
$$

which can also be expanded as

$$
\tilde{I}_{X}(\tau)=\sum_{i=0}^{3} \tilde{I}_{X_{i}} \xi_{4}^{i}, \quad \tilde{I}_{Y}(\tau)=\sum_{i=0}^{1} \tilde{I}_{Y_{i}} \xi_{4}^{i}
$$

After one identification procedure between Equation (95) and Equation (96), we obtain components $\tilde{I}_{X_{i}}$ and $I_{Y_{i}}$ given by

$$
\begin{aligned}
& \tilde{I}_{X_{0}}=I_{X} \quad, \quad \tilde{I}_{X_{1}}=2 b h(R-h) \delta_{h} \quad, \quad \tilde{I}_{X_{2}}=-b h^{2} \delta_{h}{ }^{2} \quad, \quad \tilde{I}_{X_{3}}=\frac{b h^{3}}{6} \delta_{h}{ }^{3} \\
& \tilde{I}_{Y_{0}}=I_{Y} \quad, \quad \tilde{I}_{Y_{1}}=\frac{h \delta_{h} b^{3}}{6} .
\end{aligned}
$$

It yields the stochastic expressions of the mean and deviatoric moments of inertia

$$
\begin{aligned}
& \tilde{I}_{m}(\tau)=\tilde{I}_{X}(\tau)+\tilde{I}_{Y}(\tau)=\sum_{i=0}^{3}\left(\tilde{I}_{X_{i}}+\tilde{I}_{Y_{i}}\right) \xi_{4}^{i}=\sum_{i=0}^{3} \tilde{I}_{m_{i}} \xi_{4}^{i} \\
& \tilde{I}_{d}(\tau)=\tilde{I}_{X}(\tau)-\tilde{I}_{Y}(\tau)=\sum_{i=0}^{3}\left(\tilde{I}_{X_{i}}-\tilde{I}_{Y_{i}}\right) \xi_{4}^{i}=\sum_{i=0}^{3} \tilde{I}_{d_{i}} \xi_{4}^{i}
\end{aligned}
$$


Introducing stochastic expressions $\tilde{E}, \tilde{I}_{m}$ and $\tilde{I}_{d}$ (Equations (90), (99), (100)) in the deterministic expressions of the elementary stiffness matrices given by Equations (14-16) and also valid for stochastic quantities, the stochastic elementary stiffness matrices are given by

$$
\tilde{\mathbf{K}}_{0}^{e}=\sum_{i=0}^{3} \sum_{j=0}^{1} \tilde{\mathbf{K}}_{0_{i j}}^{e} \xi_{3}^{j} \xi_{4}^{i}, \quad \tilde{\mathbf{K}}_{c}^{e}=\sum_{i=0}^{3} \sum_{j=0}^{1} \tilde{\mathbf{K}}_{c_{i j}}^{e} \xi_{3}^{j} \xi_{4}^{i}, \quad \tilde{\mathbf{K}}_{s}^{e}=\sum_{i=0}^{3} \sum_{j=0}^{1} \tilde{\mathbf{K}}_{s_{i j}}^{e} \xi_{3}^{j} \xi_{4}^{i}
$$

with

$$
\tilde{\mathbf{K}}_{0_{i j}}^{e}=\bar{E} \delta_{E}^{j} \tilde{I}_{m_{i}} \frac{\mathbf{K}_{0}^{e}}{E I_{m}}, \quad \tilde{\mathbf{K}}_{c_{i j}}^{e}=\bar{E} \delta_{E}^{j} \tilde{I}_{d_{i}} \frac{\mathbf{K}_{c}^{e}}{E I_{d}}, \quad \tilde{\mathbf{K}}_{s_{i j}}^{e}=\bar{E} \delta_{E}^{j} \tilde{I}_{d_{i}} \frac{\mathbf{K}_{s}^{e}}{E I_{d}}
$$

Then, after assembling the elementary matrices, the stiffness matrices of the structure are given by

$$
\tilde{\mathbf{K}}_{0}=\sum_{i=0}^{3} \sum_{j=0}^{1} \tilde{\mathbf{K}}_{0_{i j}} \xi_{3}^{j} \xi_{4}^{i}, \quad \tilde{\mathbf{K}}_{c}=\sum_{i=0}^{3} \sum_{j=0}^{1} \tilde{\mathbf{K}}_{c_{i j}} \xi_{3}^{j} \xi_{4}^{i}, \quad \tilde{\mathbf{K}}_{s}=\sum_{i=0}^{3} \sum_{j=0}^{1} \tilde{\mathbf{K}}_{s_{i j}} \xi_{3}^{j} \xi_{4}^{i}
$$

where the components $\tilde{\mathbf{K}}_{0_{i j}}, \tilde{\mathbf{K}}_{c_{i j}}$ and $\tilde{\mathbf{K}}_{s_{i j}}$ are constructed by assembling the matrices $\tilde{\mathbf{K}}_{0_{i j}}^{e}, \tilde{\mathbf{K}}_{c_{i j}}^{e}$ and $\tilde{\mathbf{K}}_{s_{i j}}^{e}$.

Rewriting the developments of the stiffness matrices (Equation (103)) onto a polynomial chaos basis yields

$$
\tilde{\mathbf{K}}_{0}=\sum_{j=0}^{n} \tilde{\tilde{\mathbf{K}}}_{0_{j}} \Psi_{j}\left(\xi_{3}, \xi_{4}\right), \quad \tilde{\mathbf{K}}_{c}=\sum_{j=0}^{n} \tilde{\tilde{\mathbf{K}}}_{c_{j}} \Psi_{j}\left(\xi_{3}, \xi_{4}\right), \quad \tilde{\mathbf{K}}_{s}=\sum_{j=0}^{n} \tilde{\tilde{\mathbf{K}}}_{s_{j}} \Psi_{j}\left(\xi_{3}, \xi_{4}\right)
$$

where $n$ is the number of Hermite polynomials. $\tilde{\tilde{\mathbf{K}}}_{0_{j}}, \tilde{\tilde{\mathbf{K}}}_{c_{j}}$ and $\tilde{\tilde{\mathbf{K}}}_{s_{j}}$ are the rearrangement of $\tilde{\mathbf{K}}_{0_{i j}}, \tilde{\mathbf{K}}_{c_{i j}}$ and $\tilde{\mathbf{K}}_{s_{i j}}$ on the polynomial chaos basis and are obtain after one identification procedure between the two bases.

Similarly, we obtain from the deterministic expressions of the elementary mass matrices given by Equations (23-25) and also valid in the stochastic domain, the following expressions of the stochastic mass matrices

$$
\tilde{\mathbf{M}}_{0}^{e}=\sum_{i=0}^{3} \tilde{\mathbf{M}}_{0_{i}}^{e} \xi_{4}^{i} \quad, \quad \tilde{\mathbf{M}}_{c}^{e}=\sum_{i=0}^{3} \tilde{\mathbf{M}}_{c_{i}}^{e} \xi_{4}^{i} \quad, \quad \tilde{\mathbf{M}}_{s}^{e}=\sum_{i=0}^{3} \tilde{\mathbf{M}}_{s_{i}}^{e} \xi_{4}^{i}
$$

where coefficients of the expansions are given by

$$
\tilde{\mathbf{M}} \mathbf{r}_{0_{i}}^{e}=\frac{\tilde{I}_{m_{i}}}{I_{m}} \mathbf{M} \mathbf{r}_{0}^{e} \quad, \quad \tilde{\mathbf{M}}_{c_{i}}^{e}=\frac{\tilde{I}_{d_{i}}}{I_{d}} \mathbf{M}_{c}^{e} \quad, \quad \tilde{\mathbf{M}}_{s_{i}}^{e}=\frac{\tilde{I}_{d_{i}}}{I_{d}} \mathbf{M}_{s}^{e}
$$

After assembly and identification procedures, the mass matrices can completely be defined by

$$
\tilde{\mathbf{M}}_{0}=\sum_{j=0}^{n} \tilde{\tilde{\mathbf{M}}}_{0_{j}} \Psi_{j}\left(\xi_{3}, \xi_{4}\right) \quad, \quad \tilde{\mathbf{M}}_{c}=\sum_{j=0}^{n} \tilde{\tilde{\mathbf{M}}}_{c_{j}} \Psi_{j}\left(\xi_{3}, \xi_{4}\right) \quad, \quad \tilde{\mathbf{M}}_{s}=\sum_{j=0}^{n} \tilde{\tilde{\mathbf{M}}}_{s_{j}} \Psi_{j}\left(\xi_{3}, \xi_{4}\right)
$$


Finally, the linear system to solve is given by Equation (68) with Equation (69). However, Equation (69) can be rewritten as

$$
\tilde{\mathbf{L}}(\tau)=\sum_{j=0}^{n} \tilde{\tilde{\mathbf{L}}}_{j} \Psi_{j}(\tau)
$$

where $\tilde{\tilde{\mathbf{L}}}_{j}=\tilde{\tilde{\mathbf{I}}}_{1_{j}}+\tilde{\tilde{\mathbf{I}}}_{2_{j}}+\tilde{\tilde{\mathbf{I}}}_{3_{j}}$. Components $\tilde{\tilde{\mathbf{I}}}_{i_{j}}, i=1$ to 3 , can be constructed by one identification procedure between Equation (69) with Equations (61), (65), (67) also valid in the stochastic domain and the following expansions of $\tilde{\mathbf{I}}_{i}, i=1$ to 3 , on the Polynomial Chaos basis :

$$
\tilde{\mathbf{I}}_{1}(\tau)=\sum_{j=0}^{n} \tilde{\tilde{\mathbf{I}}}_{1_{j}} \Psi_{j}\left(\xi_{3}, \xi_{4}\right) \quad, \quad \tilde{\mathbf{I}}_{2}(\tau)=\sum_{j=0}^{n} \tilde{\tilde{\mathbf{I}}}_{2_{j}} \Psi_{j}\left(\xi_{3}, \xi_{4}\right) \quad, \quad \tilde{\mathbf{I}}_{3}(\tau)=\sum_{j=0}^{n} \tilde{\tilde{\mathbf{I}}}_{3_{j}} \Psi_{j}\left(\xi_{3}, \xi_{4}\right)
$$

\subsubsection{Rotor bow}

In this model of bowed shaft, the parameters which seem the most subjected to uncertainties are the amplitude and the phase of the bow. Considering the uncertainties on the phase of the bow, a mathematical development similar to that exposed for the unbalance force can be used. Then, we chose to investigate the random character of the amplitude parameter $\tilde{R}_{0}$ alone defined by

$$
\tilde{R}_{0}(\tau)=\sigma_{R} \xi_{5}
$$

where $\xi_{5}$ is one random Gaussian variable and $\sigma_{R}$ is the standard deviation of the amplitude of the bow. We know that the deterministic equivalent force $\mathbf{f}^{b}$ is expressed as one linear function of $R_{0}$ from Equation (30) with Equations (28) and (29). Therefore, $\tilde{\mathbf{f}}^{b}(\tau)$ verifies the same relation with $\tilde{R}_{0}(\tau)$ in the stochastic domain :

$$
\begin{aligned}
& \mathbf{f}^{b}=\alpha R_{0}, \\
& \tilde{\mathbf{f}}^{b}(\tau)=\alpha \tilde{R}_{0}(\tau)
\end{aligned}
$$

Substituting Equation (110) into Equation (112) and using $\alpha$ from Equation (111) gives

$$
\tilde{\mathbf{f}}^{b}(\tau)=\tilde{\mathbf{f}}_{1}^{b} \xi_{5} \quad \text { with } \quad \tilde{\mathbf{f}}_{1}^{b}=\frac{\sigma_{R}}{R_{0}} \mathbf{f}^{b}
$$

\subsubsection{Misalignment}

Parallel misalignment The uncertain parameter in this modeling is the relative displacement $\tilde{\delta}_{p}$ which can be defined as

$$
\tilde{\delta}_{p}(\tau)=\sigma_{\delta_{p}} \xi_{6}
$$

where $\xi_{6}$ is a random Gaussian variable and $\sigma_{\delta_{p}}$ is the standard deviation of the amplitude of parallel displacement. Substituting Equation (114) in Equation (40), the synchronous force due to the parallel misalignment can be defined by

$$
\tilde{\mathbf{f}}^{m_{1}}(\tau)=\tilde{\mathbf{f}}_{1}^{m_{1}} \xi_{6}
$$


where

$$
\tilde{\mathbf{f}}_{1}^{m_{1}}=N k_{b} \sigma_{\delta_{p}}[\sin (\omega t) \quad 1-\cos (\omega t) \quad 0 \quad 0]^{T}
$$

Angular misalignment Uncertainties can also occur in the determination of the angular fault $\alpha_{m}$ which needs to be modeled in a stochastic way. Let $\xi_{7}$ be one random Gaussian variable, the angular fault $\tilde{\alpha}_{m}$ is defined by

$$
\tilde{\alpha}_{m}(\tau)=\sigma_{\alpha_{m}} \xi_{7}
$$

where $\sigma_{\alpha_{m}}$ is the standard deviation of the angular fault. Finally, substituting Equation (117) in Equation (48), the moment due to the coupling misalignment can be expressed as

$$
\tilde{\mathbf{f}}^{m_{2}}(\tau)=\tilde{\mathbf{f}}_{1}^{m_{2}} \xi_{7}
$$

where

$$
\tilde{\mathbf{f}}_{1}^{m_{2}}=-\frac{1}{2} \sigma_{\alpha_{m}} r^{2}\left[\begin{array}{lll}
0 & 0 & 3 k+k^{\prime}+k^{\prime} \cos (2 \omega t) \quad k^{\prime} \sin (2 \omega t)
\end{array}\right]^{T}
$$

\section{Numerical results}

In this section, the effects of uncertainties on the dynamic response of a rotor system with faults are quantified via the Polynomial Chaos Expansion method. The random dynamic response of the rotor system is defined by its mean value and its variance, besides the envelope of the random response is presented. It is constructed by calculating the maximum and the minimum of all the responses computed by the PCE approach from samples generated following the random law of each input parameter.

The basic deterministic parameters used in all cases are given in Table 1. For the reader comprehension, the initial deterministic rotor system includes multi-faults such as unbalance, asymmetric shaft, bow, parallel and angular misalignments. In addition to this deterministic formulation, uncertainties on the different sources of fault are investigated (see Table 4 for the values of parameters and the order of the PCE taken). For the reference solution computed from the Monte Carlo Simulations the number of samples is taken equal to 1000. The PCE envelope is constructed with 20000 samples. All the results detailed in this section are presented for a rotor frequency evolution from $5 \mathrm{~Hz}$ to 275 Hz with 400 points of discretization.

\subsection{Stiffness uncertainties}

In this part, we consider the uncertainty present on the stiffness properties of the rotor. The variation coefficient on the Young modulus is taken as $\delta_{E}=5 \%$. Computations are done with the MCS and the PCE approaches. Figure 10 presents the mean and variance of the Frequency Response Functions (FRF) of the $n \times$ amplitudes (for $n=1,2,3$ and 4) and the global non-linear response (at node 6 in $x$-direction) for case 1 . It is observed that the mean and variance of the n-FRF, obtained by applying the PCE, are very similar to those of the MCS. Figure 11 presents all the FRF samples obtained by MCS compared to the envelope built by PCE. It can be noted that the contributions of $3 \times$ and $4 \times$ super-harmonic components are very small (respectively $10^{-7} \mathrm{~m}$ and $10^{-9} \mathrm{~m}$ of maximum 


\begin{tabular}{lllllllll}
\hline & $\delta_{E}$ & $\delta_{m}$ & $\sigma_{\psi u}$ & $\delta_{h}$ & $\sigma_{R}$ & $\sigma_{\delta_{p}}$ & $\sigma_{\alpha_{m}}$ & Chaos order \\
\hline Case 1 & $5 \%$ & - & - & - & - & - & - & 2 \\
Case 2 & - & $5 \%$ & $0.06 \mathrm{rad}$ & - & - & - & - & 2 \\
Case 3 & - & - & - & $2 \%$ & - & - & - & 2 \\
Case 4 & - & - & - & - & $10^{-5} \mathrm{~m}$ & - & - & 2 \\
Case 5 & - & - & - & - & - & $10^{-4} \mathrm{~m}$ & - & 2 \\
Case 6 & - & - & - & - & - & - & $5 \times 10^{-4} \mathrm{rad}$ & 2 \\
Case 7 & $2 \%$ & $2 \%$ & $0.03 \mathrm{rad}$ & $1 \%$ & $10^{-5} \mathrm{~m}$ & - & - & 2 \\
Case 8 & $5 \%$ & $5 \%$ & $0.06 \mathrm{rad}$ & $2 \%$ & $10^{-5} \mathrm{~m}$ & $10^{-4} \mathrm{~m}$ & $5 \times 10^{-4} \mathrm{rad}$ & 2 \\
\hline
\end{tabular}

Table 4: Sets of parameters

amplitude), so the global response is mainly due to the $1 \times$ harmonic and the $2 \times$ super-harmonic components (respectively $10^{-2} \mathrm{~m}$ and $10^{-4} \mathrm{~m}$ of maximum amplitude) as illustrated in Figure 11(f). For this example the computational cost of the two methods have been compared and it shows that the Polynomial Chaos Expansion is 40 times faster that the Monte Carlo Simulations.

In this study, the PCE calculations are done with a chaos order equal to 2. It is observed that the difference between both PCE and MCS methods for this order is not significant either for the mean or the variance. However, a better accuracy on the model especially around critical speeds, will be obtained with a higher order than 2 , but it would increase significantly the CPU time. Therefore, we consider the PCE approach with order 2 sufficient and it will be used for all computations.

\subsection{Uncertainties in the unbalance force}

The effects of uncertainties in the unbalance force are now studied. Variation of 5\% on the mass unbalance $\left(\delta_{m}\right)$ and variation of $0.06 \mathrm{rad}$ on the angular position $\left(\psi_{u}\right)$ are considered. Figure 12 shows the mean and the variance of the MCS and the PCE results for the $\mathrm{n} \times$ amplitudes of the rotor system (with $n=1,2,3$ and 4) and the global non-linear response. As previously observed for case 1, the results obtained by the PCE formulation are similar to those of the MCS. Due to the fact that comparisons of the mean and the variance for the MCS and the PCE processes will give the same results for the cases 3, 4, 5 and 6 (i.e. the mean and the variance for the MCS and PCE results will be similar), these representations will not be given in the following part of the paper in order to avoid repetitive informations.

Then, Figure 13 shows the Frequency Response Functions for the global non-linear response and the $\mathrm{n} \times$ super-harmonic component (for $n=1, \ldots, 4$ ) for both MCS and PCE methods. A very good agreement is obtained between the results from the MCS and the PCE: all the MCS results are bounded by the lower and upper envelopes that have been obtained by applying the PCE process. As expected, these random parameters influence mainly the $1 \times$ response. However, due to the coupling terms of asymmetry, evolution of the $3 \times$ super harmonic components is also observed. Indeed, coupling terms between the $1 \times$ and $3 \times$ contributions are presents in the expressions of $I_{2}$ and $I_{3}$ (see Equations (65) and (67)). So a variation on the terms of unbalance causes a variation on the terms of order 3 (see Figure 13(b) and (d)). These facts are also clearly illustrated in Figures 12 

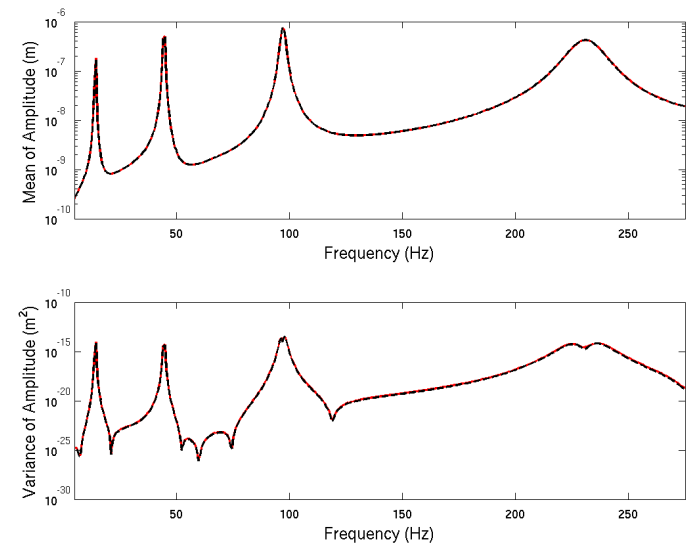

(a)
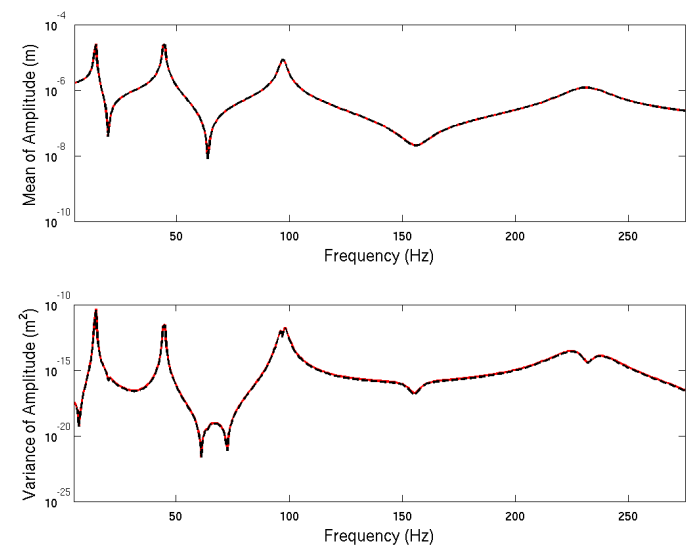

(c)
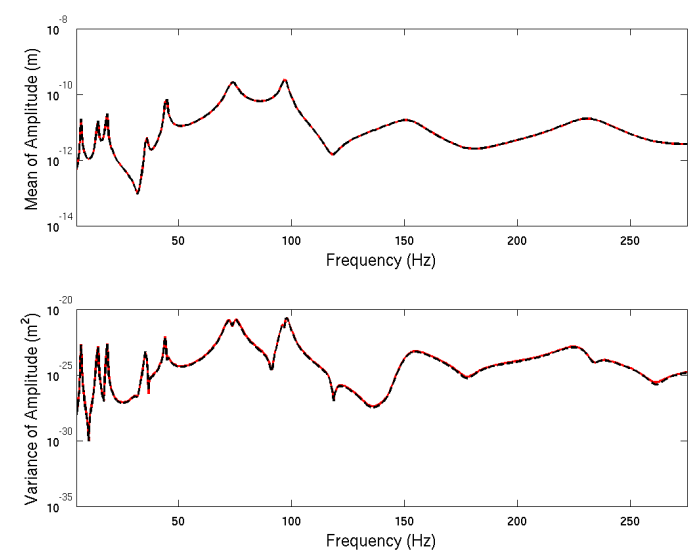

(e)
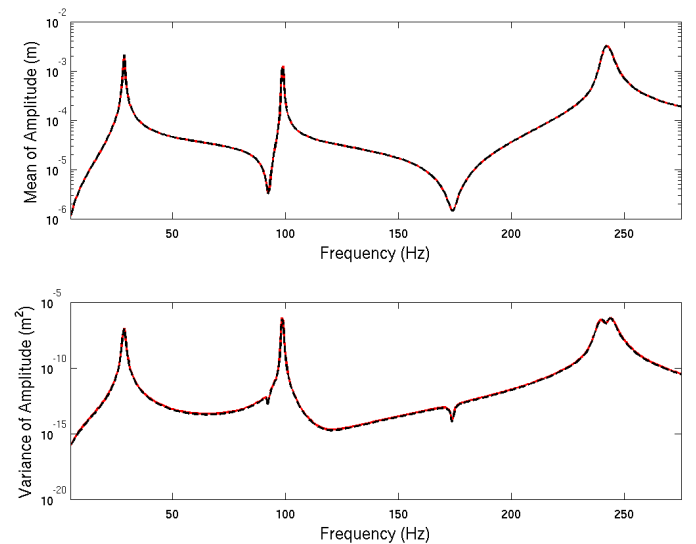

(b)
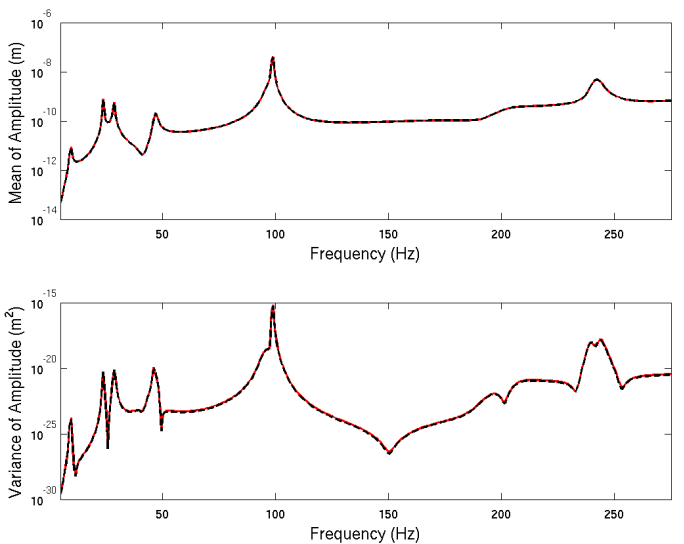

(d)
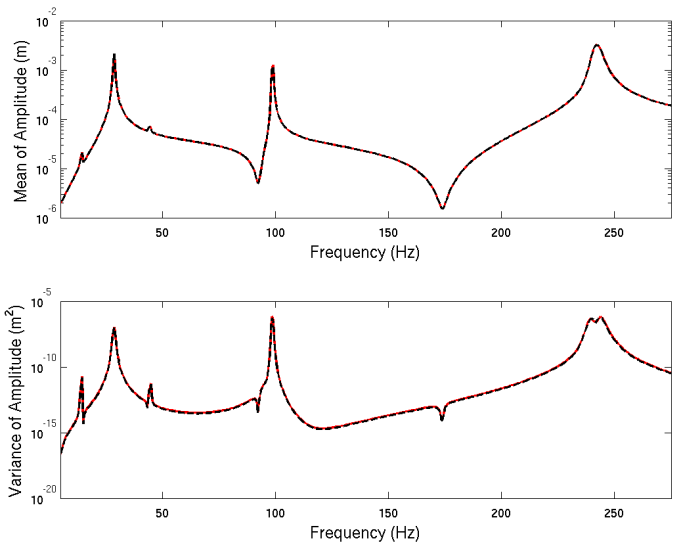

(f)

Figure 10: Frequency Response Functions (Case 1); Mean and Variance of the FRF with PCE (red solid line) and MCS (black dashed line): (a) static component; (b) $1 \times$ harmonic component; (c) $2 \times$ super-harmonic component; (d) $3 \times$ super-harmonic component; (e) $4 \times$ super-harmonic component; (f) global non-linear response. 


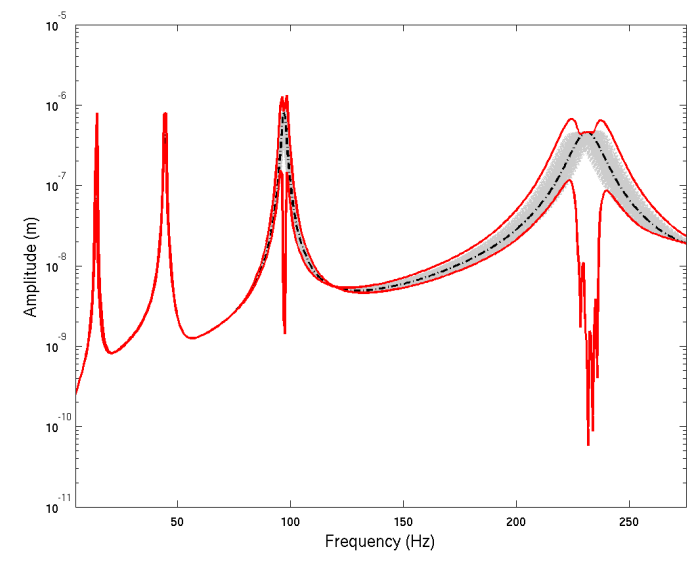

(a)

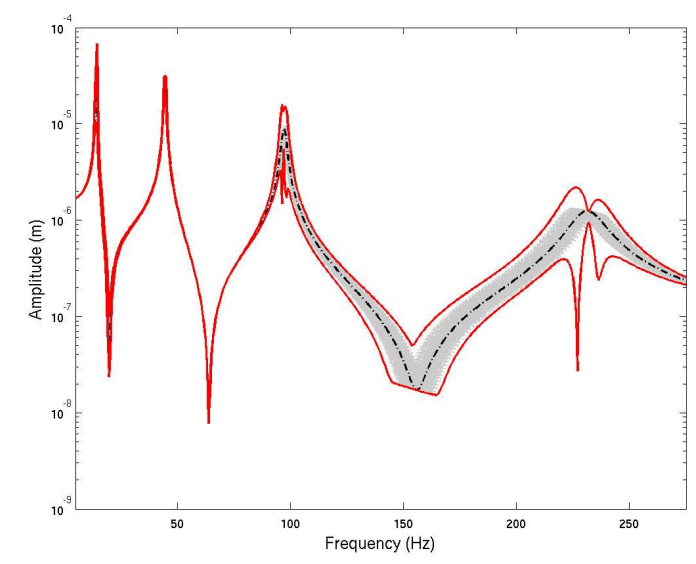

(c)

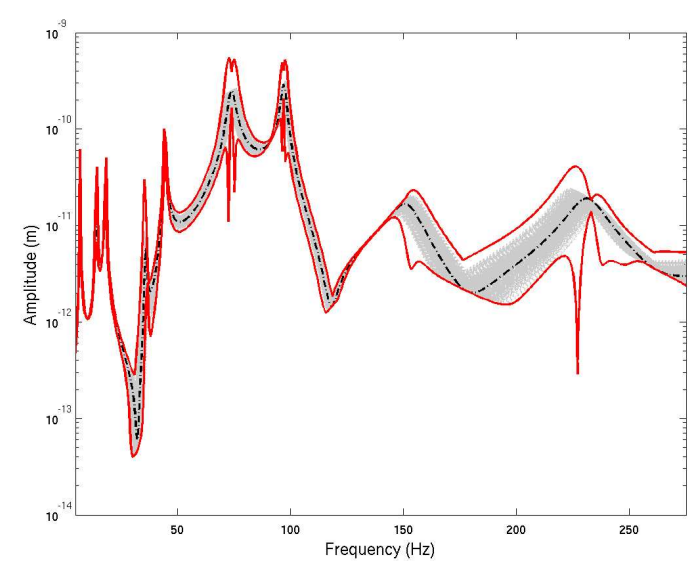

(e)

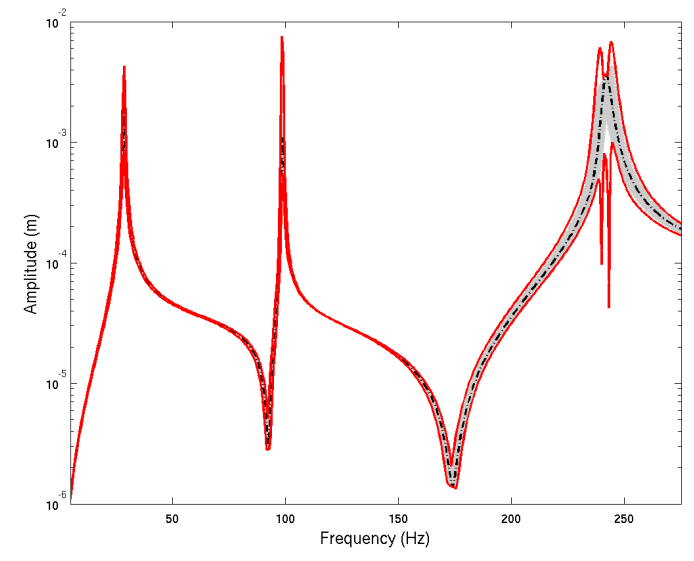

(b)

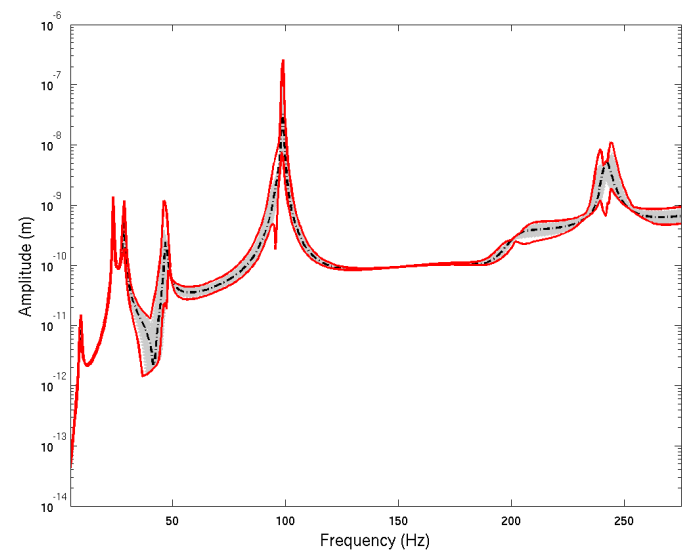

(d)

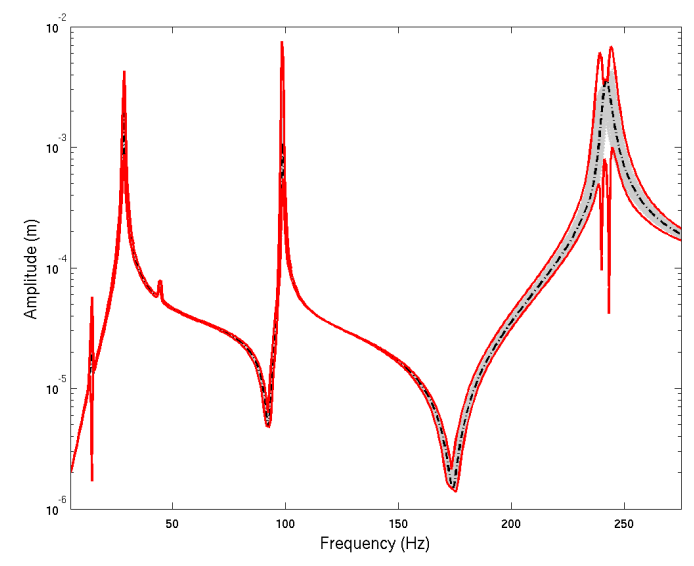

(f)

Figure 11: Frequency Response Functions (Case 1); Lower and upper envelopes (red solid line); MCS (gray dotted line); Deterministic response (black dotted dashed line): (a) static component; (b) $1 \times$ harmonic component; (c) $2 \times$ super-harmonic component; (d) $3 \times$ super-harmonic component; (e) $4 \times$ super-harmonic component; (f) global non-linear response. 
where it is shown that variations of the $\mathrm{n} \times$ amplitudes are only present for the first and third orders of the response. For the static component, the second and fourth orders, the variations of the response amplitudes are negligible (equal to zero if we consider the numerical errors inherent in calculations).

Figure 13(f) indicates also that small variations of the amplitudes around the critical speeds of the $1 \times$ harmonic component are present in the global non-linear response of the rotor system. The range of amplitude is $2.2 \times 10^{-3} \mathrm{~m}$ to $2.7 \times 10^{-3} \mathrm{~m}, 5.4 \times 10^{-4} \mathrm{~m}$ to $1.6 \times 10^{-3} \mathrm{~m}$ and $3.2 \times 10^{-3} \mathrm{~m}$ to $4.0 \times 10^{-3} \mathrm{~m}$ for the first critical speed $(28.7 \mathrm{~Hz})$, the second critical speed $(99.0 \mathrm{~Hz})$ and the third critical speed $(241.8 \mathrm{~Hz})$ respectively.

\subsection{Uncertainties in the asymmetric section properties}

In this subsection, we are interested in the effect of variations on the properties of the shaft crosssection that is through $h$ the depth of the asymmetry and $\delta_{h}$ equal to $3 \%$ is taken. Figure 14 shows the n-FRF for both the Monte Carlo simulations and the lower and upper envelopes that have been calculated by the PCE method. Figures $14(\mathrm{a}), 14(\mathrm{c}), 14(\mathrm{~d})$ and $14(\mathrm{e})$ show variations of the $0 \times, 2 \times, 3 \times$ and $4 \times$ harmonic components. Figure $14($ b) indicates that variations of the $1 \times$ harmonic component are very small. Consequently, Figure 14(e) shows that variations on the global non-linear response are small too due to the fact that $1 \times$ harmonic component represents the predominant vibration of the global non-linear response. Indeed, it is interesting to note here that a variation in the depth of the asymmetry causes significant changes to the contributions of the matrix $I_{2}$ (see Equation (65)), while it has little impact on the responses of order 1 because its contribution in the expression of the matrix $I_{1}$ (see Equation (61)) is not predominant. We recall here that the presence and the variations observed for the $0 \times, 2 \times, 3 \times$ and $4 \times$ harmonics are due to the relations between the different contributions (i.e. static terms and the terms of orders 2, 3 and 4 given in the analytical expressions of Equation (65)).

\subsection{Uncertainties in bow characteristics}

Now, we present the impact of the presence of uncertainties on the parameters characterizing the extended bow. A variation of the bow amplitude $R_{0}$ is considered with a standard deviation $\sigma_{R}$ of $10^{-5} \mathrm{~m}$.

Figure 15 shows the n-FRF for the MCS and the PCE method (with n=1,2,3 and 4). Figures 15(a), 15 (c) and 15 (e) indicate no variability on the $0 \times, 2 \times$ and $4 \times$ super harmonic components whereas Figures 15 (b) and 15 (d) show a high variability on the $1 \times$ and $3 \times$ super harmonic components due to uncertainties in bow characteristics. For the reader comprehension, it is recalled that deterministic multi-faults such as unbalance, asymmetric shaft, bow, parallel and angular misalignments are present in the initial rotor system. Indeed, coupling between the $1 \times$ and $3 \times$ harmonic components exists, as indicated in the expressions of $I_{2}$ and $I_{3}$ (see Equations (65) and (67)). So a variation of the bow characteristics that generates a variation of the $1 \times$ (as previously indicated in Equation (40)) causes also a variation on the terms of order 3 . For example, at the first $1 \times$ critical speed $(28.7 \mathrm{~Hz})$, it is observed variation from $9.3 \times 10^{-5} \mathrm{~m}$ to $4.5 \times 10^{-3} \mathrm{~m}$ for the $1 \times$ harmonic component and variation from $2.4 \times 10^{-11} \mathrm{~m}$ to $1.2 \times 10^{-9} \mathrm{~m}$ for the $3 \times$ harmonic component. Interpretations of these results are similar to the explanations given for unbalance force due to the fact that the forces that model the 

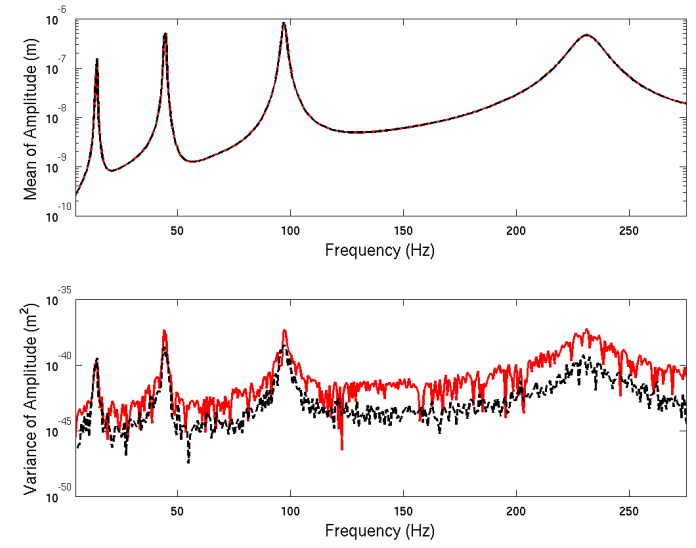

(a)
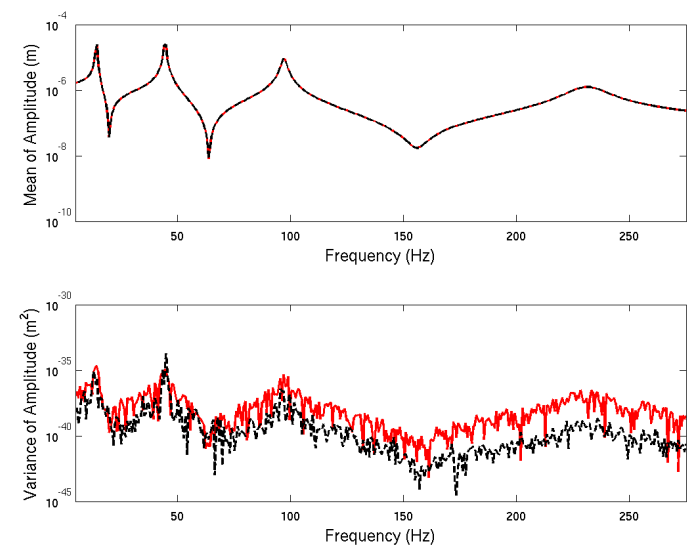

(c)
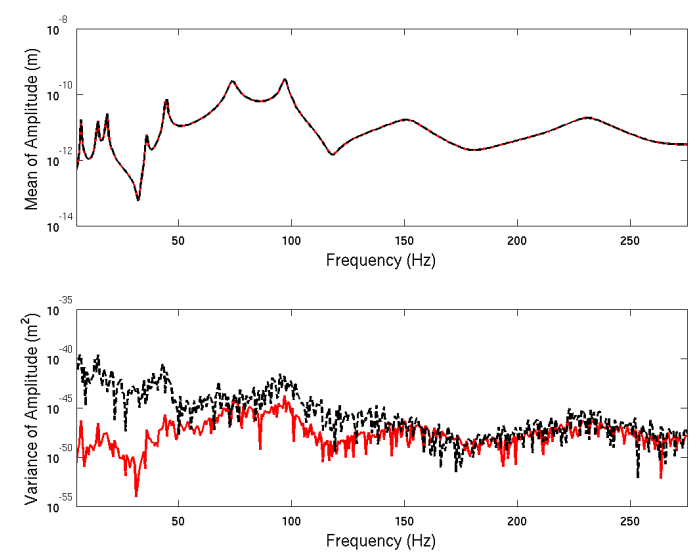

(e)
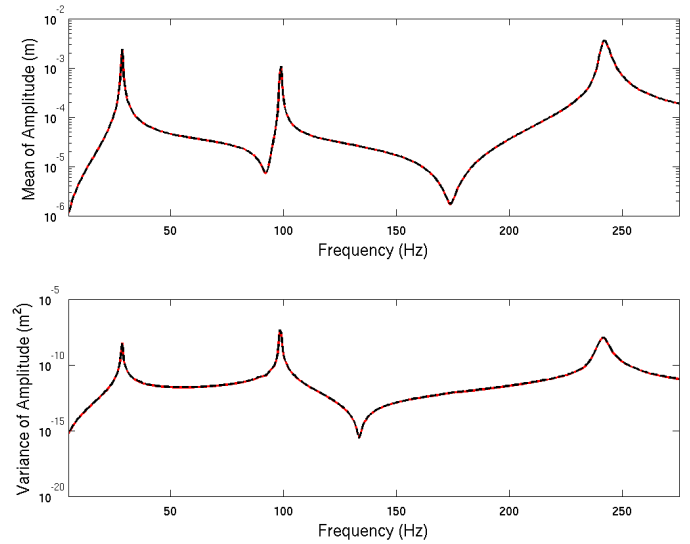

(b)
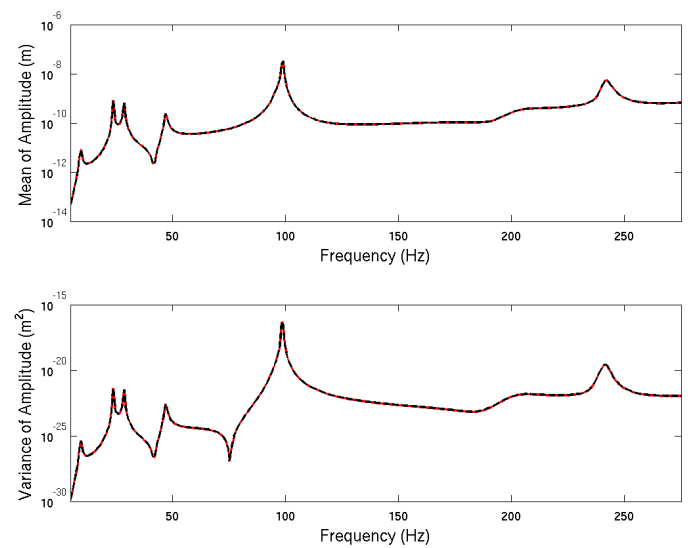

(d)
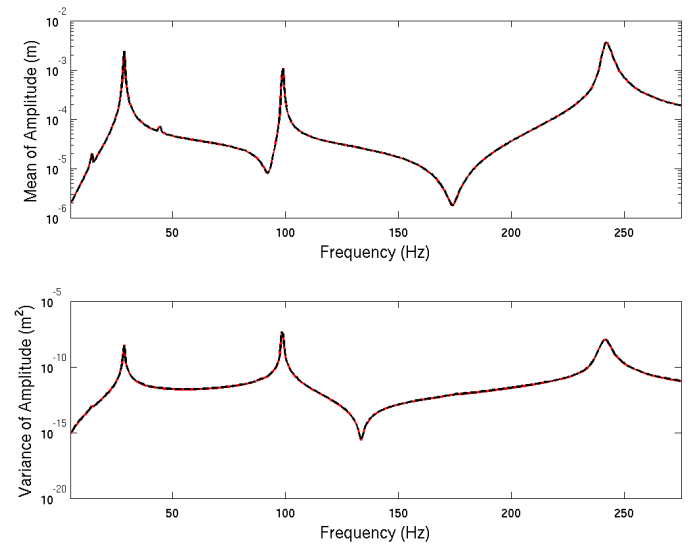

(f)

Figure 12: Frequency Response Functions (Case 2); Mean and Variance of the FRF with PCE (red solid line) and MCS (black dashed line): (a) static component; (b) $1 \times$ harmonic component; (c) $2 \times$ super-harmonic component; (d) $3 \times$ super-harmonic component; (e) $4 \times$ super-harmonic component; (f) global non-linear response. 


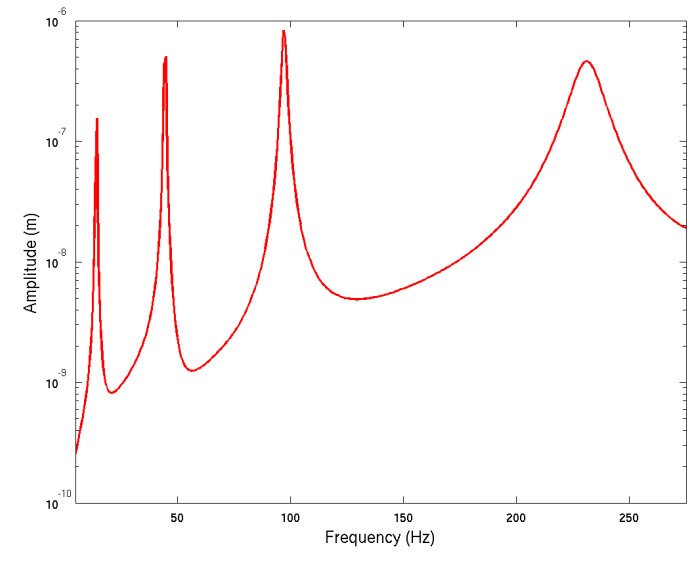

(a)

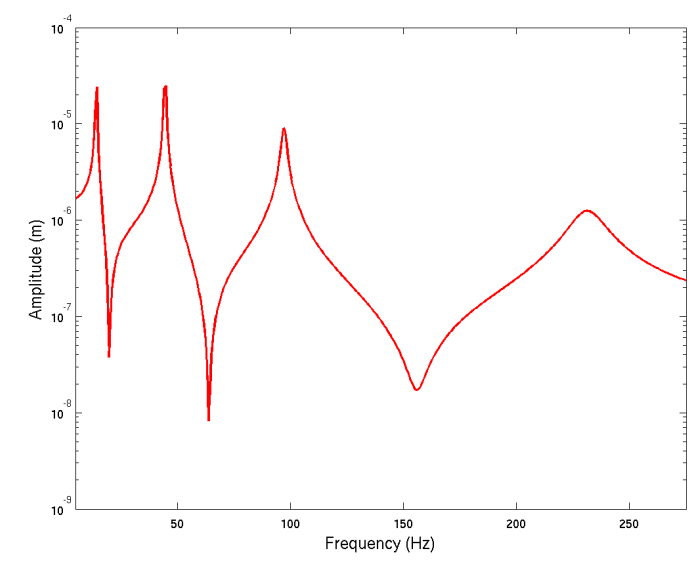

(c)

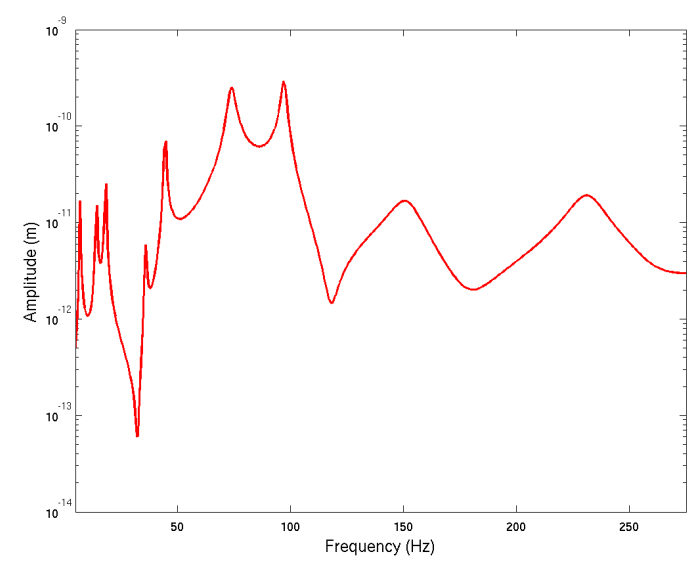

(e)

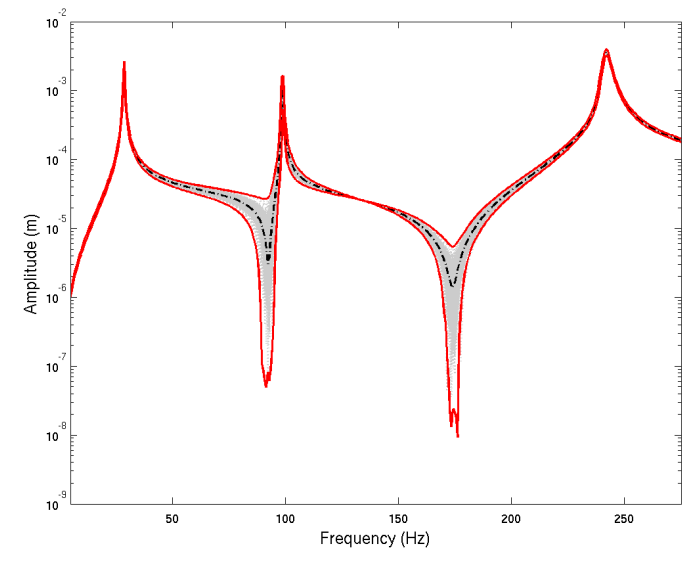

(b)

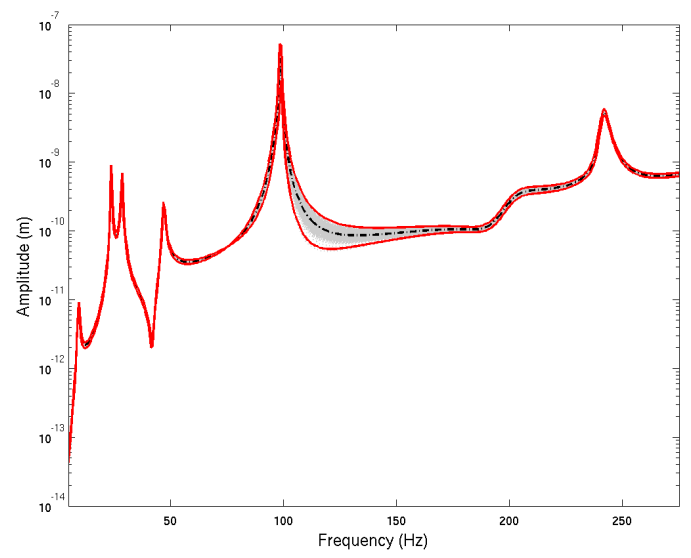

(d)

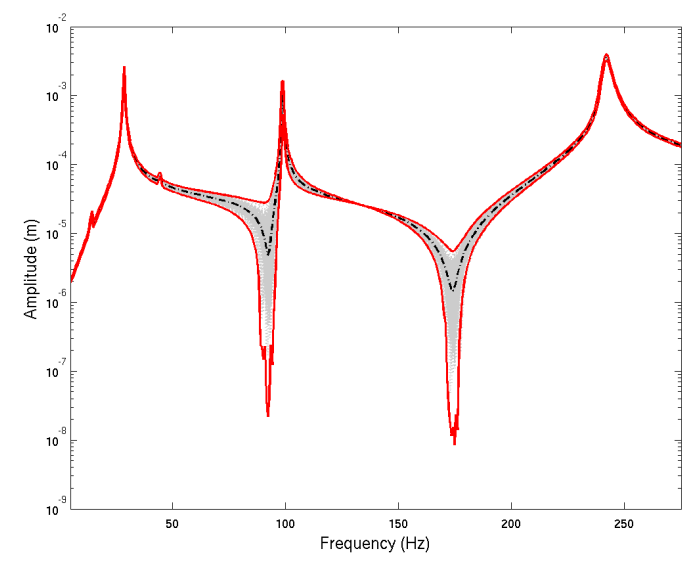

(f)

Figure 13: Frequency Response Functions (Case 2); Lower and upper envelopes (red solid line); MCS (gray dotted line); Deterministic response (black dotted dashed line): (a) static component; (b) $1 \times$ harmonic component; (c) $2 \times$ super-harmonic component; (d) $3 \times$ super-harmonic component; (e) $4 \times$ super-harmonic component; (f) global non-linear response. 


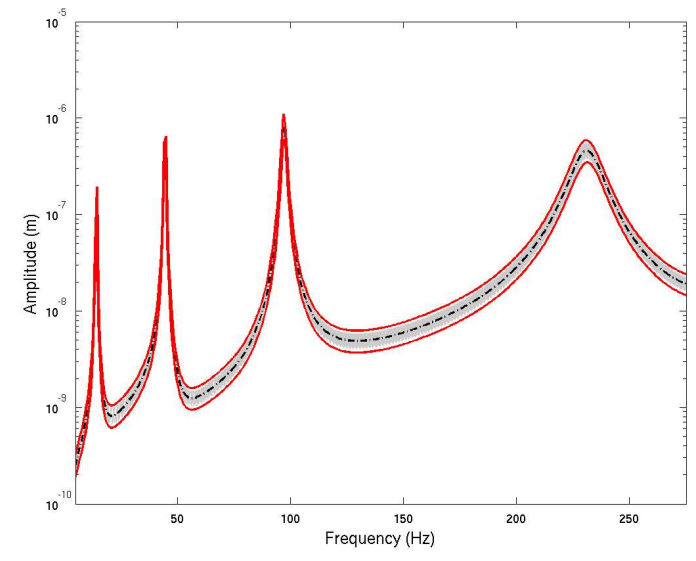

(a)

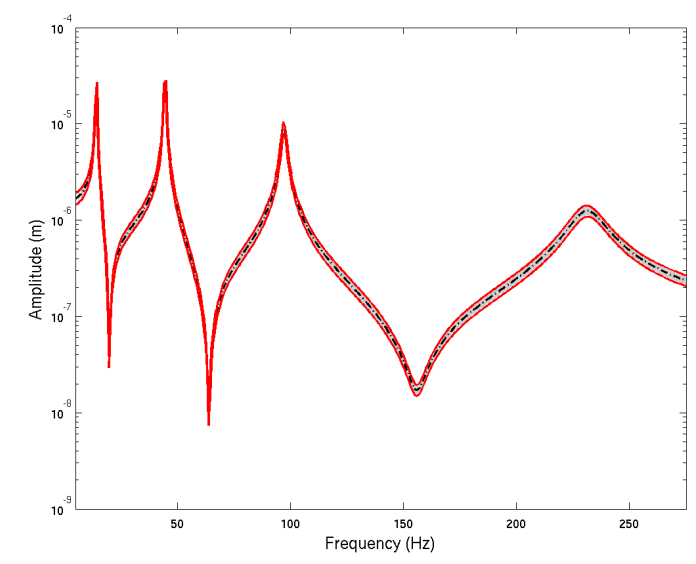

(c)

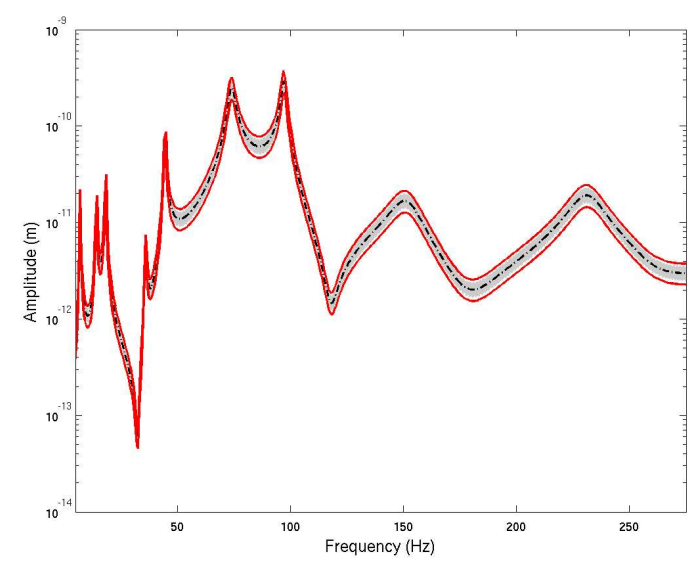

(e)

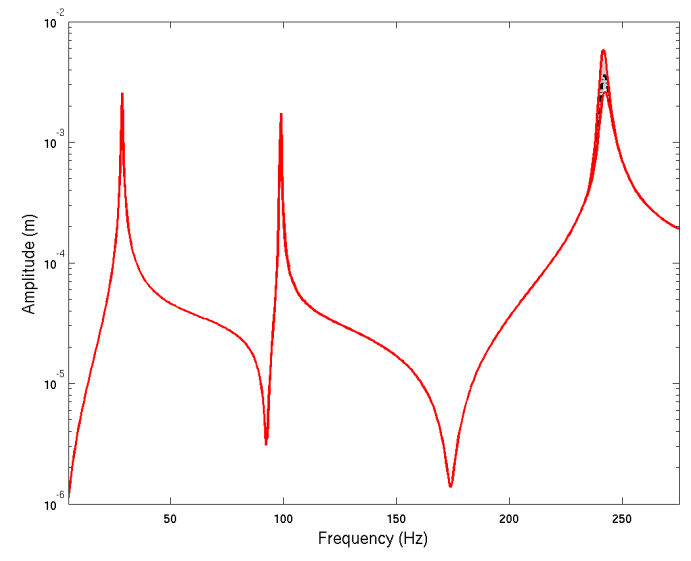

(b)

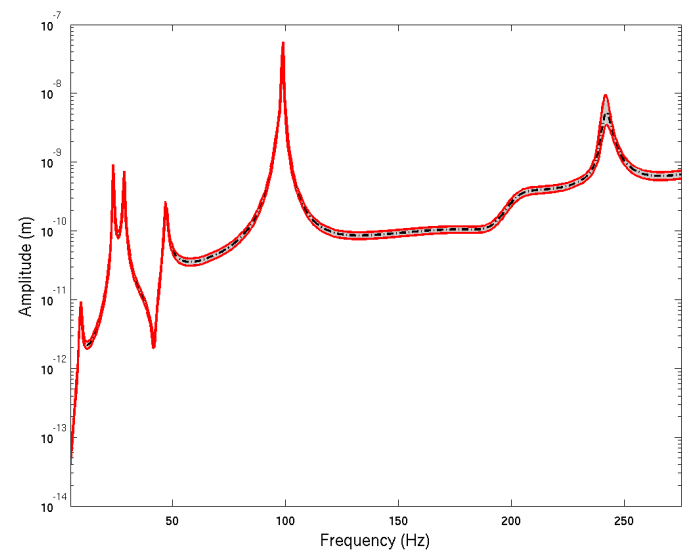

(d)

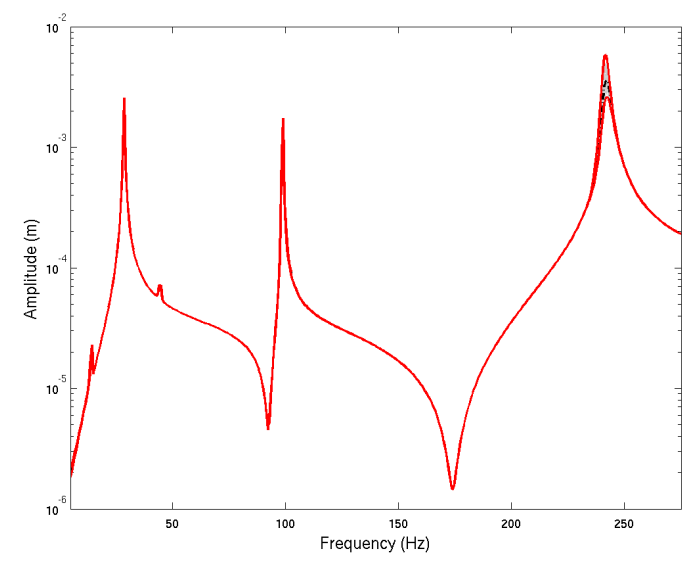

(f)

Figure 14: Frequency Response Functions (Case 3); Lower and upper envelopes (red solid line); MCS (gray dotted line); Deterministic response (black dotted dashed line): (a) static component; (b) $1 \times$ harmonic component; (c) $2 \times$ super-harmonic component; (d) $3 \times$ super-harmonic component; (e) $4 \times$ super-harmonic component; (f) global non-linear response. 
bow are acting as $1 \times$ component and consequently affect the $1 \times$ and $3 \times$ non-linear responses via the coupling terms between odd terms of Equation (65).

Moreover, a high variation of the antiresonance position is observed (as illustrated in Figure 15(b)). It illustrates the fact that the addition of an initial deformation modifies the dynamic response and particularly the position of the minimum of amplitude on the shaft, especially when the initial bow is opposed to the deformation due to unbalance. This phenomenon is illustrated on the first critical speed on the Monte Carlo Simulations : for higher amplitudes than deterministic response, there is no antiresonance (same direction for bow and dynamic deformations) while for lower amplitudes, an antiresonance appears (opposite direction for bow and dynamic deformations).

\subsection{Uncertainties in misalignment}

In this part two cases are studied: the first case deals with an uncertainty on the parallel misalignment fault $\delta_{p}$ for a standard deviation $\sigma_{\delta_{p}}$ of $10^{-4} \mathrm{~m}$ and the second case deals with an angular misalignment $\alpha_{m}$ for a standard deviation $\sigma_{\alpha_{m}}$ of $5 \times 10^{-4} \mathrm{rad}$.

Figure 16 gives the $\mathrm{n} \times$ super harmonic components (with $\mathrm{n}=1,2,3$ and 4 ) and the global non-linear response by using the Monte-Carlo simulations. The upper and lower envelopes that have been calculated by applying the PCE method are also indicated. Firstly, a small variability of the $1 \times$ harmonic component is observed, due to the excitation of the first order given by the parallel misalignment (see Equation 40). Secondly, a very small variation of the Frequency Response Functions of order 3 appears. As explained in the previous paragraphs, the origin of this phenomenon is due to the coupling terms between the first and third orders in the matrices $I_{2}$ and $I_{3}$, as seen in Equations (65) and (67).

Then, Figure 17 shows the $n \times$ harmonic components $(n=1,2,3$ and 4$)$ and the global non-linear response for a parallel misalignment via Monte-Carlo simulations for an angular misalignment. The lower and upper envelopes via the PCE method are also given for all the Frequency Response Functions of the $n \times$ super-harmonic components $(n=1, \ldots, 4)$ and the global non-linear response. Here again, the PCE approach is validated by the Monte-Carlo simulations. It is clearly shown that the $2 \times$ super harmonic component has a high variability due to the fact that the angular misalignment forces are acting as $2 \times$ component (see Equation 48). Moreover, variabilities of the $0 \times$ and $4 \times$ harmonic components can be observed due to the coupling terms in Equations (65) and (67) between the static components, the second and fourth orders.

Finally, differences between the results obtained for the variability of the parallel misalignment and angular misalignment fault are clearly observed (see Figures 16(a), (c) and (e) and Figures 17(a), (c) and (e)). It can be concluded that the variability of an angular misalignment has important effects on the contributions of the $0 \times, 2 \times$, and $4 \times$ super-harmonic components, whereas an parallel misalignment affects only slightly the $1 \times$ responses of the rotor system.

\subsection{Uncertainties in multi-faults}

The main objective of this part of the paper is to demonstrate the efficiency of the new procedure (i.e. the combination of the Harmonic Balance Method and the Stochastic Finite Element Method using the Polynomial Chaos Expansion) when uncertain quantities come from all the parameters simultaneously. Case 7 deals with variations of mechanical properties of the shaft (with a variation 


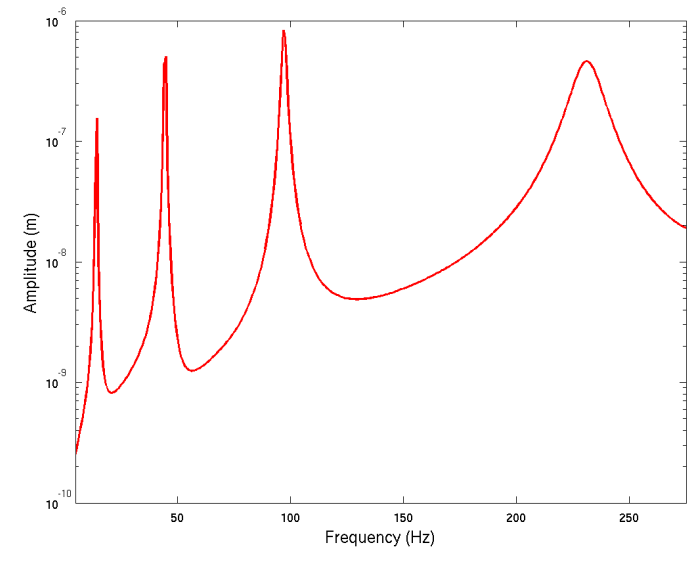

(a)

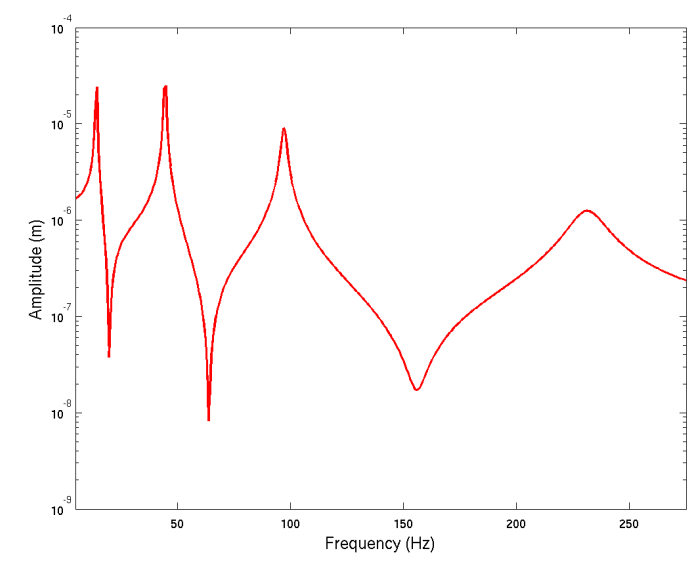

(c)

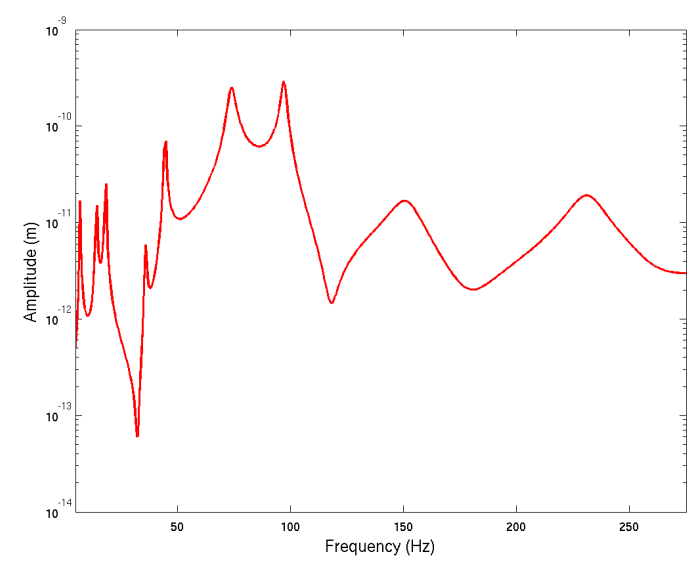

(e)

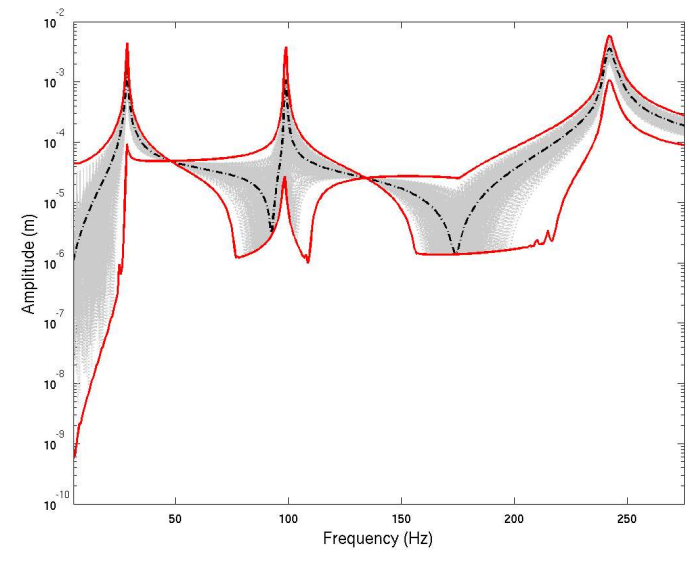

(b)

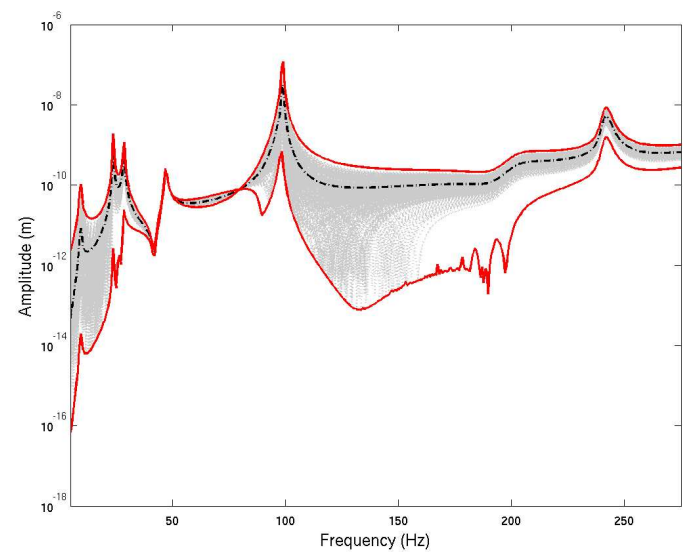

(d)

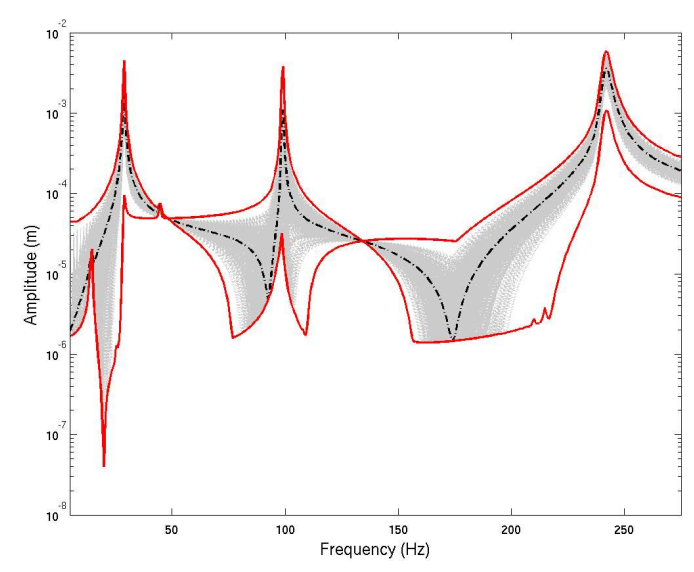

(f)

Figure 15: Frequency Response Functions (Case 4); Lower and upper envelopes (red solid line); MCS (gray dotted line); Deterministic response (black dotted dashed line): (a) static component; (b) $1 \times$ harmonic component; (c) $2 \times$ super-harmonic component; (d) $3 \times$ super-harmonic component; (e) $4 \times$ super-harmonic component; (f) global non-linear response. 


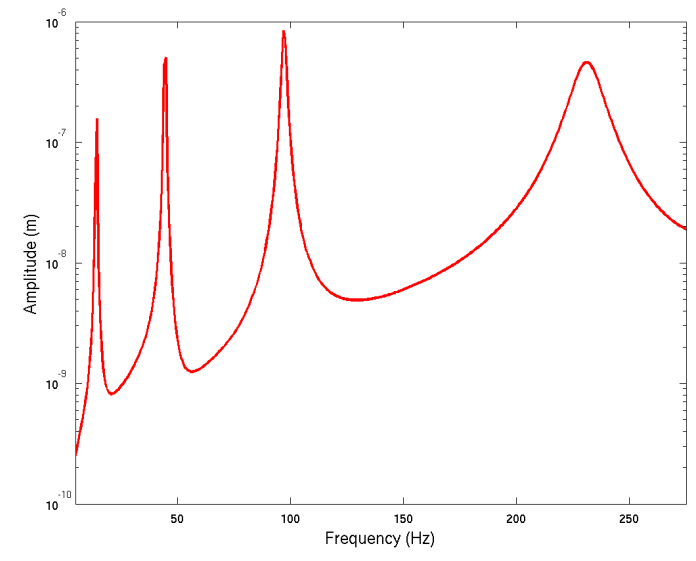

(a)

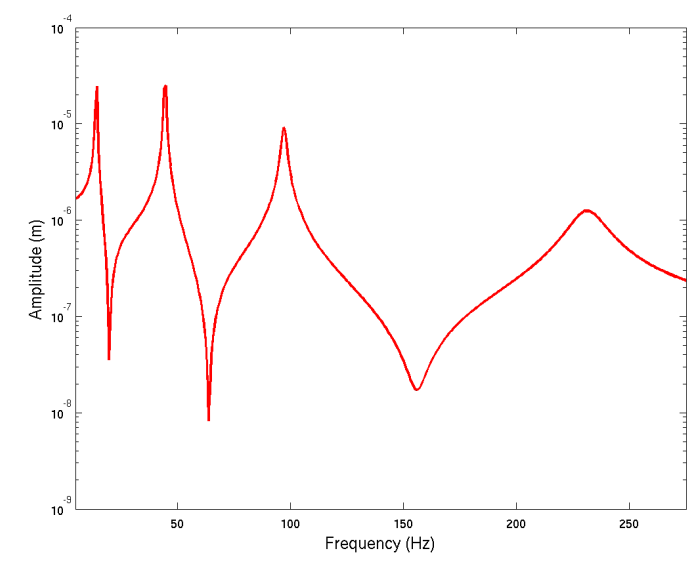

(c)

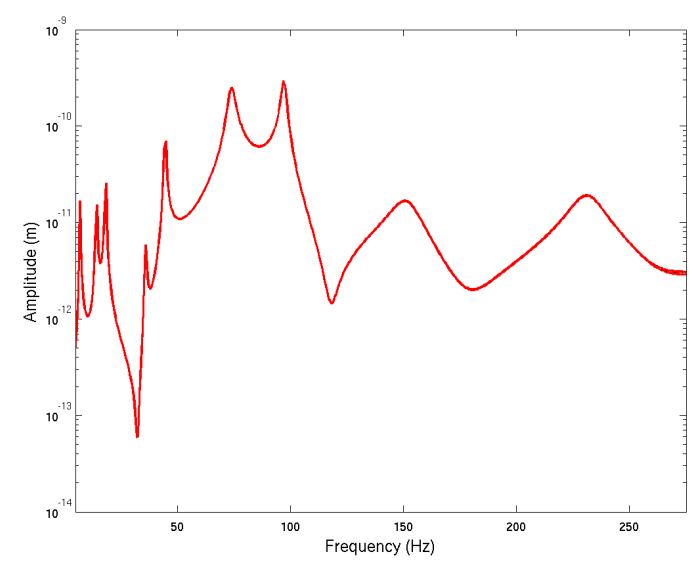

(e)

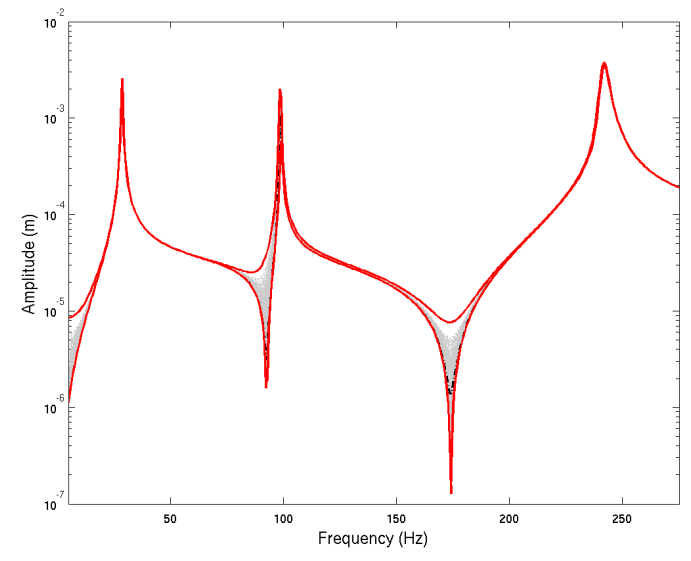

(b)

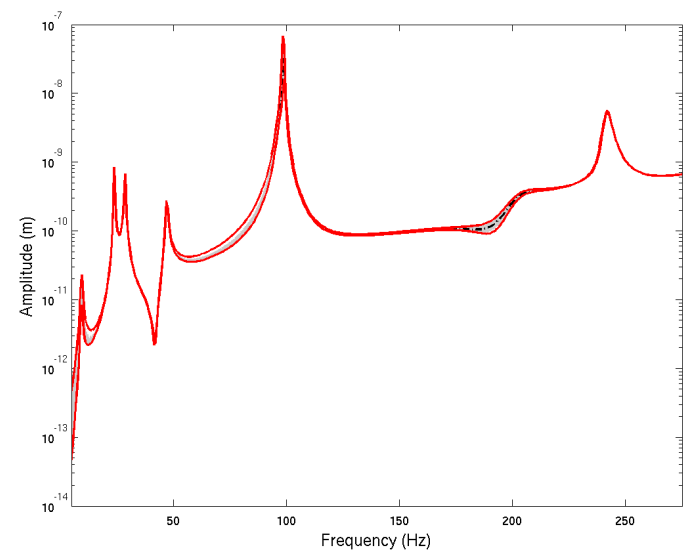

(d)

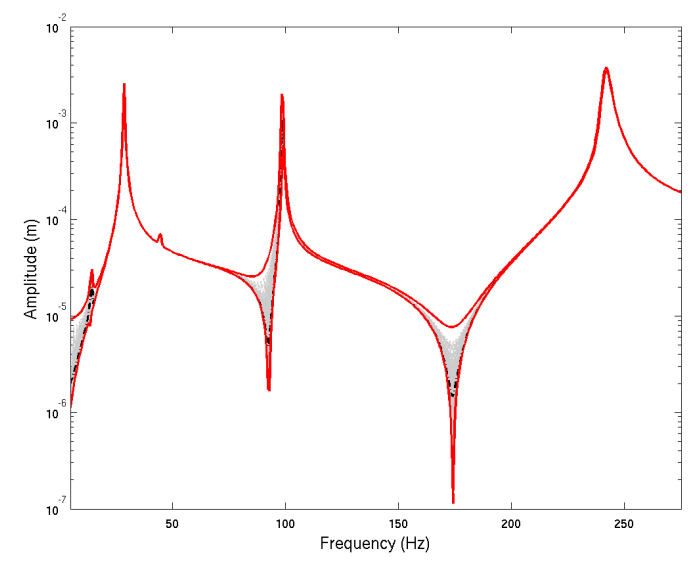

(f)

Figure 16: Frequency Response Functions (Case 5); Lower and upper envelopes (red solid line); MCS (gray dotted line); Deterministic response (black dotted dashed line): (a) static component; (b) $1 \times$ harmonic component; (c) $2 \times$ super-harmonic component; (d) $3 \times$ super-harmonic component; (e) $4 \times$ super-harmonic component; (f) global non-linear response. 


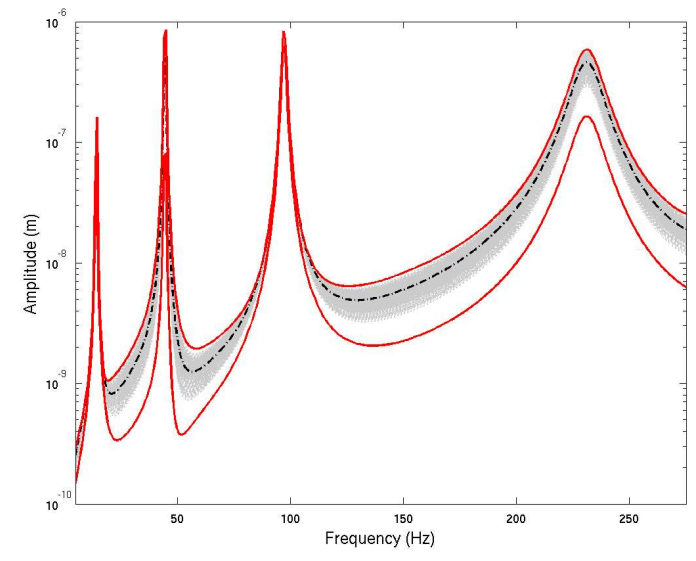

(a)

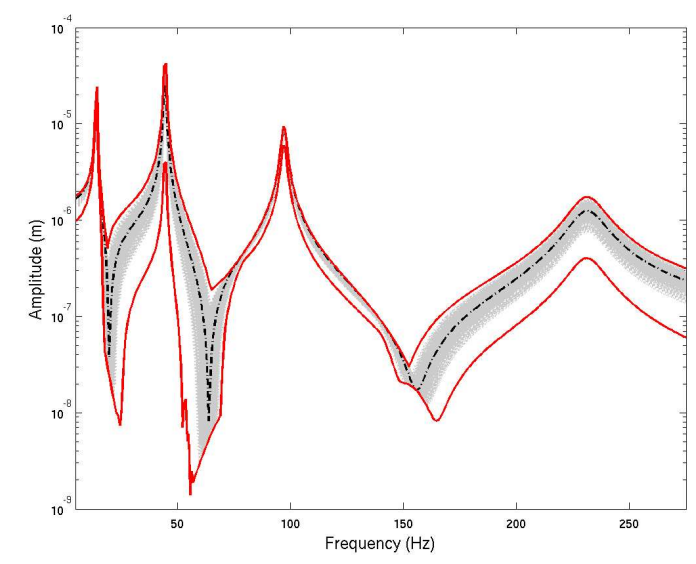

(c)

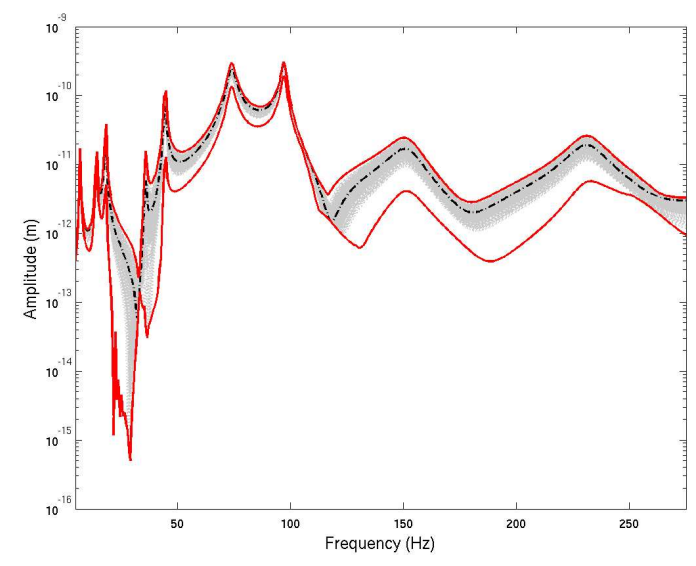

(e)

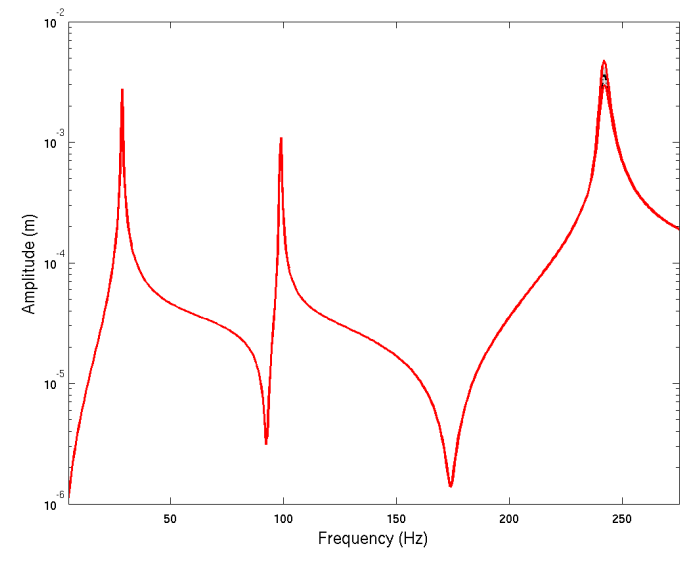

(b)

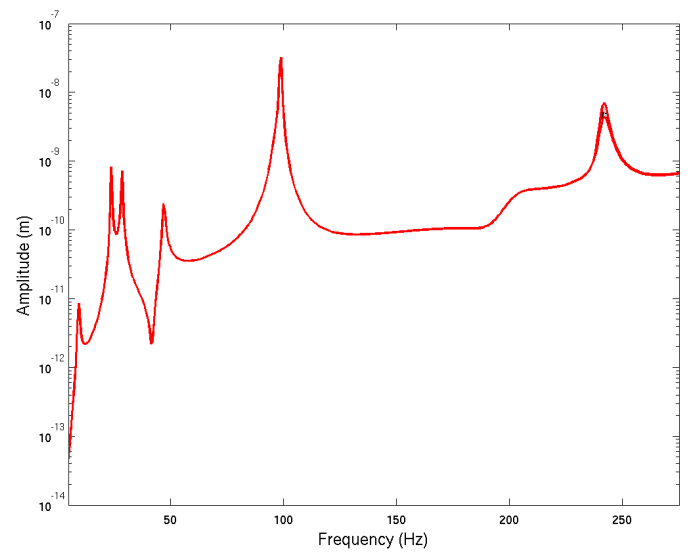

(d)

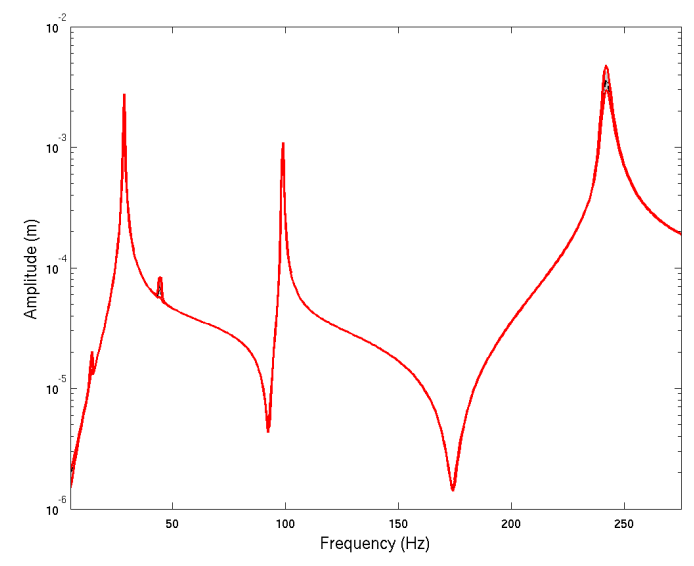

(f)

Figure 17: Frequency Response Functions (Case 6); Lower and upper envelopes (red solid line); MCS (gray dotted line); Deterministic response (black dotted dashed line): (a) static component; (b) $1 \times$ harmonic component; (c) $2 \times$ super-harmonic component; (d) $3 \times$ super-harmonic component; (e) $4 \times$ super-harmonic component; (f) global non-linear response. 
coefficient of the Young modulus $\delta_{E}=2 \%$ ), an unbalance excitation (with a variation coefficient mass unbalance $\delta_{m}=2 \%$ and a standard deviation of the angular position $\sigma_{\psi_{u}}=0.03 \mathrm{rad}$ ) and a bow amplitude (with a standard deviation of the bow $\sigma_{R}=10^{-5} \mathrm{~m}$ ). For the case 8, parallel and angular misalignments of $10^{-4} \mathrm{~m}$ and $5.10^{-4}$ rad respectively are added to the previous case. All the uncertainties on the different sources of fault are given in Table 4 .

The mean and the variance of the $\mathrm{n} \times$ harmonic components ( $\mathrm{n}=1,2,3$ and 4$)$ and the global nonlinear response for both methods MCS and PCE are presented on Figure 18 for case 7 and on Figure 20 for case 8 . Figures 19 and 21 show the n-FRF, the global non-linear response and the upper and lower envelopes via the PCE method, respectively for case 7 and case 8 . The results of the global non-linear amplitudes and the $\mathrm{n} \times$ harmonic components via the Monte-Carlo simulations are also given in gray lines in Figures 19 and 21. These results given for variations on material and geometrical parameters, excitations and faults characteristics show that the mean and the variance of all the responses as well as the upper and lower PCE envelopes agree very well with the Monte Carlo samples. So it proves the efficiency of the PCE method to evaluate the non-linear response of a rotor system in presence of multi-faults such as unbalance, misalignment, bow and asymmetric shaft. For these two examples, the computational cost of the Polynomial Chaos Expansion increases significantly due to the fact there is several random variables. So it increases the size of the Polynomial Chaos basis and consequently the size of the linear system to solve Equation (77). For cases 7 and 8, the chaos order is taken equal to 2 for all random quantities, consequently the Polynomial Chaos basis size is equal to 21 and 36 respectively.

Figures $19(\mathrm{~b}, \mathrm{f})$ and $21(\mathrm{~b}, \mathrm{f})$ show that for these two cases the global non-linear displacement is mainly due to the first harmonic contribution. We recall that the appearances of the various components (i.e. evolutions of the mean and variance for the $1 \times, 2 \times, 3 \times$ and $4 \times$ components) are due to the presence of all the faults in the system, which creates excitations of orders 1 and 2 and the coupling terms in the expressions given in Equations 65 and 67.

By comparing Figures 19 and 21, it appears that differences between these two cases are the more significant for $0 \times, 2 \times$ and $4 \times$ components, whereas the $1 \times$ and $3 \times$ responses seem to be very similar. It is due to the fact that uncertainties on the angular misalignment are added for case 8: as previously seen, an angular misalignment can affect the $0 \times, 2 \times$ and $4 \times$ harmonic components via the expressions of matrices $I_{2}$ and $I_{3}$. For the reader comprehension, it can be noted that, a variability

of $n^{\text {th }}$ order induces a variability of the $(n-2)^{t h}$ th and $(n+2)^{t h}$ orders due to expressions of $I_{2}$ and $I_{3}$ in Equations (65) and (67).

\subsection{Orbits}

In this part of the paper, results are investigated through the orbits of the shaft in order to understand the dynamic behavior of the shaft with the observation of simple, double or complex loops for different rotor frequencies. Orbits are constructed by using displacements in $x$-direction and $y$-direction at the node under study.

Figure 22 shows the evolution of orbits at node 6 for various rotation speeds with a set of random parameters defined in case 7 . It can be noted that the orbits are presented without the static deflection of the rotor in order to show the complexity of the non-linear response that generally occurs at low amplitudes. Figures 22(b), 22(d) and 22(f) show the orbit of the shaft for the first, second and third 

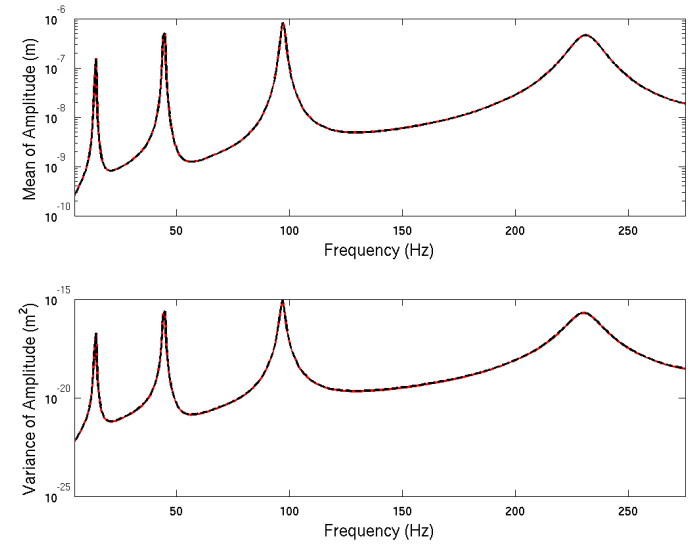

(a)
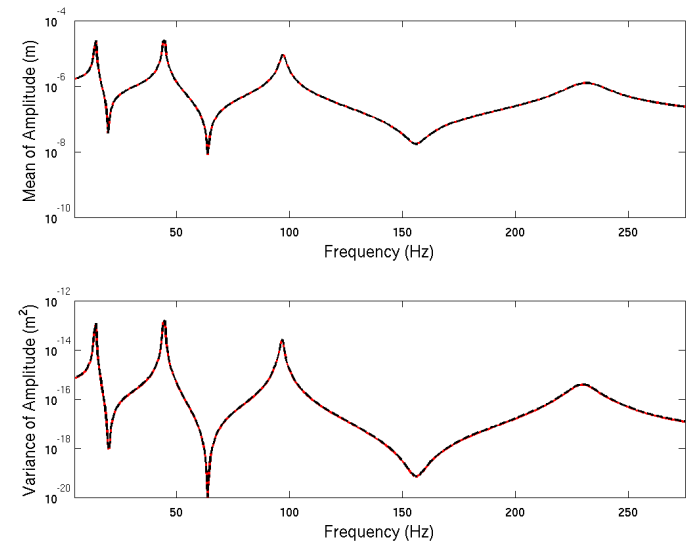

(c)
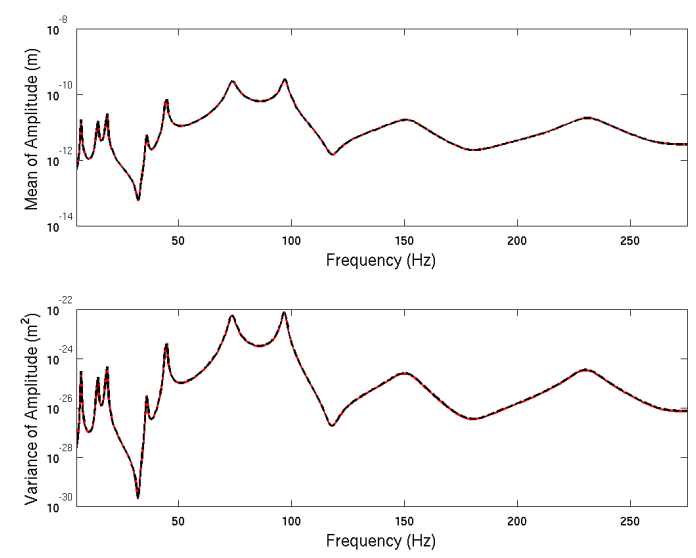

(e)
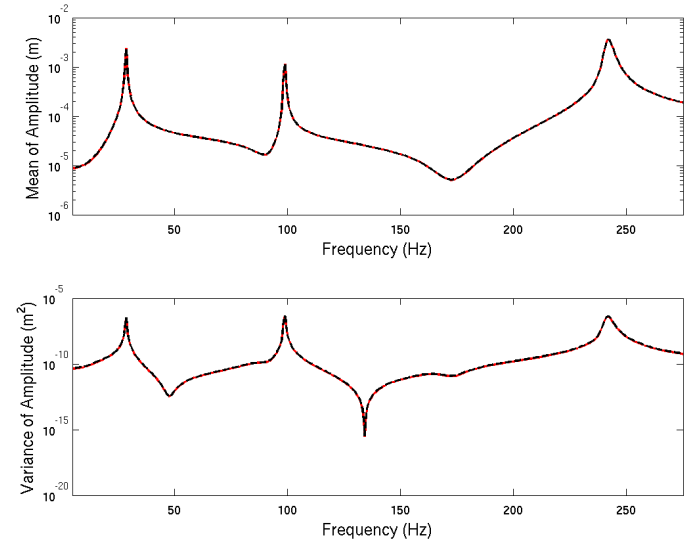

(b)
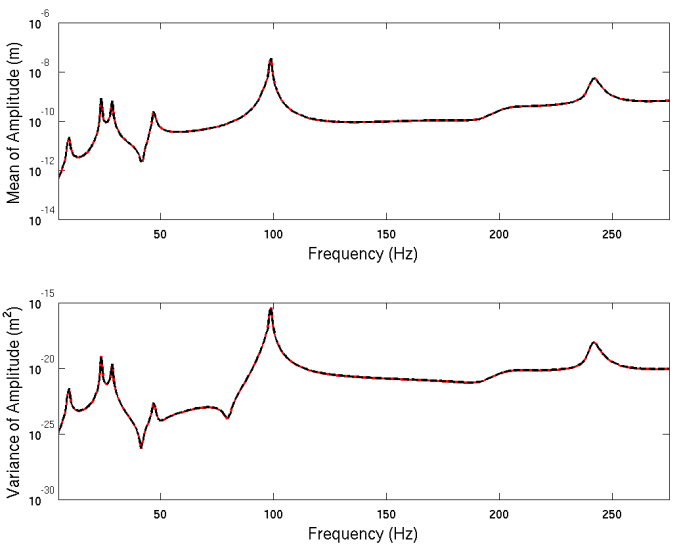

(d)
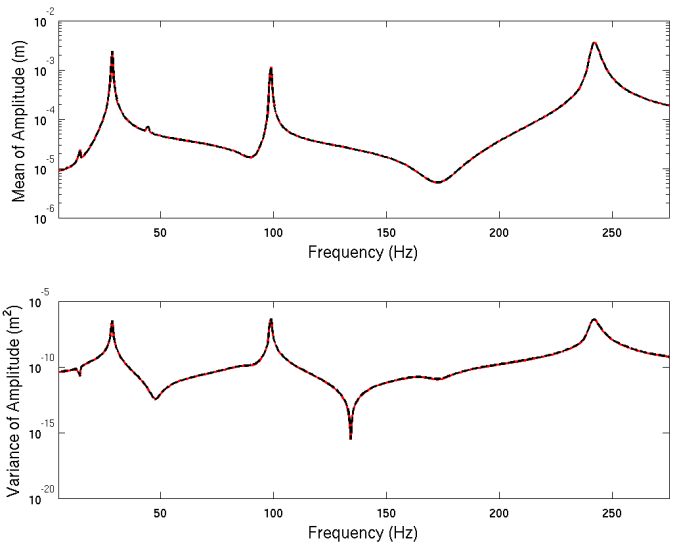

(f)

Figure 18: Frequency Response Functions (Case 7); Mean and Variance of the FRF with PCE (red solid line) and MCS (black dashed line): (a) static component; (b) $1 \times$ harmonic component; (c) $2 \times$ super-harmonic component; (d) $3 \times$ super-harmonic component; (e) $4 \times$ super-harmonic component; (f) global non-linear response. 


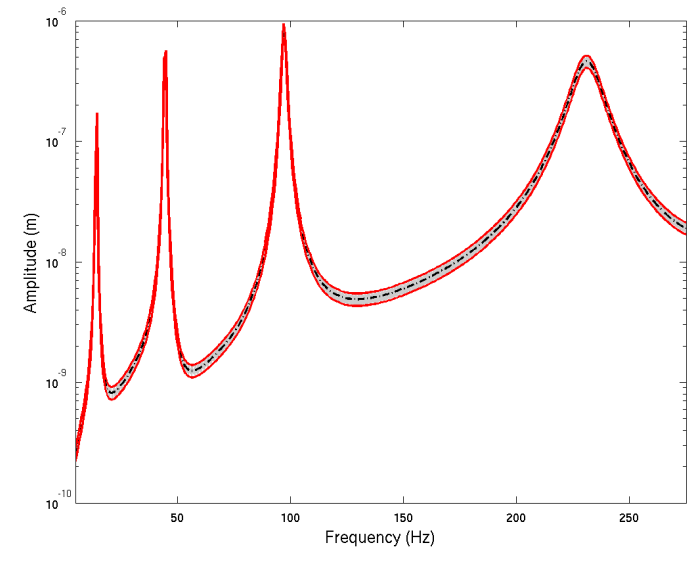

(a)

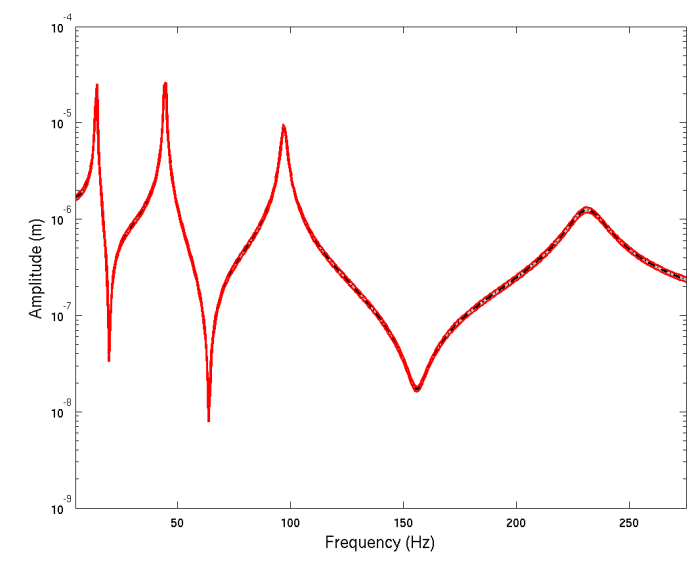

(c)

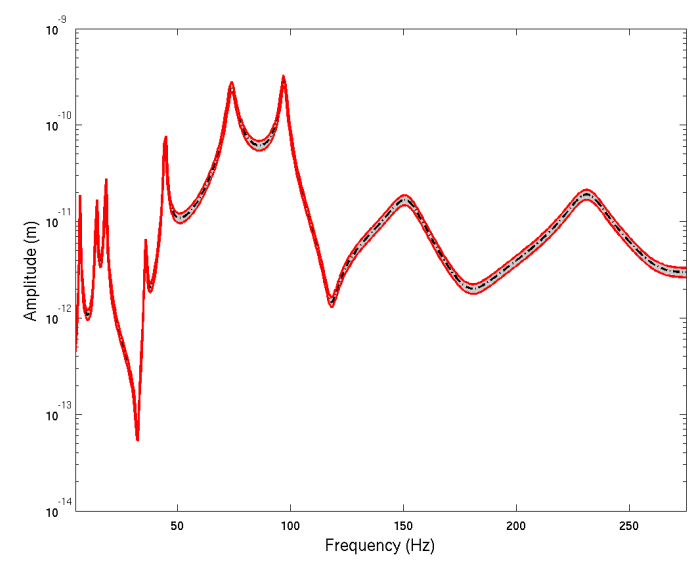

(e)

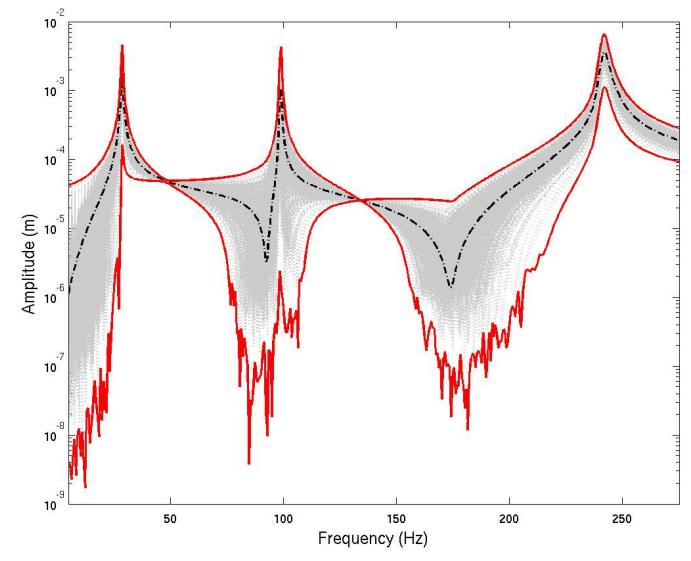

(b)

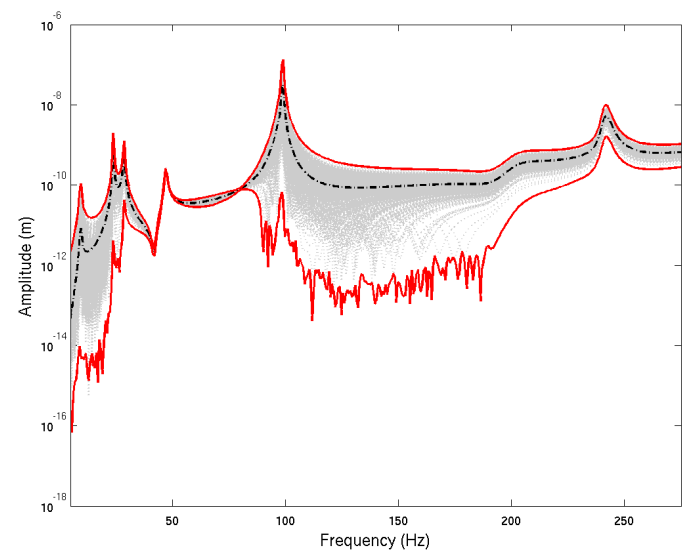

(d)

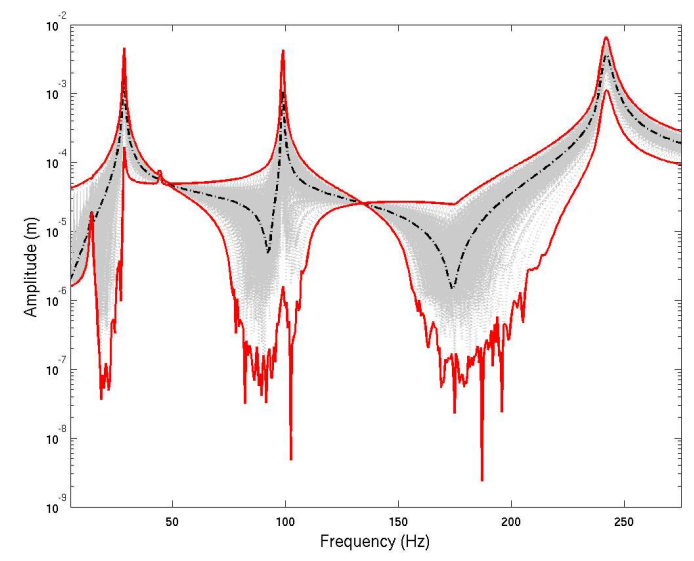

(f)

Figure 19: Frequency Response Functions (Case 7); Lower and upper envelopes (red solid line); MCS (gray dotted line); Deterministic response (black dotted dashed line): (a) static component; (b) $1 \times$ harmonic component; (c) $2 \times$ super-harmonic component; (d) $3 \times$ super-harmonic component; (e) $4 \times$ super-harmonic component; (f) global non-linear response. 

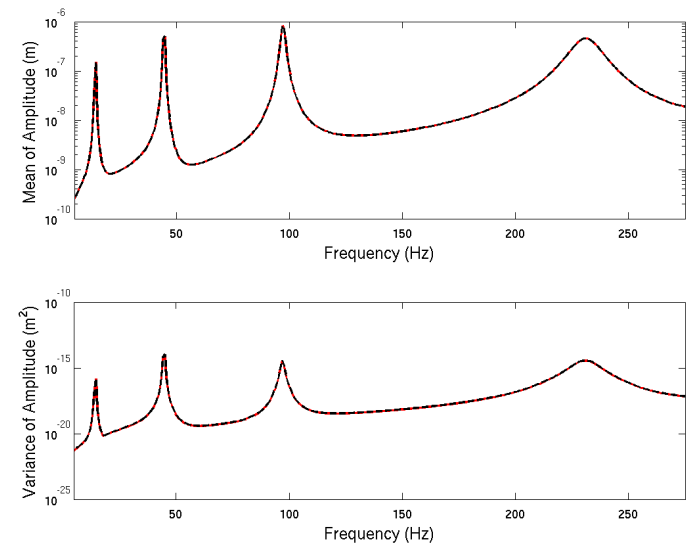

(a)
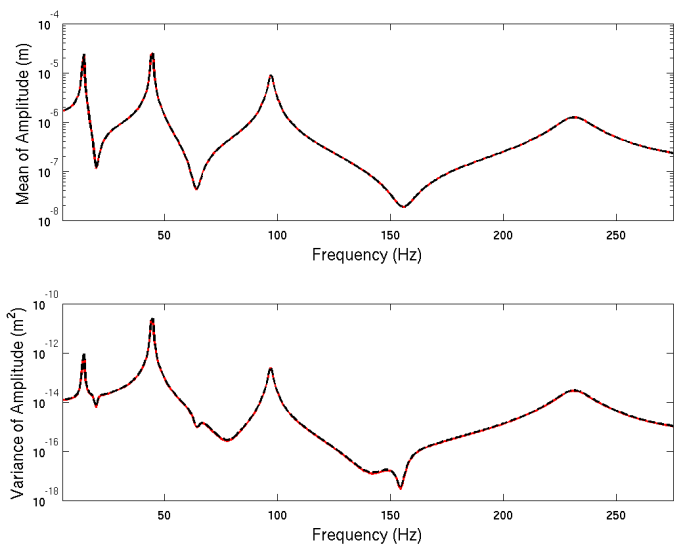

(c)
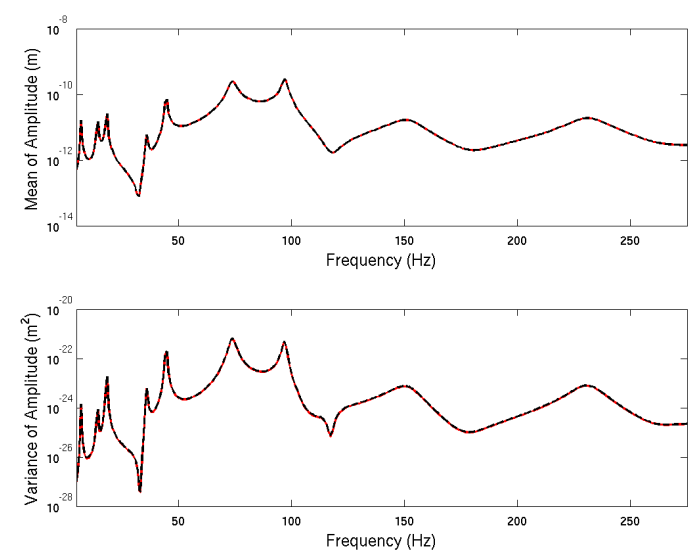

(e)
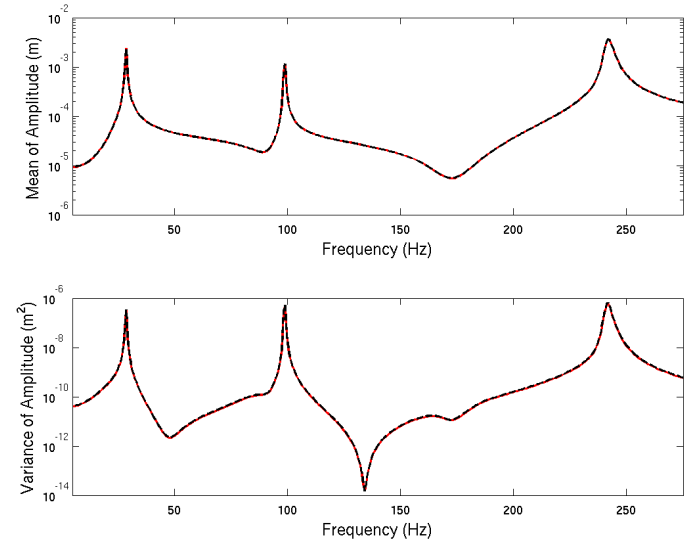

(b)
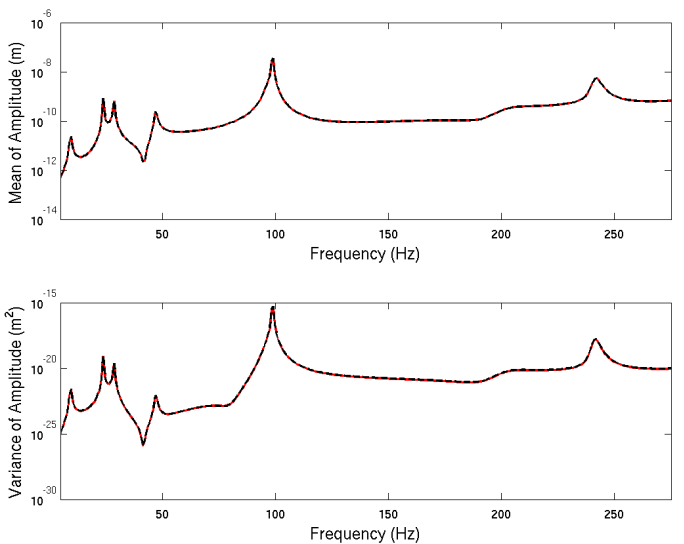

(d)
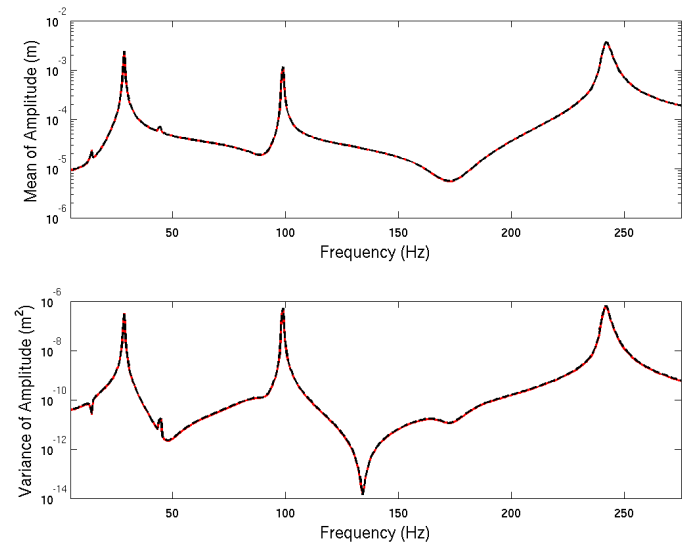

(f)

Figure 20: Frequency Response Functions (Case 8); Mean and Variance of the FRF with PCE (red solid line) and MCS (black dashed line): (a) static component; (b) $1 \times$ harmonic component; (c) $2 \times$ super-harmonic component; (d) $3 \times$ super-harmonic component; (e) $4 \times$ super-harmonic component; (f) global non-linear response. 


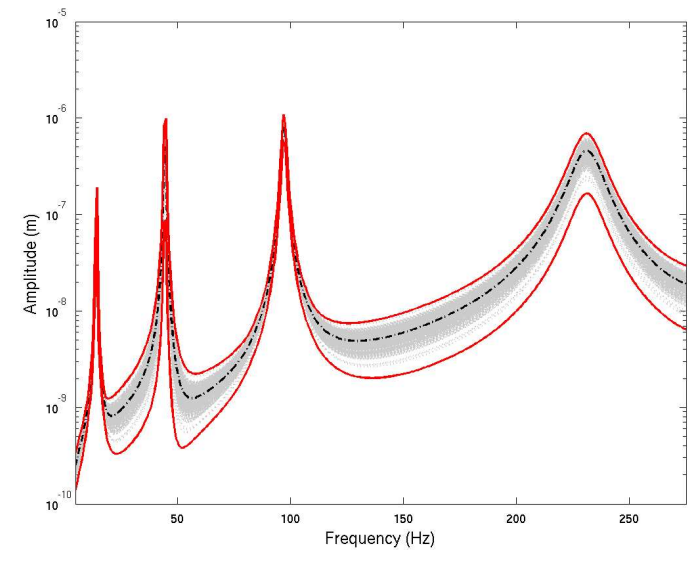

(a)

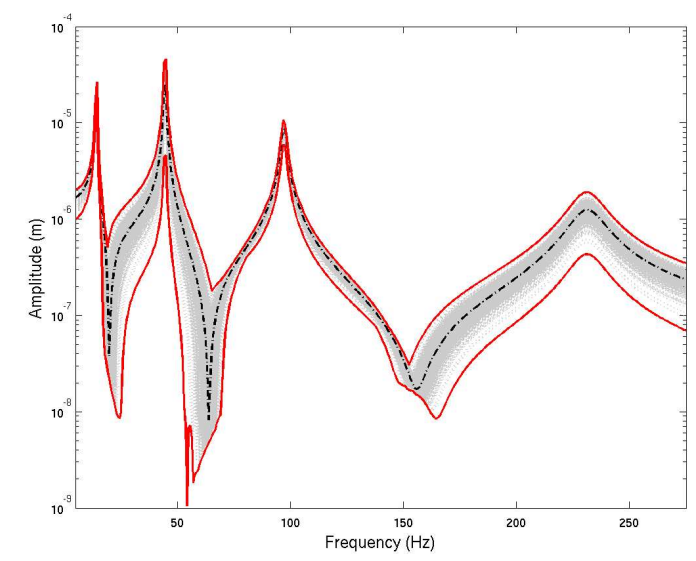

(c)

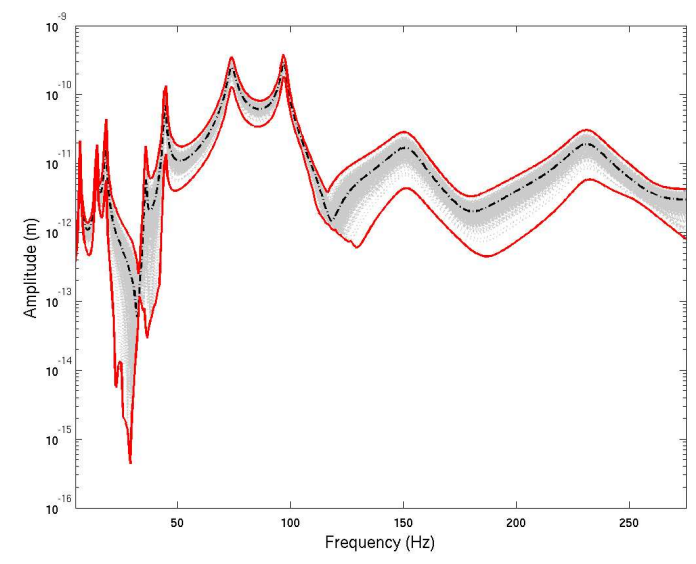

(e)

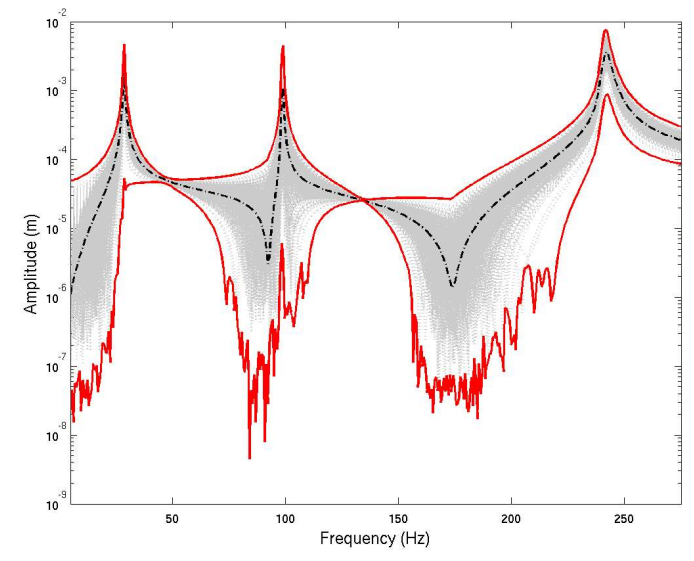

(b)

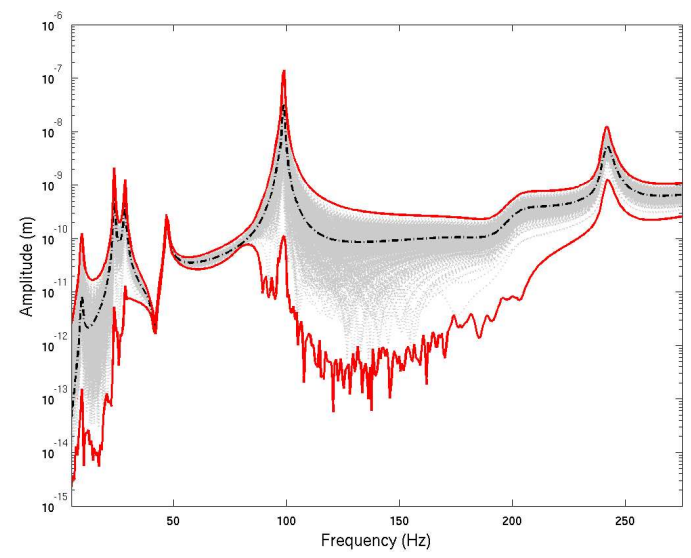

(d)

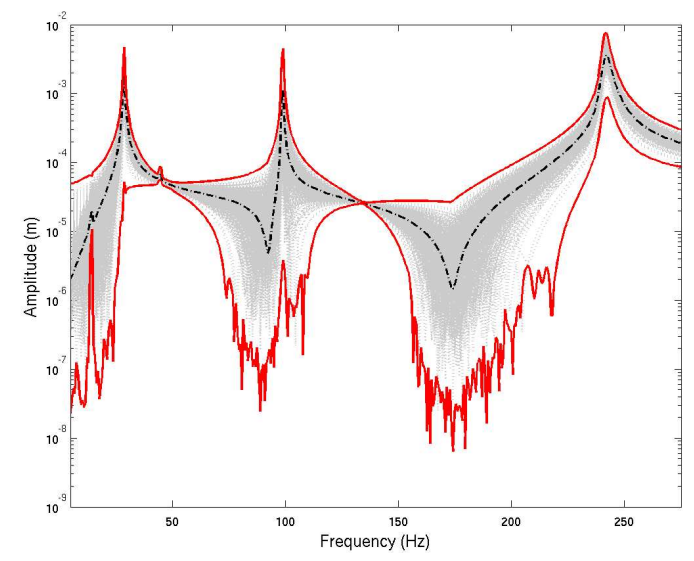

(f)

Figure 21: Frequency Response Functions (Case 8); Lower and upper envelopes (red solid line); MCS (gray dotted line); Deterministic response (black dotted dashed line): (a) static component; (b) $1 \times$ harmonic component; (c) $2 \times$ super-harmonic component; (d) $3 \times$ super-harmonic component; (e) $4 \times$ super-harmonic component; (f) global non-linear response. 
critical speeds respectively (at $28 \mathrm{~Hz}, 99 \mathrm{~Hz}$ and $243 \mathrm{~Hz}$ ). Figures 22(a) and 22(c) show orbits at the first and second $2 \times$ critical speeds respectively (at $14 \mathrm{~Hz}$ and $45 \mathrm{~Hz}$ ). For the $1 \times$ critical speeds simple loops are observed. For the $2 \times$ critical speeds double loops are observed, so it highlights the presence of $2 \times$ harmonic component. These results are in perfect agreement with the previous evolutions of the n-FRF illustrate on Figure 21.

\section{Conclusion}

This paper describes the use of the Polynomial Chaos Expansion approach coupled with the Harmonic Balance Method in a rotordynamics problem. The efficiency and robustness of the proposed method has been tested through various numerical results. The stochastic results are validated by comparison with the results obtained by Monte Carlo simulations.

These results show that variations of faults such as unbalance, bow and parallel misalignment may affect all the harmonic component of the rotor system, and variations of faults such as asymmetric section or angular misalignment can affect all the harmonic components.

\section{Acknowledgments}

The first author gratefully acknowledges the French Education Ministry which supports this research. The authors gratefully acknowledge the financial support of the French National Research Agency through the Young Researcher program ANR-07-JCJC-0059-01-CSD2. 


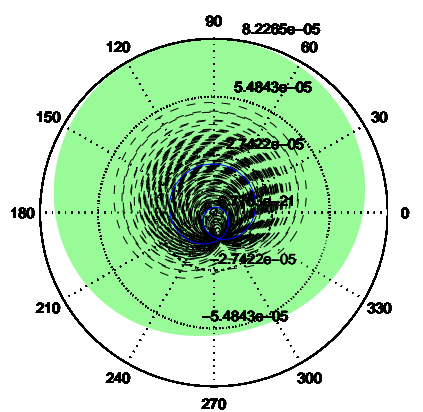

(a)

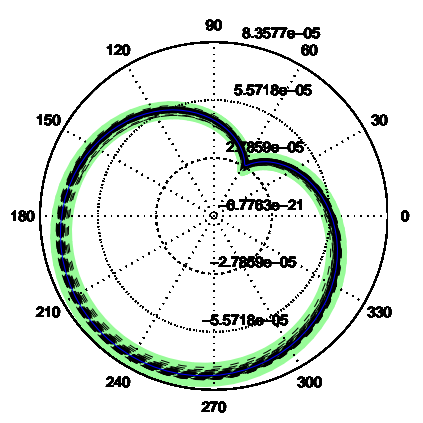

(c)

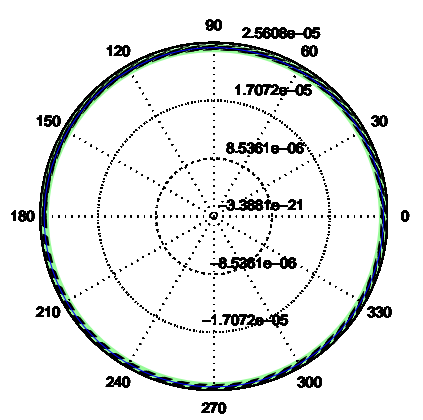

(e)

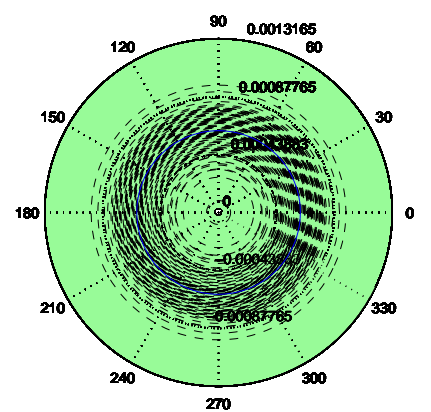

(b)

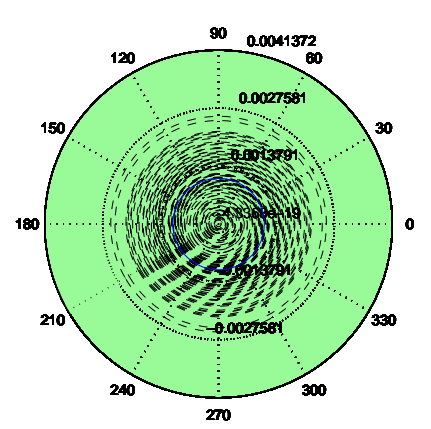

(d)

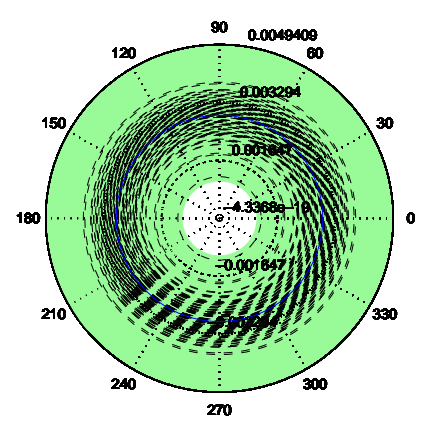

(f)

Figure 22: Orbits; PCE (green); MCS (black dashed line); Deterministic response (blue solid line): (a) $f=14 \mathrm{~Hz}$; (b) $f=28 \mathrm{~Hz}$; (c) $f=45 \mathrm{~Hz}$; (d) $f=99 \mathrm{~Hz}$; (e) $f=135 \mathrm{~Hz}$; (f) $f=243 \mathrm{~Hz}$. 


\section{References}

[1] S. Edwards, A.W. Lees, and M.I. Friswell. Fault diagnosis of rotating machinery. The Shock and Vibration Digest, 30(1):4-13, 1998.

[2] M. Xu and R.D. Marangoni. Vibration analysis of a Motor-Flexible Coupling-Rotor system subject to misalignment and unbalance, part i: Theoretical model and analysis. Journal of Sound and Vibration, 176(5):663-679, October 1994.

[3] M. Xu and R.D. Marangoni. Vibration analysis of a motor-flexible Coupling-Rotor system subject to misalignment and unbalance, part II: experimental validation. Journal of Sound and Vibration, 176(5):681-691, October 1994.

[4] K.M. Al-Hussain and I. Redmond. Dynamic response of two rotors connected by rigid mechanical coupling with parallel misalignment. Journal of Sound and Vibration, 249(3):483-498, January 2002.

[5] A.W. Lees. Some studies on misalignment in rigidly coupled flexible rotors. 7th IFToMMConference on Rotor Dynamics, Vienna, Austria, pages 25-28, 2006.

[6] A.W. Lees. Misalignment in rigidly coupled rotors. Journal of Sound and Vibration, 305(12):261-271, August 2007.

[7] J.E.T. Penny and M.I. Friswell. Simplified modelling of rotor cracks. ISMA 27, Leuven, Belgium, pages 607-615, 2002.

[8] J-J. Sinou and A.W. Lees. A non-linear study of a cracked rotor. European Journal of Mechanics - A/Solids, 26(1):152-170, January 2007.

[9] F. Oncescu, A.A. Lakis, and G. Ostiguy. Investigation of the stability and steady state response of asymmetric rotors, using finite element formulation. Journal of Sound and Vibration, 245(2):303-328, August 2001.

[10] M. Lalanne and G. Ferraris. Rotordynamics-Prediction in Engineering. John Wiley \& Sons, New York, 1990.

[11] P. Pennacchi, N. Bachschmid, A. Vania, G.A. Zanetta, and L. Gregori. Use of modal representation for the supporting structure in model-based fault identification of large rotating machinery: part 1-theoretical remarks. Mechanical Systems and Signal Processing, 20(3):662-681, April 2006.

[12] A.K. Darpe, K. Gupta, and A. Chawla. Dynamics of a bowed rotor with a transverse surface crack. Journal of Sound and Vibration, 296(4-5):888-907, October 2006.

[13] M. Richard and S. Malte. Model based fault identification in rotor systems by least squares fitting. International Journal of Rotating Machinery, 7(5):311-321, 2001.

[14] A. Nayfeh. Perturbation Methods. John Wiley and Sons, London, 1973. 
[15] H. Benaroya, and M. Rehak. Finite element methods in probabilistic structural analysis: A selective review. Applied Mechanics Reviews, 41(5):201-213, 1988.

[16] F. Yamazaki, M. Shinozuka, and G. Dasgupta. Neumann expansion for stochastic finite element analysis. Journal of Engineering Mechanics, ASCE, 114(8):1335-1354, 1988.

[17] R. Ghanem and P. Spanos. Stochastic Finite Elements: A Spectral Approach. Springer-Verlag, 1991.

[18] D.E. Bently, C.T. Hatch, and B. Grissom. Fundamentals of Rotating Machinery Diagnostics. Minden, Nev, USA: Bently Pressurized, Bearing Press, 2002.

[19] J-J. Sinou. Non-linear dynamics and contacts of an unbalanced flexible rotor supported on ball bearings. Mechanism and Machine Theory, 44(9):1713-1732, September 2009.

[20] M. Loève. Probability Theory, $4^{\text {th }}$ edition. Springer-Verlag, 1977. 